\title{
Verified Secure Compilation for Mixed-Sensitivity Concurrent Programs
}

\author{
Robert Sison* ${ }^{\dagger}$ and Toby Murray* \\ ${ }^{*}$ School of Computing and Information Systems, University of Melbourne, Australia \\ †CSIRO's Data61 and UNSW Sydney, Australia \\ (e-mail: [firstname].[lastname]@unimelb.edu.au)
}

\begin{abstract}
Proving only over source code that programs do not leak sensitive data leaves a gap between reasoning and reality that can only be filled by accounting for the behaviour of the compiler. Furthermore, software does not always have the luxury of limiting itself to single-threaded computation with resources statically dedicated to each user to ensure the confidentiality of their data. This results in mixed-sensitivity concurrent programs, which might reuse memory shared between their threads to hold data of different sensitivity levels at different times; for such programs, a compiler must preserve the value-dependent coordination of such mixed-sensitivity reuse despite the impact of concurrency.

Here we demonstrate, using Isabelle/HOL, that it is feasible to verify that a compiler preserves noninterference, the strictest kind of confidentiality property, for mixed-sensitivity concurrent programs. First, we present notions of refinement that preserve a concurrent value-dependent notion of noninterference that we have designed to support such programs. As proving noninterferencepreserving refinement can be considerably more complex than the standard refinements typically used to verify semantics-preserving compilation, our notions include a decomposition principle that separates the semantics-preservation from security-preservation concerns. Second, we demonstrate that these refinement notions are applicable to verified secure compilation, by exercising them on a single-pass compiler for mixed-sensitivity concurrent programs that synchronise using mutex locks, from a generic imperative language to a generic RISC-style assembly language. Finally, we execute our compiler on a nontrivial mixed-sensitivity concurrent program modelling a real-world use case, thus preserving its source-level noninterference properties down to an assembly-level model automatically. All results are formalised and proved in the Isabelle/HOL interactive proof assistant.

Our work paves the way for more fully featured compilers to offer verified secure compilation support to developers of multithreaded software that must handle data of multiple sensitivity levels.
\end{abstract}

\section{Introduction}

Here we show how to extend secure compilation support to programs that are designed to address two fundamental problems of scale: (1) the need to divide work in computer systems that handle information, and (2) the need to share scarce resources to be able to service every customer for whom that work is done. There will always be a program for which that sharing is not abstracted; that program's responsibility is to implement that sharing in such a way that it never allows the information of one customer to flow to another. In this paper, we prove formally that a compiler does not break that program's responsibility.

(C) CSIRO (ABN 41687119 230), Robert Sison, and Toby Murray 2019-2021; accepted manuscript to appear (excl. appendices) in CUP JFP Special Issue on Secure Compilation; licensed under CC BY-NC-ND 4.0 
It is well known that program translations of the kind carried out by compilers can easily break security properties like confidentiality (Kaufmann et al., 2016; Barthe et al., 2018). This is especially the case for mixed-sensitivity concurrent programs, which feature both:

- Concurrency of access to memory locations shared between different threads of execution. A compiler must preserve both (1) the synchronisation that coordinates threads' access to shared memory, and (2) the absence of any internal timing leaks, to prevent them from manifesting as storage leaks (Volpano \& Smith, 1998).

- Mixed-sensitivity reuse of shared memory to hold information of different sensitivity levels at different times. A compiler must preserve the program functionality that coordinates this reuse; this implies support for value-dependent classification policies, which allow the classification of a memory location to change dynamically depending on values held in other memory locations (Murray, 2015). Furthermore, it must do so accounting for the potential impact of concurrent access by other threads.

Although existing verified compilers for dialects of mainstream programming languages, like CompCert (Leroy, 2009) and CakeML (Kumar et al., 2014), have been proved to preserve program functionality (semantics) and some timing-sensitive forms of noninterference (Barthe et al. 2020), none are yet verified to preserve proofs of noninterference for mixed-sensitivity concurrent programs. Ideally such a compiler, applied to the threads of a proved-secure mixed-sensitivity concurrent program, would yield assembly code that, run concurrently, also composes into a secure mixed-sensitivity concurrent program.

To this end, here we present notions of concurrent value-dependent noninterferencepreserving refinement, which are compositional across the threads of mixed-sensitivity concurrent programs. In these notions, the usual square-shaped commuting diagram commonly used to depict (semantics-preserving) refinement (Figure 4a) has been replaced by a cube (Figure 3). This reflects that it preserves a 2-safety hyperproperty (Terauchi \& Aiken, 2005, Clarkson \& Schneider, 2010), which compares two executions rather than examining a single one. Our earlier work (Murray et al., 2016b) was the first to make this observation and to propose a general cube-shaped refinement property; however other work on verified secure compilation targeted towards noninterference preservation (Barthe et al., 2018, 2020) since made the same observation. As these cube-shaped properties are significantly more complicated to prove than standard notions of semantics-preserving refinement typical in verified compilation (Leroy, 2009, Kumar et al., 2014), we present a principle of decomposing the cube (Figure 3) into three separate obligations (Figure 4): the first of these is akin to semantics-preserving refinement, while the rest prevent the introduction of any termination- and timing-leaks. A simple comparison of proof effort for a refinement example (Figure 2) shows this approach can almost halve its complexity, and that it is applicable to proofs of refinement for programs with secret-dependent control flow-the example pads an if $h$ then ... else ... fi conditional with skips, so as not to introduce a timing leak of $h$.

We then go on to demonstrate that the decomposition principle we provide makes our notion of refinement a tractable target for verified secure compilation. Our compiler is an executable function in Isabelle/HOL that translates mixed-sensitivity concurrent programs that synchronise using mutex locks, from a generic imperative While language to a generic RISC-style assembly language. In particular, it supports the class of programs that avoid all 
implicitflows, where a secret determines the choice between two control flow paths with different observable effects, by disallowing any secret-dependent control flow-for example, disallowing if $h$ then ... else ... fi conditionals to prevent any timing leaks from $h$. This is a common approach against implicit flows, as it avoids any precise source-level reasoning about time. To preserve confidentiality for programs that take that approach, we instantiate the decomposition principle so that it enforces that our compiler does not introduce any new secret-dependent control flow. Furthermore, as part of satisfying the demands of our refinement notion, our compiler demonstrates a way of formalising and proving when it is safe for a compiler to perform optimisations in the presence of concurrency. To ensure that the contents of shared memory locations are preserved under compilation despite potential interference from other threads, our compiler tracks which shared memory locations are free from data races. It then makes use of this tracking to avoid redundant loads from "stable" (i.e. race-free) shared variables safely, that would otherwise be considered unsafe to omit.

Finally, to show that the compiler preserves noninterference for actual mixed-sensitivity concurrent programs, we execute it on a real-world use case: a model of the softwarecomponentised input-handling regime for the Cross Domain Desktop Compositor (Beaumont et al., 2016), a device that enforces information-flow control over input classified dynamically by a trusted user. We leave treatment on the design and application of perthread proof techniques establishing CVDNI for the successive versions of this model to other works (Murray et al., 2018; Sison, 2020), and here focus on its CVDNI-preserving compilation-expanding on Sison \& Murray (2019), the conference version of this paper. This yields the first proofs of noninterference for an assembly-level model of a nontrivial mixed-sensitivity concurrent program, demonstrating the power of verified secure compilation to preserve security properties of compiled code.

The structure of our paper is as follows. First, we present language-independent notions of noninterference and its refinement, designed for mixed-sensitivity concurrent programs (Section 2). Our attention then turns to preliminaries for our compiler: the main properties of interest of the source While language it compiles (Section 3), and of the target RISC language it produces (Section 4). Then, after presenting the details of our compiler and its verification Section 5), and the case study to which we apply it Section 6, we discuss the most closely related work in the area (Section 7), before concluding (Section 8).

We expand on the conference version of this paper (Sison \& Murray, 2019) as follows:

- Here we have adapted the noninterference properties to support assumptions on initial memory and extra security requirements; these will allow us to clarify exactly what our compiler is verified to preserve, and for which kinds of programs.

- We also in Section 2 present further preliminaries that will allow us to explain in greater detail the different ways to establish and use proofs about a verified compiler to obtain whole-system noninterference at the target-language level. These include:

1. The side conditions and theorem of compositionality for the noninterference properties. In Section 3, we then for the first time present the proof, whose details were elided from Sison \& Murray (2019), for a noncompositional "global" part of this side condition, which is necessary to obtain whole-system noninterference from per-thread noninterference both at source and target level. 
2. A whole-system refinement theorem, adapted to support assumptions on initial memory. This theorem was alluded to in Murray et al. (2016b) but, until now, has never been formally presented outside of the Isabelle/HOL theories. It gives us a means to prove preservation of whole-system noninterference by the compiler, without having to re-prove the noncompositional side condition at the target-language level.

- In Section 5, we then compare alternative methods of obtaining whole-system security at RISC level, that a developer would choose depending on whether all, or only some threads are compiled with our compiler. In contrast, Sison \& Murray (2019) stopped after presenting the application of refinement decomposition principle.

- In Section 6, the case study to which we apply the compiler is significantly expanded, being a 3-component version of the CDDC input-handling program-closer to a version presented in Murray et al. (2018) - as opposed to the 2-component version of Sison \& Murray (2019).

- Furthermore, we present substantially more details of our case study in Section 6. which were mostly elided from Sison \& Murray (2019). These include formal statements of both the source-level properties preserved and the target-level properties obtained, alongside explanations of all alternative methods for obtaining the latter from the former.

- Finally, every lemma and theorem we prove is presented with a proof sketch or explanation, which were largely absent from Sison \& Murray (2019). We also include here appendices with further details of our compiler (see Appendices $A, B$, and C.

\section{Noninterference and its refinement for mixed-sensitivity con- current programs}

To support mixed-sensitivity concurrent programs, we verify our compiler to preserve the concurrent value-dependent noninterference (CVDNI) notions of Murray et al. (2016b). In this section we present the definitions of CVDNI and its refinement, as we have adapted them from that work's Isabelle formalisation (Murray et al. 2016c a). In particular, the version of the theory we present here supports extra customisation of requirements beyond the prior work; we will need this to parameterise the theory with initial conditions needed for a compositionality property of our source language (Section 3), and our compiler's preservation of a ban on secret-dependent control flow (Section 5). Furthermore, it is simplified to the case where the shared memory is the same for both the original abstract and the refined concrete program-refinement adds no new shared variables. Later, we will instantiate this CVDNI theory to have our compiler's source and target languages (Sections 3, 44) respectively play the roles of the abstract and concrete programs' languages in the theory.

We begin by introducing with an illustrative example the challenges of verifying valuedependent noninterference in the presence of shared-variable concurrency (Section 2.1). Then we present the per-thread and whole-system noninterference properties themselves 


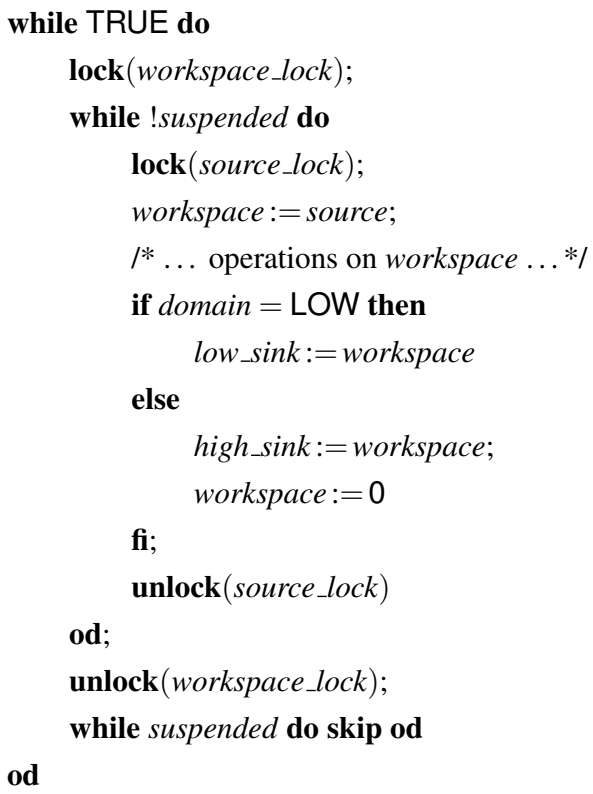

(a) Input processing worker thread program

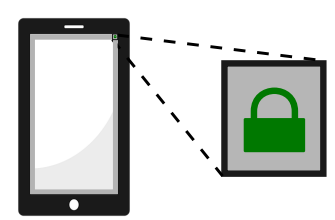

(b) The phone providing the High personality: domain $\neq \mathrm{LOW}$, and source is classified High to reflect that the user might type in secrets.

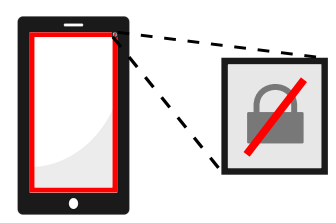

(c) The phone displaying visual indicators that it is providing the Low personality: domain $=$ LOW, and source is classified Low to reflect that we trust the user not to type in secrets.

Figure 1: Example: Touchscreen input processing for a dual-personality smartphone. Reproduced from Sison \& Murray (2019).

(Section 2.2, followed by the notion of per-thread refinement that preserves the perthread property between the two languages Section 2.3. As the cube-shapedness of noninterference-preserving refinement diagrams in general makes them difficult to apply directly to compiler verification, we present a decomposition principle (Section 2.4) that we will use to prove CVDNI-preserving refinement for our compiler. We then present requirements and a theorem for whole-system refinement by which we have that CVDNI-preserving refinement is compositional across the threads of the program being compiled, such that it yields the whole-system property at the target language level (Section 2.5).

\subsection{Illustrative example of a mixed-sensitivity concurrent program}

Consider the task of verifying a multithreaded system that manages the user interface (UI) for a dual-personality smartphone, a phone that provides clearly distinguished user contexts (personalities), typically for work versus leisure. Specifically, our task is to verify that it does not leak sensitive information intended only for one of those personalities, which we classify High (Figure 1b), to locations belonging to the other, which we classify Low (Figure 1c).

Here and generally throughout this paper, our attacker model is an entity that can read from the system's untrusted sinks: some subset of permanently Low-classified locations not subject to synchronisation. In our example, the untrusted sinks may include WLAN device registers in a hostile environment.

The smartphone's UI system consists of a number of threads running concurrently with a shared address space; we aim to verify that, as a whole, this system of threads satisfies the security requirement. However, to avoid a state space explosion that is exponential in the 
number of threads, we must do this compositionally: one thread at a time, then combining the results of these analyses.

We focus on a particular worker thread (Figure 1a), the one responsible for sending touchscreen input from the source variable to its intended destination.

The first challenge is that the destination depends on which personality the phone is currently providing, which is indicated by the value of domain. This is reflected by the classification of source being dependent on the value of domain: source is classified Low exactly when domain = LOW (where LOW is a designated constant), and is classified High otherwise. Due to this dependency, domain is known as a control variable of source.

The second challenge is the worker thread runs in a shared address space that might be accessed or modified by other threads, for various purposes. One of these threads may be responsible for maintaining that domain $=\mathrm{LOW}$ exactly when the phone indicates it is providing the Low personality (Figure 1c), so the user knows not to type in anything sensitive. Another thread may be responsible for assigning suspended:=TRUE when the user turns the phone's screen off, to make the worker stop processing touchscreen input. We may then wish for workspace to be usable by some other thread-for example, processing input from a fingerprint scanner-in such a way that it can assume that workspace no longer contains any sensitive values.

When we analyse one thread like this worker in terms of our compositional security property (Section 2.2), all the other threads in the system are trusted to do two things:

1. They follow a synchronisation discipline; in particular for this example, this is a mutual exclusion (mutex) locking discipline: If read- or write-access to a certain variable is governed by a lock, each thread may only access the variable in that manner if they hold that lock. Mutual exclusion then follows from the semantics of the locking primitives ensuring only one thread may hold a given lock at a time.

2. They themselves do not leak values from High-classified locations (we refer to such values themselves as High) to Low-classified locations that are read-accessible to other threads. Note that, here, it is our objective to prove that the thread we are analysing can be trusted in the same way.

Even under these assumptions, the concurrency gives rise to some tricky considerations.

First, it is important that no thread in the system (including the one under analysis) modifies any control variables carelessly. For example, writing domain $:=\mathrm{LOW}$ immediately after the worker reads a High value from source, will cause it to leak to low sink. To prevent this, the worker uses source_lock, granting it exclusive write-access to source and domain.

Furthermore, as noted above, we may want to ensure that a non-attacker-observable location is nevertheless cleared of any sensitive values before being used by another thread. In our example, we classify workspace Low for the analysis to enforce this when the worker is suspended, but as the worker sometimes uses it to process High values, it is important to know workspace is accessible only to the worker during that time. To ensure this, the worker uses workspace_lock, granting it exclusive read-and write-access to workspace. It is then responsible for clearing it of any High values by the time it releases that access. 


\subsection{Concurrent value-dependent notions of noninterference}

Having illustrated the challenges with an example, we now present the definitions of perthread and whole-system noninterference, the theorem by which the former composes into the latter, and the compositionality side conditions demanded by that theorem.

As proved for each thread, CVDNI is defined by Murray et al. (2016b) in terms of:

1. A binary strong low-bisimulation (modulo modes) relation $\mathscr{B}$ between program configurations, which serves as witness to CVDNI. In the style of other low-bisimulationbased noninterference definitions (Focardi et al., 1995; Sabelfeld \& Sands, 2000, Mantel et al. 2011) it requires the program configurations it relates to agree on their "low"-observable portions, and demands that lock-step execution preserves that correspondence. Furthermore, it is rely-guarantee-style concurrency aware, following Mantel et al. (2011), but modified to allow value-dependent classifications (Murray, 2015) for mixed-sensitivity reuse (see next point).

2. A classification function $\mathscr{L}$ that determines the "low"-observable portion of a program configuration, thus affecting $\mathscr{B}$ 's requirements. The innovation of $\mathscr{L}$, as parameterised first by Murray (2015) and then by Murray et al. (2016b) as reproduced here, is that $\mathscr{L}$ can depend on values in the program configuration itself, thus expressing dynamic and not just static classifications.

The theory is parameterised over the type of values $\mathrm{Val}$, a finite set of shared variables Var, and a deterministic evaluation step semantics $\rightsquigarrow$ between local configurations of a thread in a concurrent program. Each local configuration is a triple $\langle t p s, m d s, m e m\rangle$ :

- tps :: ThreadPrivate is the thread-private state, which the theory will consider to be permanently inaccessible to the attacker and not shared with the other threads. Note that, due to this inaccessibility, we allow the user of the theory to parameterise the type ThreadPrivate, and we do not impose any particular structure on it.

- $m d s:: M o d e \Rightarrow$ Var set is the (assume-guarantee) mode state, which is ghost state associating each of Mode $\triangleq\{$ AsmNoW, AsmNoRW, GuarNoW, GuarNoRW $\}$ with a set of shared variables. Intuitively, it identifies the set of variables for which the thread currently Assumes it possesses (or Guarantees it respects) exclusive permission to Write (or Read and Write), granted (or obligated) for those variables typically by some synchronisation scheme. This facilitates compositional, rely-guarantee-style reasoning about such access (Jones, 1981; Mantel et al., 2011).

For example, when our worker thread (of Figure 1a) holds source_lock, it assumes that no other threads write to source or its control variable domain (i.e. $\{$ source,domain $\} \subseteq m d s$ AsmNoW), otherwise it guarantees it does not write to them (GuarNoW). Similarly, when it holds workspace_lock it assumes that no other threads read or write to workspace (i.e. workspace $\in m d s$ AsmNoRW), and at all other times it makes the corresponding guarantee (GuarNoRW).

- mem :: Mem is shared memory considered potentially accessible to the attacker and other threads. To make what is accessible amenable to analysis, we impose the structure $\mathrm{Mem} \triangleq V a r \Rightarrow V a l$, a total map from shared variable names to values. 
The theory is then further parameterised by the value-dependent classification function $\mathscr{L}:: \mathrm{Mem} \Rightarrow \operatorname{Var} \Rightarrow\{$ High, Low $\}$, inducing a function $\mathscr{C}$ vars :: Var $\Rightarrow$ Var set that returns all the control variables of a given variable. In our worker thread example, $\mathscr{L}$ mem $x$ gives:

- High when $x$ is high_sink, meaning high_sink is classified High at all times.

- when $x$ is source: Low if mem domain $=\mathrm{LOW}$, and High otherwise.

- Low for all other variables $x$, meaning they are classified Low at all times.

The set $\mathscr{C}=\{y \mid \exists x, y \in \mathscr{C}$ vars $x\}$ is then defined to contain all control variables in the system. Thus in our worker thread example, $\mathscr{C}$ vars source $=\{$ domain $\}$ and $\mathscr{C}=\{$ domain $\}$.

With these parameters having been set, we can now define notions of observational equivalence - underpinning noninterference properties - that are value dependent.

The notion of observational equivalence of memories, used by the whole-system noninterference property to quantify over initial state pairs, is as follows: Variables that are value-dependently classified Low according to both memories are required to have the same value in both memories. Formally, as defined originally by Murray (2015):

Definition 2.1 (Low-equivalent memories).

$$
\text { mem }_{1}={ }^{\text {Low }} \text { mem }_{2} \triangleq \forall x . \mathscr{L} \text { mem }_{1} x=\text { Low } \longrightarrow \text { mem }_{1} x=\text { mem }_{2} x
$$

Note that the asymmetry of Definition 2.1 (also Definition 2.3 to follow) referring only to $m e m_{1}$ is resolved by requiring the classification function $\mathscr{L}$ to classify all control variables as Low statically - that is, Low always, regardless of the memory state (cf. our restriction Proposition 3.4 on the classification of state used to implement locks, later in Section 3.2.

To support compositionality for concurrent programs, however, the equivalence notion for the per-thread noninterference property is relaxed to be modulo modes in the style of Mantel et al. (2011): Here, Low-classified non-control variables $x \notin \mathscr{C}$ are only required to have the same value if they are assumed to be readable by other threads according to the mode state. (Control variables $x \in \mathscr{C}$ are excluded from that relaxation, and are always required to be equal.) Defined more formally, again as originally by Murray (2015), 1

Definition 2.2 (Readability of variable $x$, according to mode state $m d s$ ).

$$
\text { readable } m d s x \triangleq x \notin m d s \text { AsmNoRW }
$$

Definition 2.3 (Low-equivalence of memories, modulo the mode state $m d s$ ).

$$
\begin{aligned}
& \text { mem }_{1}={ }_{m d s}^{\text {Low }} \text { mem }_{2} \triangleq \\
& \forall x . x \in \mathscr{C} \vee \mathscr{L} \text { mem }_{1} x=\text { Low } \wedge \text { readable } m d s x \longrightarrow \text { mem }_{1} x=\text { mem }_{2} x
\end{aligned}
$$

Moreover, we will use notation $l c_{1}=\underset{\text { mds }}{\text { Low }} l c_{2}$ from Sison \& Murray (2019) to lift Definition 2.3 to local program configurations, asserting also that the local configurations $l c_{1}$ and $l c_{2}$ have the same assume-guarantee mode state. Additionally, we will use notation $l c_{1}={ }_{\text {mds }} l c_{2}$ to denote (only) that $l c_{1}$ and $l c_{2}$ have the same assume-guarantee mode state.

\footnotetext{
${ }^{1}$ Logical operator precedence here is just as in Isabelle/HOL — from most tightly to least: $\wedge, \vee, \longrightarrow$.
} 
Thus, intuitively, the user of the theory should model the permanent untrusted output sinks, of their whole concurrent program, as variables for which $\mathscr{L}$ always returns Low, ungoverned by any synchronisation scheme that the attacker cannot be trusted to follow. In our worker example program (of Figure 1a), low sink is untrusted permanently in this way, but workspace is untrusted only when unlocked.

We now have almost enough definitions to state the per-thread compositional security property. This property will assert the existence of a witness bisimulation relation $\mathscr{B}$ for every possible observationally equivalent pair of starting configurations. Specifically, this witness relation must be a strong low-bisimulation (modulo modes) (denoted by strong-low-bisim-mm $\mathscr{B}$ ), meaning that it must satisfy the following three conditions:

1. It must maintain observational indistinguishability by requiring that all configuration pairs it relates (i.e. $\left.\left(l c_{1}, l c_{2}\right) \in \mathscr{B}\right)$ that have the same mode state $\left(l c_{1}={ }_{\text {mds }} l c_{2}\right)$, are low-equivalent modulo modes $\left(l c_{1}={ }_{\mathrm{mds}}^{\text {Low }} l c_{2}\right)$.

2. Furthermore, it must be a bisimulation by being symmetric (denoted by sym $\mathscr{B}$ ) and progressing to itself: Any step taken by one of the configurations $\left(l c_{1} \rightsquigarrow l c_{1}^{\prime}\right)$ must be matched by some step taken by the configuration related to it $\left(l c_{2} \rightsquigarrow l c_{2}^{\prime}\right)$, so the destinations remain related (i.e. $\left.\left(l c_{1}^{\prime}, l c_{2}^{\prime}\right) \in \mathscr{B}\right)$ and modes-equal $\left(l c_{1}^{\prime}={ }_{\mathrm{mds}} l c_{2}^{\prime}\right)$.

3. Finally, it must be closed under globally consistent changes made to memory by other threads (denoted by cg-consistent $\mathscr{B}$ ) — that is, changes that preserve low-equivalence and are permitted by the current mode state $m d s$. Specifically, other threads are permitted to change either of variable $x$ 's value or its classification only when $x$ is considered writable by the current mode state (denoted by writable $m d s x$, Definition 2.5). This is the most crucial element of the per-thread CVDNI property itself that ensures its compositionality for concurrent programs.

These requirements are formalised by Definition 2.4, using Definitions 2.5 and 2.6 .

Definition 2.4 (Strong low bisimulation, modulo modes).

$$
\begin{aligned}
& \text { strong-low-bisim-mm } \mathscr{B} \triangleq \text { cg-consistent } \mathscr{B} \wedge \text { sym } \mathscr{B} \wedge \\
& \left(\forall l c_{1} l c_{2} \cdot\left(l c_{1}, l c_{2}\right) \in \mathscr{B} \wedge l c_{1}={ }_{\text {mds }} l c_{2} \longrightarrow\right. \\
& l c_{1}={ }_{\text {mds }}^{\text {Low }} l c_{2} \wedge \\
& \left.\left(\forall l c_{1}^{\prime} . l c_{1} \rightsquigarrow l c_{1}^{\prime} \longrightarrow\left(\exists l c_{2}^{\prime} . l c_{2} \rightsquigarrow l c_{2}^{\prime} \wedge l c_{1}^{\prime}=_{\text {mds }} l c_{2}^{\prime} \wedge\left(l c_{1}^{\prime}, l c_{2}^{\prime}\right) \in \mathscr{B}\right)\right)\right)
\end{aligned}
$$

Definition 2.5 (Writability of variable $x$, according to mode state $m d s$ ).

$$
\text { writable } m d s x \triangleq x \notin m d s \text { AsmNoW } \wedge x \notin m d s \text { AsmNoRW }
$$

Definition 2.6 (Closedness under globally consistent changes).

$$
\begin{aligned}
& \text { cg-consistent } \mathscr{B} \triangleq \forall t p s_{1} \text { mem }_{1} \text { tps }_{2} \text { mem }_{2} \text { mds. } \\
& \quad\left(\left\langle t p s_{1}, m d s, \text { mem }_{1}\right\rangle,\left\langle t p s_{2}, m d s, \text { mem }_{2}\right\rangle\right) \in \mathscr{B} \longrightarrow \\
& \left(\forall \text { mem }_{1}^{\prime} \text { mem }_{2}^{\prime} .\left(\forall x .\left(\text { mem }_{1} x \neq \text { mem }_{1}^{\prime} x \vee \text { mem }_{2} x \neq \text { mem }_{2}^{\prime} x \vee\right.\right.\right. \\
& \left.\left.\quad \mathscr{L} \text { mem }_{1} x \neq \mathscr{L} \text { mem }_{1}^{\prime} x\right) \longrightarrow \text { writable } m d s x\right) \wedge \text { mem }_{1}^{\prime}{ }^{\text {Low }}{ }_{m d s} \text { mem }_{2}^{\prime} \longrightarrow \\
& \left.\left(\left\langle t p s_{1}, m d s, \text { mem }_{1}^{\prime}\right\rangle,\left\langle t p s_{2}, m d s, \text { mem }_{2}^{\prime}\right\rangle\right) \in \mathscr{B}\right)
\end{aligned}
$$


Note that, to prevent unnecessary proof effort, strong-low-bisim-mm assumes instead of asserting the initial modes-equality $\left(l c_{1}={ }_{\mathrm{mds}} l c_{2}\right)$, as the security property that will use strong-low-bisim-mm will take responsibility for asserting it (to follow, in Definition 2.7).

We now present definitions of the CVDNI security properties that differ from those published in Murray et al. (2016b) and our conference paper Sison \& Murray (2019), in that they allow two additional forms of customisation as parameters to the theory, necessary for a fuller written presentation of the formal verification of our compiler:

1. Initialisation requirements for the system, in the form of a predicate over shared memory called INIT.

The per-thread and whole-system security properties are relaxed such that they only quantify over initial shared memories that obey this predicate.

2. Extra requirements to be imposed on top of strong low-bisimulation modulo modes, in the form of a predicate over bisimulation relations called EXTRA.

The per-thread security property is strengthened to impose these additional requirements on any candidate security witness.

When dropped from each of the names of the properties "com-secure" and "sys-secure" soon to be introduced, INIT and EXTRA default to ( $\lambda_{-}$. True); in that case, the definitions of those properties will then simplify to their original versions as presented in Murray et al. (2016b); Sison \& Murray (2019).

The per-thread security property is then as follows:

Definition 2.7 (Per-thread compositional security, with INIT, EXTRA requirements).

$$
\begin{aligned}
& \text { com-secure }{ }_{\text {INIT }}^{\text {EXTRA }}(\text { tps }, m d s) \triangleq \forall \text { mem }_{1} \text { mem }_{2} . \\
& \text { mem }_{1}={ }_{\text {mds }}^{\text {Low }} \text { mem }_{2} \wedge \text { INIT mem } 1 \wedge \text { INIT mem } \text { m }_{2} \longrightarrow \\
& (\exists \mathscr{B} \text {. strong-low-bisim-mm } \mathscr{B} \wedge \text { EXTRA } \mathscr{B} \wedge \\
& \left.\left(\left\langle t p s, \text { mds } \text { mem }_{1}\right\rangle,\left\langle\text { tps }, \text { mds }, \text { mem }_{2}\right\rangle\right) \in \mathscr{B}\right)
\end{aligned}
$$

We have proved in Isabelle/HOL that the compositionality theorem of Murray et al. (2016b) holds regardless of the INIT, EXTRA chosen - in short, the INIT condition relaxes the goal sufficiently to relax each of its assumptions the same way, and the EXTRA requirement only strengthens its assumptions. Subject to some "sound mode use" side conditions (to be discussed soon), it gives us that the parallel composition $\mathrm{cms}::$ (ThreadPrivate $\times$ (Mode $\Rightarrow$ Var set)) list of com-secure program threads will itself be a concurrent program that enforces "sys-secure", a system-wide value-dependent noninterference property. Here, the set operator returns the set of all the elements in a given list:

Theorem 2.8 (Compositionality of com-secure ${ }_{I N I T}^{\text {EXTRA }}$ ).

$$
\begin{gathered}
\forall(t p s, m d s) \in \text { set } \mathrm{cms} . \text { com-secure } \text { INIT }_{\text {INI }}^{\text {EXT }}(\mathrm{tps}, \mathrm{mds}) \\
\forall \text { mem. INIT mem } \longrightarrow \text { sound-mode-use }(\mathrm{cms}, \text { mem })
\end{gathered}
$$


We first introduce the elements of this whole-system property "sys-secure", before defining it formally (to follow, in Definition 2.9).

From all low-equivalent pairs of initial memories that both satisfy the INIT conditions, this whole-system property "sys-secure" asserts a form of low-equality between all global configuration pairs that are reachable via evaluation $-\rightarrow \rightarrow_{\text {sched }}$ to the same fixed schedule sched, for all such finite lists sched giving an order of steps of execution from each thread:

$$
\begin{aligned}
& g c-\rightarrow_{[]} g c^{\prime} \triangleq\left(g c=g c^{\prime}\right) \\
& g c \rightarrow_{n . n s} g c^{\prime} \triangleq\left(\exists g c^{\prime \prime} . g c \rightsquigarrow_{n} g c^{\prime \prime} \wedge g c^{\prime \prime} \rightarrow_{n s} g c^{\prime}\right)
\end{aligned}
$$

Here [] is an empty list, ..- is the cons operator, and $\rightsquigarrow_{n}$ means the $n$th thread in the global configuration takes one step.

In always comparing pairs of runs executing against the same schedule, the property models the class of schedulers whose decisions never depend on any secrets. Consequently, this excludes schedulers that are specialised, in the manner of Barthe et al. (2007a), to actively monitor the sensitivity level of each thread's control flow, so as to intervene and avoid interleaving it with others when it has become dependent on secrets. Instead, the CVDNI theory puts the onus on the developer of the program to prove that any branching on secret conditionals does not lead to timing-sensitive flows of the secret as discernible via low-classified sinks accessible to other threads in the system. Note that, as CVDNI prohibits mode state from ever becoming secret dependent, it will implicitly prohibit any leaks into parts of memory with which the mode state is directly associated-in Section 3, we will need to prohibit leaks into the memory we use to implement mutex locks, for this reason.

The special form of low-equality applied by the whole-system property is one that is modified from Definition 2.1, so that it only requires each Low-classified non-control variable $x \notin \mathscr{C}$ to be of equal value in both global configurations if the mode states of all threads consider $x$ to be readable (Definition 2.2). Furthermore, the property ensures that paired global configurations continue to agree on the number of threads in the system, and on the mode states for all threads, written $c m s_{1}^{\prime}=$ all-mds $c m s_{2}^{\prime} \triangleq\left(\right.$ map mds $c m s_{1}^{\prime}=$ map mds $\left.c m s_{2}^{\prime}\right)$, where the syntax "map mds $c m s$ " denotes the mapped projection that extracts a list of mode states from a list $c m s$ of ThreadPrivate $\times($ Mode $\Rightarrow$ Var set) pairs. Finally, we will use syntax $\mathrm{cms}[i]$ to denote the $i$ th element in list $\mathrm{cms}$.

This whole-system noninterference property, written formally, is then as follows:

Definition 2.9 (Whole-system value-dependent security, with INIT requirements).

$$
\begin{aligned}
& \text { sys-secure }_{I N I T} \mathrm{cms} \triangleq \forall \text { mem }_{1} \text { mem }_{2} \text {. } \\
& \text { INIT mem } 1 \wedge \text { INIT mem } \text { }_{2} \wedge \text { mem }_{1}={ }^{\text {Low }} \text { mem }_{2} \longrightarrow \\
& \left(\forall \text { sched } \mathrm{cms}_{1}^{\prime} \mathrm{mem}_{1}^{\prime} \text {. }\left(\mathrm{cms}, \mathrm{mem}_{1}\right) \rightarrow \rightarrow_{\text {sched }}\left(\mathrm{cms}_{1}^{\prime}, \mathrm{mem}_{1}^{\prime}\right) \longrightarrow\right. \\
& \left(\exists c m s_{2}^{\prime} \text { mem }_{2}^{\prime} .\left(c m s, \text { mem }_{2}\right) \rightarrow \rightarrow_{\text {sched }}\left(c m s_{2}^{\prime}, \text { mem }_{2}^{\prime}\right)\right) \wedge \\
& \left(\forall c m s_{2}^{\prime} \text { mem }_{2}^{\prime} \text {. }\left(\mathrm{cms}, \mathrm{mem}_{2}\right)-\rightarrow_{\text {sched }}\left(\mathrm{cms}_{2}^{\prime}, \mathrm{mem}_{2}^{\prime}\right) \longrightarrow\right. \\
& \text { length } c m s_{1}^{\prime}=\text { length } c m s_{2}^{\prime} \wedge c m s_{1}^{\prime}={ }_{\text {all-mds }} c m s_{2}^{\prime} \wedge \\
& \left(\forall x . x \in \mathscr{C} \vee \mathscr{L} \mathrm{mem}_{1}^{\prime} x=\text { Low } \wedge\right. \\
& \left.\left.\left.\left(\forall i<\text { length } c m s_{1}^{\prime} \text {. readable } c m s_{1}^{\prime}[i] x\right) \longrightarrow \operatorname{mem}_{1}^{\prime} x=\operatorname{mem}_{2}^{\prime} x\right)\right)\right)
\end{aligned}
$$


Finally, we must note that the use of assume-guarantee reasoning to obtain the compositionality of the per-thread property in the style of Mantel et al. (2011) gives rise to requirements justifying the soundness of that reasoning; requirements that we will prove our compiler to preserve. For CVDNI, these are summed up by the "sound-mode-use" side condition of Theorem 2.8, which consists of a "local" and a "global" part:

Definition 2.10 (Sound mode use side-condition).

$$
\begin{aligned}
& \text { sound-mode-use }(\mathrm{cms}, \mathrm{mem}) \triangleq \\
& \quad(\forall \mathrm{cm} \in \text { set } \mathrm{cms} \text {. local-mode-compliance }(\mathrm{cm}, \mathrm{mem})) \wedge \\
& \text { global-modes-compatibility }(\mathrm{cms}, \mathrm{mem})
\end{aligned}
$$

First, all threads must each obey a local mode compliance requirement. This says that for all reachable local configurations of the program, at no point will the thread violate any of its own guarantees not to access a particular location in the shared state, which implies also not accessing any of its control variables. We leave precise definitions for "reachable-lcs" and "doesnt-read-(or-modify)" to this paper's Isabelle/HOL supplement material: ${ }^{2}$ but mention here that it is the doesnt-read-(or-modify) assertions that enforce that any guarantees not to access some variable $x$ will effectively apply also to all of $x$ 's control variables:

Definition 2.11 (Local mode compliance).

local-mode-compliance $l c \triangleq$

$\forall c$ mds mem. $\langle c, m d s, m e m\rangle \in$ reachable-lcs $l c \longrightarrow$ respects-own-guarantees $(c, m d s)$

where

$$
\begin{aligned}
& \text { respects-own-guarantees }(c, m d s) \triangleq \\
& \qquad \begin{array}{l}
(\forall x .(x \in m d s \text { GuarNoRW } \longrightarrow \text { doesnt-read-or-modify } c x) \wedge \\
(x \in m d s \text { GuarNoW } \longrightarrow \text { doesnt-modify } c x))
\end{array}
\end{aligned}
$$

Then, all threads must together obey a global modes compatibility requirement. This requirement says that the threads' mode states in all reachable global configurations of the concurrent program (the "reachable-mds-lists") are compatible - that is, if any one thread assumes a particular location will not be accessed for writing or reading, then all other threads must be guaranteeing not to access that location for the same purpose:

Definition 2.12 (Global modes compatibility).

global-modes-compatibility $g c \triangleq \forall m d s s \in$ reachable-mds-lists $g c$. compatible-modes $m d s s$ where

$$
\begin{aligned}
& \text { reachable-mds-lists } g c \triangleq \\
& \left\{m d s s \mid \exists c m s^{\prime} \text { mem }^{\prime} \text { sched. } g c-\rightarrow_{\text {sched }}\left(\mathrm{cms}^{\prime}, \text { mem }^{\prime}\right) \wedge \text { map mds } c m s^{\prime}=m d s s\right\} \\
& \text { compatible-modes } m d s s \triangleq \forall i x . i<\text { length } m d s s \longrightarrow \\
& (x \in m d s s[i] \text { AsmNoRW } \longrightarrow \\
& \quad(\forall j<\text { length } m d s s . j \neq i \longrightarrow x \in m d s s[j] \text { GuarNoRW })) \wedge \\
& (x \in m d s s[i] \text { AsmNoW } \longrightarrow \\
& \quad(\forall j<\text { length } m d s s . j \neq i \longrightarrow x \in m d s s[j] \text { GuarNoW }))
\end{aligned}
$$

\footnotetext{
${ }^{2}$ The Isabelle/HOL theories are available at http://covern.org/jfpsc.html
} 


\section{if $h \neq 0$ then \\ $x:=y$ \\ else \\ $x:=y+z$ \\ fi}

(a) Abstract if-conditional.

Relation $\mathscr{R}$ pairs configurations of this program with configurations of the program in Figure $2 b$ that are of the same-shaded region.

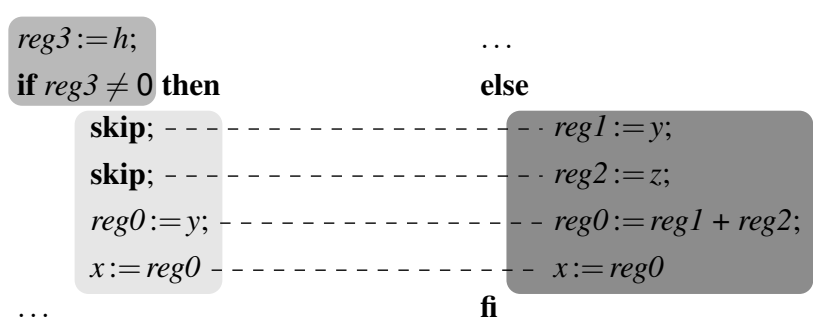

(b) Concrete if-conditional. Relation $\mathscr{I}$ pairs configurations of this program as shown by the dashed lines.

Figure 2: Excerpts from a CVDNI-preserving refinement example with secret-dependent control flow: $h$ contains a secret, $y$ and $z$ contain zero, and $x$ is an untrusted sink. Reproduced from Sison \& Murray (2019) — the example is originally from Murray et al. (2016b).

Note that this global modes compatibility requirement is not compositional; consequently, instead of obliging the program developer to prove it for the source programs to be fed to our compiler, we will prove it as an invariant maintained by the execution semantics of our source language - particularly, by its synchronisation primitives (see Section 3 ).

For more details and precise presentations of all the definitions we have adapted from Murray et al. (2016b c) to enable the compiler verification work described in this paper, please refer to the Isabelle/HOL formalisation in our supplement material.

\subsection{Cube-shaped refinement for preserving noninterference}

Proof of CVDNI-preserving refinement (also security-preserving or secure refinement), for a single-threaded program that will be run as a thread of a concurrent program, requires the user of the theory to nominate two binary relations (both illustrated by Figure 2):

1. A refinement relation $\mathscr{R}$ relating local configurations of the abstract program to local configurations of the concrete program: Abstract must simulate concrete, in a sense typical of much other work on program refinement, including compiler verification.

2. A concrete coupling invariant $\mathscr{I}$ that allows us to use $\mathscr{B}$ and $\mathscr{R}$ to build a new strong low-bisimulation (modulo modes) for the concrete program, by discarding pairs of local configurations after the refinement that should not be reached in the same number of evaluation steps. It thereby witnesses that any changes a refinement (or compiler) might make to the execution time do not introduce any timing channels.

The essence of the proof technique is to require that a number of conditions-analogous to those for strong-low-bisim-mm (Definition 2.4) - be imposed on the nominated $\mathscr{R}$ and $\mathscr{I}$, in relation to a given witness relation $\mathscr{B}$ establishing com-secure (Definition 2.7) for the abstract program. The definitions to follow are adapted from Murray et al. (2016b) Section $\mathrm{V}$, as we presented in Sison \& Murray (2019) — for better readability, a simplified version in which no new shared variables are added by the refinement. Consequently, we use the notation $=\underset{m d s}{m e m}$ to denote that two local configurations have equal mode state and memory, regardless of whether relating configurations of the same or differing languages. 

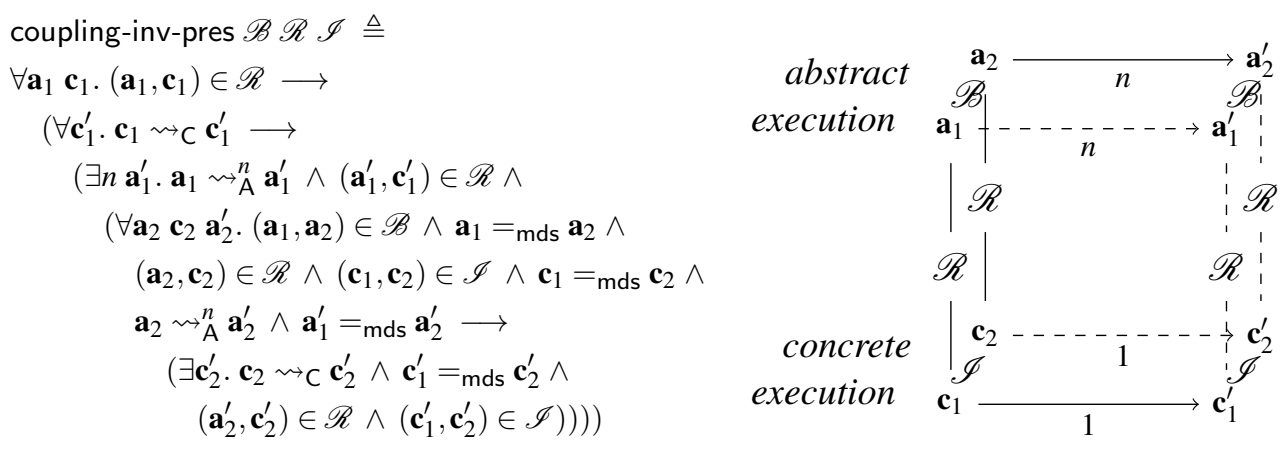

Figure 3: Definition and graphical depiction of refinement preservation obligation for secure-refinement (Definition 2.15). Reproduced from Sison \& Murray (2019) - the definition is a simplified restatement of its original formalisation in Murray et al. (2016b).

Regarding the maintenance of modes equivalence and observational equivalence across the relation, the restrictions on refinement are tighter than those that were applied to strong-low-bisim-mm, in that $\mathscr{R}$ is required to preserve the shared memory in its entirety:

Definition 2.13 (Preservation of modes and memory).

$$
\text { preserves-modes-mem } \mathscr{R} \triangleq \forall l c_{A} l c_{C} \cdot\left(l c_{A}, l c_{C}\right) \in \mathscr{R} \longrightarrow l c_{A}={ }_{\mathrm{mds}}^{\mathrm{mem}} l c_{C}
$$

Regarding the closedness under changes by other threads that ensures compositionality for concurrency, on $\mathscr{I}$ we again impose cg-consistent (Definition 2.6) from Section 2.2. However, in the case of $\mathscr{R}$, we instead impose "closed-others", a simplification of cg-consistent that considers only environmental actions that affect the memories on both sides of the relation identically. Furthermore, closed-others ensures equality of all shared variables, not just those judged observable. Defined formally:

Definition 2.14 (Closedness of refinements under changes by others).

$$
\begin{aligned}
& \text { closed-others } \mathscr{R} \triangleq \forall t p s_{A} t p s_{C} \text { mds mem mem }{ }^{\prime} . \\
& \left.\quad\left(\left\langle t p s_{A}, m d s, m e m\right\rangle_{\mathrm{A}},\left\langle t p s_{C}, m d s, m e m\right\rangle_{\mathrm{C}}\right) \in \mathscr{R}\right) \wedge \\
& \quad\left(\forall x .\left(\text { mem } x \neq m e m^{\prime} x \vee \mathscr{L} \text { mem } x \neq \mathscr{L} m e m^{\prime} x\right) \longrightarrow \text { writable } m d s x\right) \longrightarrow \\
& \left.\quad\left(\left\langle t p s_{A}, m d s, \text { mem }^{\prime}\right\rangle_{\mathrm{A}},\left\langle t p s_{C}, m d s, \text { mem }^{\prime}\right\rangle_{\mathrm{C}}\right) \in \mathscr{R}\right)
\end{aligned}
$$

The final major-and hardest-requirement for confidentiality preservation is to prove $\mathscr{R}$ and $\mathscr{I}$ closed simultaneously under the pairwise executions of the concrete and abstract programs, using a cube-shaped "refinement and coupling invariant preservation" diagram (coupling-inv-pres, depicted in Figure 3), whose edges are configuration pairs in $\mathscr{B}, \mathscr{R}$, and $\mathscr{I}$. (Reducing its difficulty is the focus of the decomposition principle in Section 2.4.)

All that then remains is for the nominated concrete coupling invariant $\mathscr{I}$ to be symmetric (sym $\mathscr{I}$ ), and the predicate secure-refinement puts together all the requirements:

Definition 2.15 (Requirements for confidentiality-preserving secure refinement). secure-refinement $\mathscr{B} \mathscr{R} \mathscr{I} \triangleq$ preserves-modes-mem $\mathscr{R} \wedge$ closed-others $\mathscr{R} \wedge$ cg-consistent $\mathscr{I} \wedge$ sym $\mathscr{I} \wedge$ coupling-inv-pres $\mathscr{B} \mathscr{R} \mathscr{I}$ 
The soundness theorem for confidentiality-preserving refinement by Murray et al. (2016b) then gives us that, under these conditions, the concrete relation " $\mathscr{B}$ C of $\mathscr{B} \mathscr{R} \mathscr{I}$ ", derived from a witness strong-low-bisim-mm relation $\mathscr{B}$, refinement relation $\mathscr{R}$, and coupling invariant $\mathscr{I}$, is itself a witness strong-low-bisim-mm for the concrete program. For readability, from here onwards we will use $\mathbf{a}_{1}, \mathbf{c}_{1}, \ldots$ instead of $l c_{1 A}, l c_{1 C}, \ldots$ for local configuration variables when comparing abstract and concrete executions simultaneously:

Definition 2.16 (Concrete bisimulation relation derived from $\mathscr{B}, \mathscr{R}$ and $\mathscr{I}$ ).

$$
\begin{aligned}
& \mathscr{B}_{\mathrm{C}} \text { of } \mathscr{B} \mathscr{R} \mathscr{I} \triangleq\left\{\left(\mathbf{c}_{1}, \mathbf{c}_{2}\right) \mid\right. \exists \mathbf{a}_{1} \mathbf{a}_{2} .\left(\mathbf{a}_{1}, \mathbf{c}_{1}\right) \in \mathscr{R} \wedge\left(\mathbf{a}_{2}, \mathbf{c}_{2}\right) \in \mathscr{R} \wedge \\
&\left.\left(\mathbf{a}_{1}, \mathbf{a}_{2}\right) \in \mathscr{B} \wedge \mathbf{c}_{1}={ }_{\text {mds }}^{\text {Low }} \mathbf{c}_{2} \wedge\left(\mathbf{c}_{1}, \mathbf{c}_{2}\right) \in \mathscr{I}\right\}
\end{aligned}
$$

Theorem 2.17 (Preservation of strong-low-bisim-mm by secure-refinement).

$$
\frac{\text { strong-low-bisim-mm } \mathscr{B} \quad \text { secure-refinement } \mathscr{B} \mathscr{R} \mathscr{I}}{\text { strong-low-bisim-mm }(\mathscr{B} \text { cof } \mathscr{B} \mathscr{R} \mathscr{I})}
$$

\subsection{Decomposition principle and its impact on refinement proofs}

We now present, as we first did in Sison \& Murray (2019), an alternative way to prove secure-refinement Definition 2.15) that obviates the need to use the cube-shaped, two-sided refinement obligation (depicted by Figure 3, by decomposing its concerns into:

1. Proving $\mathscr{R}$ closed using a square-shaped simulation diagram (depicted by Figure 4a akin to the backward simulations commonly used to prove semantics-preserving refinement by compilers (e.g. for CompCert (Leroy, 2009)), and

2. Security-focused proof obligations (depicted by Figures $4 b$, 4c), separable from the square-shaped simulation, that prevent the introduction of timing leaks, termination leaks, and secret-dependent differences in assume-guarantee mode state.

The decomposition requires the verifier to nominate a new parameter, called abs-steps or the pacing function. Its role is to dictate the pace of the square-shaped simulation by specifying the number of abstract steps that ought to be taken for one concrete step, as depicted by Figure 4a. Deferring the security-focused side conditions ("decomp-refinement-safe") to afterwards, the decomposition principle is then defined formally as follows:

Definition 2.18 (Decomposition principle for secure-refinement).

$$
\begin{aligned}
& \text { secure-refinement-decomp } \mathscr{B} \mathscr{R} \mathscr{I} \text { abs-steps } \triangleq \\
& \text { preserves-modes-mem } \mathscr{R} \wedge \text { closed-others } \mathscr{R} \wedge \text { cg-consistent } \mathscr{I} \wedge \text { sym } \mathscr{I} \wedge \\
& \text { decomp-refinement-safe } \mathscr{B} \mathscr{R} \mathscr{I} \text { abs-steps } \wedge(\forall \mathbf{a} \mathbf{c} .(\mathbf{a}, \mathbf{c}) \in \mathscr{R} \longrightarrow \\
& \left.\quad\left(\forall \mathbf{c}^{\prime} . \mathbf{c} \rightsquigarrow \mathrm{C} \mathbf{c}^{\prime} \longrightarrow\left(\exists \mathbf{a}^{\prime} . \mathbf{a}{ }_{\mathrm{A}}^{(\text {abs-steps } \mathbf{a} \mathbf{c})} \mathbf{a}^{\prime} \wedge\left(\mathbf{a}^{\prime}, \mathbf{c}^{\prime}\right) \in \mathscr{R}\right)\right)\right)
\end{aligned}
$$

The aforementioned side conditions on all refinement parameters, depicted by Figures $4 \mathrm{~b}, 4 \mathrm{c}$, are then defined formally under the predicate decomp-refinement-safe as follows: 


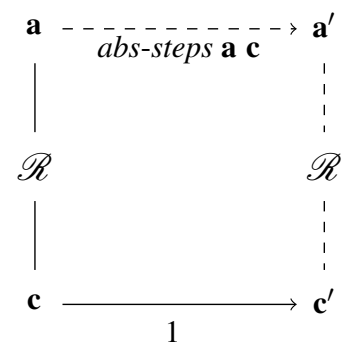

(a) Refinement preservation for relation $\mathscr{R}$ under program execution paced by abs-steps. (Part of Definition 2.18)

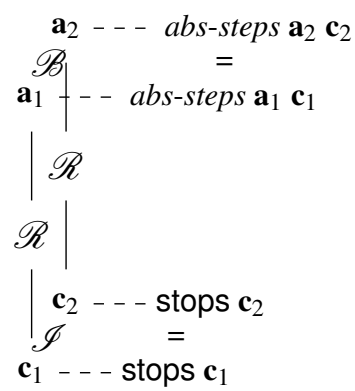

(b) Consistency of pacing and stopping behaviour, to prevent timing and termination leaks. (Part of Definition 2.19.)

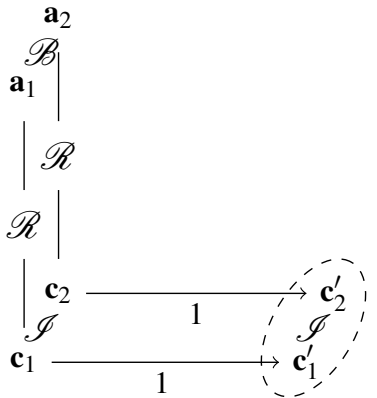

(c) Closedness of the coupling invariant relation $\mathscr{I}$ under lockstep program execution. (Part of Definition 2.19)

Figure 4: Graphical depictions of decomposed refinement preservation obligations. Reproduced from Sison \& Murray (2019).

Definition 2.19 (Security-focused side conditions for decomposition principle).

$$
\begin{aligned}
& \text { decomp-refinement-safe } \mathscr{B} \mathscr{R} \mathscr{I} \text { abs-steps } \triangleq \forall \mathbf{a}_{1} \mathbf{a}_{2} \mathbf{c}_{1} \mathbf{c}_{2} \cdot\left(\mathbf{a}_{1}, \mathbf{a}_{2}\right) \in \mathscr{B} \wedge \\
& \mathbf{a}_{1}={ }_{\text {mds }} \mathbf{a}_{2} \wedge\left(\mathbf{a}_{1}, \mathbf{c}_{1}\right) \in \mathscr{R} \wedge\left(\mathbf{a}_{2}, \mathbf{c}_{2}\right) \in \mathscr{R} \wedge\left(\mathbf{c}_{1}, \mathbf{c}_{2}\right) \in \mathscr{I} \wedge \mathbf{c}_{1}={ }_{\text {mds }} \mathbf{c}_{2} \\
& \longrightarrow \text { stops } \mathbf{c}_{1}=\text { stops } \mathbf{c}_{2} \wedge \text { abs-steps } \mathbf{a}_{1} \mathbf{c}_{1}=\text { abs-steps } \mathbf{a}_{2} \mathbf{c}_{2} \wedge \\
& \quad\left(\forall \mathbf{c}_{1}^{\prime} \mathbf{c}_{2}^{\prime} \cdot \mathbf{c}_{1} \rightsquigarrow \mathrm{C} \mathbf{c}_{1}^{\prime} \wedge \mathbf{c}_{2} \rightsquigarrow \mathrm{C} \mathbf{c}_{2}^{\prime} \longrightarrow\left(\mathbf{c}_{1}^{\prime}, \mathbf{c}_{2}^{\prime}\right) \in \mathscr{I} \wedge \mathbf{c}_{1}^{\prime}={ }_{\text {mds }} \mathbf{c}_{2}^{\prime}\right)
\end{aligned}
$$

The intuitive meanings of the side conditions in Definition 2.19 are:

- stops $\mathbf{c}_{1}=$ stops $\mathbf{c}_{2}$ ensures that the refinement has not introduced any termination leaks, by asserting consistent stopping behaviour for $\mathscr{I}$-related concrete program configurations, which we know to be observationally indistinguishable.

- abs-steps $\mathbf{a}_{1} \mathbf{c}_{1}=$ abs-steps $\mathbf{a}_{2} \mathbf{c}_{2}$ ensures that the refinement has not introduced any timing leaks, by asserting consistency of the pace of the refinement for $\mathscr{R}$-related program configurations, which we again know to be observationally indistinguishable.

- The final $\forall$-quantified clause asserts $\mathscr{I}$ 's suitability as a coupling invariant, in that it must remain closed under lockstep evaluation of the concrete program configurations it relates. Furthermore it must maintain mode state equality with each lockstep evaluation, which ensures that the refinement has not introduced any inconsistencies in the memory access assumptions and guarantees needed for the concurrent compositionality of the property.

Note that the $\mathscr{B}$ - and $\mathscr{R}$-edges in Figure $4 \mathrm{c}$ may capture useful facts about a particular program verification technique and compiler (respectively), so their availability as assumptions is intended to reduce greatly the effort needed to specify a coupling invariant $\mathscr{I}$ and prove it satisfies the condition.

Assuming the fulfilment of all the decomposed requirements, we obtain that they are a sound method for establishing secure refinement of the per-thread confidentiality property, as desired: 
Theorem 2.20 (Soundness of the decomposition principle).

secure-refinement-decomp $\mathscr{B} \mathscr{R} \mathscr{I}$ abs-steps $\Longrightarrow$ secure-refinement $\mathscr{B} \mathscr{R} \mathscr{I}$

Proof. The only obligation for secure-refinement (Definition 2.15) not obtained immediately from secure-refinement-decomp (Definition 2.18) is the cube-shaped coupling-inv-pres (Figure 3). We discharge this as follows:

The front face of the cube is just ordinary square-shaped refinement preservation (depicted in Figure 4a, given to us by secure-refinement-decomp: that a single concrete step from $\mathbf{c}_{1}$ is simulated by $n$ abstract steps from $\mathbf{a}_{1}$, where $n$ is given by abs-steps.

We are then obliged to prove a simulation in the other direction (the back face of the cube), that $n$ abstract steps from all configurations $\mathbf{a}_{2}$ related by $\mathscr{B}$ to $\mathbf{a}_{1}$ are simulated by some concrete step from $\mathbf{c}_{2}$ related by $\mathscr{R}$ to $\mathbf{a}_{2}$ and by $\mathscr{I}$ to $\mathbf{c}_{1}$.

Here, we lean on the determinism of the abstract program's evaluation semantics (required by the theory) to flip the direction of simulation, knowing that $n$ abstract steps from $\mathbf{a}_{2}$, simulating a single concrete step from $\mathbf{c}_{2}$, could only be the very same $n$ abstract steps from $\mathbf{a}_{2}$ that we were required to consider. This allows us to obtain that simulation by using, once again, the square-shaped refinement preservation (Figure 4a) given to us by secure-refinement-decomp.

Consistency of refinement pacing and stopping behaviour (depicted in Figure 4b) given by decomp-refinement-safe (Definition 2.19) then respectively ensure that $n$ (via abs-steps) is the correct number of abstract steps to consider, and that there will indeed be a concrete step from $\mathbf{c}_{2}$ to drive the matching simulation step.

Finally, the remainder of decomp-refinement-safe (depicted in Figure 4c) discharges the requirement of closedness and modes-equality maintenance of $\mathscr{I}$ under lockstep execution, demanded by the bottom face of the cube.

To demonstrate how the decomposition principle reduces proof complexity and effort, we returned to the example program refinement discussed in Section V-E of Murray et al. (2016b) and proved in its Isabelle formalisation (Murray et al., 2016a), an excerpt of which is shown in Figure 2. The abstract program (9 imperative commands) branches on a sensitive value, and executes a single atomic expression assignment in each branch. Its refinement (to 16 commands) models expansion of the expressions into multiple steps, resolving a timing disparity between the two branches by padding with skip.

We use proof size as a proxy for proof effort, since the former is known to be strongly linearly correlated with the latter (Staples et al., 2014). Formalised in Isabelle/HOL as EgHighBranchRevC. thy (Murray et al., 2016a), the proof line count for that theory stood at about $4.6 \mathrm{~K}$ lines of definitions and proof, of which approx. $3.6 \mathrm{~K}$ line were proofs. Adapting the proof instead to use the decomposition principle (secure-refinement-decomp, Definition 2.18), the proof line count drops from $3.6 \mathrm{~K}$ to approx. $2 \mathrm{~K}$, a $44 \%$ reduction. Regarding definition changes, the new proof makes less than 10 lines of adaptations to a coupling invariant and pacing function used by the old proof, and adds about 30 lines worth of new helper definitions, for use with the decomposition principle. The rest of the theory and its external dependencies remain in common between the two versions.

As would be expected, the bulk of the deletions are from the full cube-shaped refinement diagram proof (Figure 3) of secure-refinement (Definition 2.15) for the refinement relation. The surviving parts of that proof just become the square-shaped refinement diagram proof 
(Figure 4a) of the decomposition principle (Definition 2.18), without much modification. The deletions are replaced by newly added proofs of the decomposition principle's more security-focused side conditions (Definition 2.19, depicted by Figures 4b, 4c).

\subsection{Compositional whole-system secure refinement}

We now present the whole-system refinement theorem from Murray et al. (2016b a), which we adapt here to support the specification of INIT requirements (as in com-secure INIT , Definition 2.77, and simplify to the case of refinements that add no shared variables.

The main usefulness of this theorem is that, beyond demanding secure-refinement (Definition 2.15, which dealt with the preservation of per-thread security as witnessed by a strong-low-bisim-mm (Definition 2.4), it deals additionally with the preservation of the sound-mode-use side conditions (Definition 2.10) that will be demanded by the compositionality theorem for CVDNI (Theorem 2.8) at the target language level.

Notably, although it imposes the requirement for the refinement to preserve the "local" part of sound-mode-use Definition 2.11, it automatically preserves the non-compositional "global" part of this side condition (Definition 2.12) as a consequence of the requirements imposed by the per-thread secure refinements. Thus, our source-level proof of the global condition (see Section 3 will be sufficient, and there will be no need for us to prove anything extra about our compiler for it to preserve that to the target-language level.

We now present the requirements and theorem for whole-system refinement formally.

First, in addition to the per-thread refinement notion secure-refinement (Definition 2.15) that we addressed in Sections 2.3 and 2.4, our whole-system refinement theorem will require that the refinement relation $\mathscr{R}$ established by the compiler additionally preserves the compositional local mode compliance property for each thread. Here, "respects-own-guarantees" is from Definition 2.11:

Definition 2.21 (Refinement $\mathscr{R}$ preserves local mode compliance).

$$
\begin{gathered}
\text { preserves-local-compliance } \mathscr{R} \triangleq \forall t p s_{A} m d s_{A} m e m_{A} t p s_{C} m d s_{C} m e m_{C} . \\
\text { respects-own-guarantees }\left(t p s_{A}, m d s_{A}\right) \wedge \\
\left(\left\langle t p s_{A}, m d s_{A}, m e m_{A}\right\rangle_{\mathrm{A}},\left\langle t p s_{C}, m d s_{C}, m e m_{C}\right\rangle_{C}\right) \in \mathscr{R} \longrightarrow \\
\text { respects-own-guarantees } \left.\left(t p s_{C}, m d s_{C}\right)\right)
\end{gathered}
$$

We define a new "compositional refinement" predicate to capture all per-thread requirements that will be demanded by our compositional whole-system refinement theorem. This bundles together preserves-local-compliance and secure-refinement so as to preserve the strong-low-bisim-mm relations (Definition 2.4) that witness noninterference for each thread of the abstract program. Alongside all these requirements just described, it also requires the concrete coupling invariant $\mathscr{I}$ to cover all possible initial memory pairs that are low-equal modulo modes (Definition 2.3) and satisfy the $I N I T_{C}$ conditions that will parameterise the target language-level CVDNI property: 
Definition 2.22 (Requirements for compositional whole-system refinement).

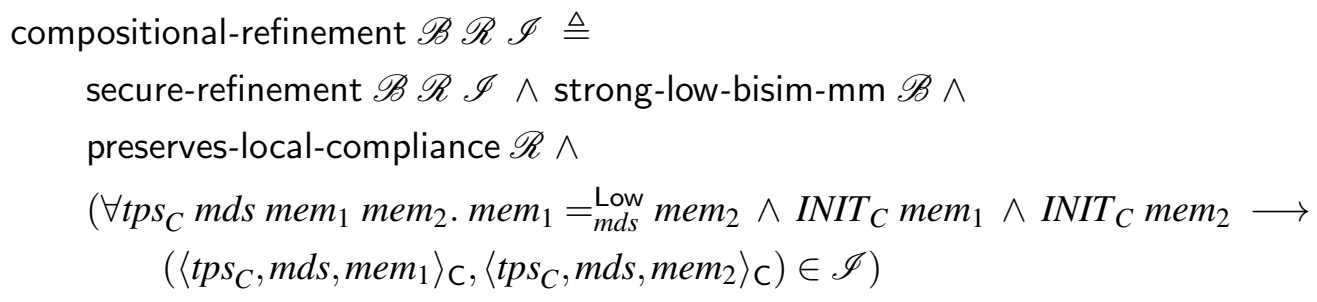

With these requirements, we prove using Isabelle/HOL that a whole-system refinement theorem, proved originally by Murray et al. (2016ba), can be adapted to support the specification of INIT requirements on initial memory at both abstract- and concrete-level. (As with Theorem 2.8, the relaxation of the goal by $I N I T_{C}$ is enough to permit the relaxations of its assumptions by $I N I T_{C}, I N I T_{A}$.) First we will state the theorem, then we will explain it, line-by-line. This theorem proves that abstract-level sound-mode-use (including its global part) by a system of secure mixed-sensitivity concurrent program threads (i.e. list $c m s_{A}$, as witnessed by bisimulations $\mathscr{B}$ s for each thread) is sufficient for a set of per-thread secure refinements (in terms of the lists $\mathscr{B} \mathrm{s}, \mathscr{R} \mathrm{s}, \mathscr{I}$ s of bisimulation, refinement, and concrete coupling invariant relations for each thread, respectively) to yield a concrete-level secure concurrent program (i.e. list $\mathrm{cms}_{C}$ that satisfies sys-secure, Definition 2.9p:

Theorem 2.23 (Whole-system compositionality of per-thread secure refinement).

$$
\begin{aligned}
& \left(\forall \text { mem. INIT } \text { I }_{\text {mem }} \longrightarrow \text { INIT }_{A} \text { mem }\right) \wedge \\
& \left(\forall \text { mem. INIT } T_{A} \text { mem } \longrightarrow \text { sound-mode-use }\left(\mathrm{cms}_{A}, \mathrm{mem}\right)\right) \wedge \\
& \text { length } c m s_{A}=\text { length } \mathscr{B} \mathrm{s}=\text { length } \mathscr{R} \mathrm{s}=\text { length } \mathscr{I} \mathrm{s}=\text { length } c m s_{C} \wedge \\
& \left(\forall i<\text { length } \mathrm{cms}_{C}\right. \text {. } \\
& \text { compositional-refinement } \mathscr{B} \mathrm{s}[i] \mathscr{R} \mathrm{s}[i] \mathscr{I} \mathrm{s}[i] \wedge \\
& \left(\forall \text { mem. INIT } T_{C} \text { mem } \longrightarrow\left(\left(\mathrm{cms}_{A}[i], \text { mem }\right),\left(\mathrm{cms}_{C}[i], \text { mem }\right)\right) \in \mathscr{R} \mathrm{s}[i]\right) \wedge \\
& \left(\forall \text { mem }_{1} \text { mem }_{2} . \text { INIT }_{A} \text { mem }_{1} \wedge \operatorname{INIT}_{A} \text { mem }_{2} \wedge \text { mem }_{1}={ }_{\left(\text {snd } \mathrm{cms}_{C}[i]\right)}^{\text {Low }} \text { mem }_{2} \longrightarrow\right. \\
& \left.\left.\left(\left(\mathrm{cms}_{A}[i], \text { mem }_{1}\right),\left(\mathrm{cms}_{A}[i], \text { mem }_{2}\right)\right) \in \mathscr{B} \mathrm{s}[i]\right)\right) \\
& \text { sys-secure }{ }_{I N I T_{C}} \mathrm{Cms}_{C}
\end{aligned}
$$

The premises of this theorem can be understood as follows:

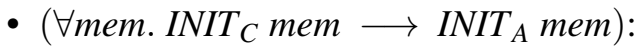

The concrete-level "INIT $C$ " initial condition must be no weaker than the abstract-level "INIT $T_{A}$ " one.

- $\left(\forall\right.$ mem. INIT $T_{A}$ mem $\longrightarrow$ sound-mode-use $\left.\left(\mathrm{cms}_{A}, \mathrm{mem}\right)\right)$ :

For the abstract program, sound-mode-use (Definition 2.10) must hold for all possible initial memories.

- The lists of initial thread-private and mode states at abstract and concrete level (resp. $\mathrm{cms}_{A}, \mathrm{cms}_{C}$ ), and lists of bisimulation, refinement, and concrete coupling invariant relations (resp. $\mathscr{B} \mathrm{s}, \mathscr{R} \mathrm{s}, \mathscr{I} \mathrm{s}$ ) must all be for the same number of threads. 
- Then, for all threads $i$ in the system:

- The relations $\mathscr{B}, \mathscr{R}, \mathscr{I}$ for thread $i$ must meet the requirements for "compositional whole-system refinement" (Definition 2.22).

- The refinement relation $\mathscr{R}$ for thread $i$ must hold initially, i.e. cover its initial thread-private and mode states at concrete and abstract level (resp. $c m s_{C}, c m s_{A}$ ), for all initial memories that satisfy the concrete $I N I T_{C}$ requirement.

- The abstract bisimulation relation $\mathscr{B}$ for thread $i$ must hold initially, i.e. must relate its initial thread-private and mode state to itself, for all pairs of memories that are low-equal modulo that mode state, and that both satisfy the abstract $I N I T_{A}$ requirement.

Given all these assumptions, Theorem 2.23 yields a whole-system noninterference property sys-secure ${ }_{I N I T_{C}}$ for the resulting concurrent program (with the list of initial threadprivate and mode states $c m s_{C}$ ) that assumes that the initial memory satisfies $I N I T_{C}$.

\section{Source language: While with mutex locks}

In this section, we give a focused presentation of our compiler's source language, centered on its properties that enable the composition of per-thread proofs of CVDNI-preserving refinement to the compiler's target RISC language. Our Isabelle/HOL supplement provides full formalisations of its semantics, and of instances of all per-thread proof techniques for proving CVDNI itself (developed for While by Sison (2020); Murray et al. (2016b c)).

While with mutex locks (hereafter While) is a generic imperative language with support for conditional looping, consisting of the commands $\mathrm{cmd}$ over arithmetic expressions exp:

$$
\begin{aligned}
\exp ::= & n|v| \exp \oplus \exp \\
\operatorname{cmd}::= & \text { skip }|c m d ; c m d| \text { if } \exp \text { then } c m d \text { else } c m d \text { fi } \mid \\
& \text { while } \exp \text { do } c m d \text { od }|v:=\exp | \text { stop } \mid \\
& \operatorname{lock}(k) \mid \mathbf{u n l o c k}(k)
\end{aligned}
$$

The language is parameterised over shared program-variable identifiers $v::$ Var, shared lock-variable identifiers $k::$ Lock, constant values $n::$ Val, and binary arithmetic operators $\oplus:: \mathrm{Val} \Rightarrow \mathrm{Val} \Rightarrow \mathrm{Val}$ that each have a big-step evaluation semantics; these induce a bigstep evaluation semantics for $\exp$ as a whole. The commands $c m d$ then have a small-step operational semantics, wherein skip and variable assignment $v:=\exp$ execute in one step to stop (which itself does not step to anything); conditional branch if steps to the appropriate cmd depending on whether its expression evaluates to zero; and conditional loop while steps to an if-conditional between either (1) the loop body sequenced with a repetition of the while command, or (2) stop. Finally the sequential command $c_{1} ; c_{2}$ executes to $c_{2}$ when $c_{1}$ executes to stop, and to $c_{1}^{\prime} ; c_{2}$ ( $c_{1}^{\prime}$ being $c_{1}$ 's destination) otherwise. Of these aforementioned commands, only variable assignments can modify the shared memory (program-variables only), and none can directly modify the mode state or lock-variables.

We will give special focus to the addition to the While language of the mutex synchronisation primitives $\operatorname{lock}(k)$ and $\operatorname{unlock}(k)$, which are the sole means of modifying lock variables and mode state. These replace both the ad-hoc mode annotations and the await $(v)$ 
synchronisation primitive that were previously offered for While by Murray et al. (2016b). After briefly noting here how While instantiates the underlying theory from Section 2 , we will present these new primitives' operational semantics, which depends on the program developer supplying details of the locking discipline as a parameter (Section 3.1) subject to some restrictions (Section 3.2). We will then prove that global-modes-compatibility (Definition 2.12) is invariant for systems of While programs running concurrently (Section 3.3), subject to some initial conditions (Section 3.4). Discharging this once-off noncompositional proof obligation is crucial in enabling both composition of per-thread noninterference properties (using Theorem 2.8), and compositional whole-system secure refinement of noninterference down to RISC by our compiler (using Theorem 2.23).

While instantiates the concurrent value-dependent noninterference theory described in Section 2.2. This instantiation assumes that the underlying concurrent execution model (e.g. operating system, scheduler) for the While language prevents threads from seeing each others' current program location. Thus the While program command $c:: c m d$ being executed (understood as the current program location) is modelled as the thread-private state of the local configuration triple: $\langle c, m d s, m e m\rangle_{\mathrm{w}}$. (The subscript ${ }_{\mathrm{w}}$ distinguishes While program triples from RISC ones, which are subscripted $r$.)

To ease formalisation of $\operatorname{lock}(k)$ and $\operatorname{unlock}(k)$, we instantiate the shared mem :: Mem type as a total mapping from a sum type to values Val. This sum type, with constructors Lock, Var, distinguishes lock-variable identifiers $k::$ Lock (which can only be read or written by the lock primitives) from program-variable identifiers $v:: \operatorname{Var}$ (which can be read or written by the rest of the commands). In Isabelle/HOL's datatype notation, this is:

$$
M e m \triangleq(\text { Lock Lock } \mid \text { Var Var }) \Rightarrow \text { Val }
$$

For readability, we will elide this distinction between Lock and Var-or applications of their constructors Lock and Var-from the presentation whenever clear from the context.

\subsection{Locking discipline and its semantics}

The program developer provides the details of the program's locking discipline in the form of a lock interpretation parameter lock-interp :: Lock $\Rightarrow$ (Var set $\times$ Var set $)$, which gives for each lock the two non-overlapping sets of program-variables over which acquiring the lock grants exclusive permission to write, (resp.) read and write. For readability, this presentation will elide lock-interp from the arguments of definitions, and use the notation varsNoW, varsNoRW :: Lock $\Rightarrow$ Var set to refer to its fst and snd projection.

Alongside encoding the mutex primitives' usual effect on control flow-most crucially, $\operatorname{lock}(k)$ should refuse to proceed meaningfully if the lock $k$ is already held-we will now specify for them an evaluation semantics that furthermore encodes the permissions implied by the locking discipline, as assumptions and guarantees expressed in the mode state. This semantics assumes that, initially, no locks are held, and all threads are making guarantees not to access the variables they govern (conditions we will define formally in Section 3.4).

The following two helpers specify how acquiring (resp. releasing) a lock affects the mode state under a given lock interpretation lock-interp. When a thread acquires a lock it gains more assumptions, and makes fewer guarantees about the region of memory concerned: 
Definition 3.1 (Impact on mode state $m d s$ of acquiring lock $k$ ).

$$
\begin{aligned}
& m d s \oplus k \triangleq \lambda m \text {. case } m \text { of GuarNoW } \Rightarrow m d s \text { GuarNoW }- \text { varsNoW } k \\
& \text { AsmNoW } \Rightarrow m d s \text { AsmNoW } \cup \text { vars NoW } k \\
& \text { | GuarNoRW } \Rightarrow \text { mds GuarNoRW - varsNoRW } k \\
& \mid \mathbf{A s m N o R W} \Rightarrow m d s \mathbf{A s m N o R W} \cup \operatorname{varsNoRW} k
\end{aligned}
$$

The converse occurs when releasing a lock: the thread drops the assumptions it was making about that region of memory, and once again makes guarantees not to access it.

Definition 3.2 (Impact on mode state $m d s$ of releasing lock $k$ ).

$$
\begin{aligned}
& m d s \ominus k \triangleq \lambda m \text {. case } m \text { of GuarNoW } \Rightarrow m d s \text { GuarNoW } \cup \operatorname{varsNoW} k \\
& \text { | AsmNoW } \Rightarrow \text { mds AsmNoW - varsNoW k } \\
& \text { | GuarNoRW } \Rightarrow m d s \text { GuarNoRW } \cup \operatorname{varsNoRW~} k \\
& \mid \mathbf{A s m N o R W} \Rightarrow m d s \text { AsmNoRW - varsNoRW } k
\end{aligned}
$$

The operational semantics for $\operatorname{lock}(k)$ is then given by two rules: LOCKACQ when lock $k$ is available, and LOCKSPIN when it is already held. For these, we use predicate $\operatorname{ev}_{\text {Lock }}::$ $\mathrm{Val} \Rightarrow$ bool with designated constants $\mathrm{True}_{\text {Lock }}, \mathrm{False}_{\text {Lock }}::$ Val to indicate that the lock is, resp. is not held-i.e. $\mathrm{ev}_{\text {Lock }}\left(\operatorname{True}_{\text {Lock }}\right)=\operatorname{True}_{\text {and }} \mathrm{ev}_{\text {Lock }}\left(\mathrm{False}_{\text {Lock }}\right)=$ False ${ }^{3}$

Apart from impacting the mode state as already specified (by Definition 3.1), attempting to acquire an available lock will succeed in the usual manner, setting the lock-variable to the designated constant (True Lock $_{\text {) }}$ ) to prevent subsequent lock acquisition attempts:

$$
\begin{aligned}
& \neg \operatorname{ev}_{\text {Lock }}(\text { mem }(\text { Lock } k)) \quad m e m^{\prime}=\text { mem }\left[\text { Lock } k \mapsto \text { True }_{\text {Lock }}\right] \\
& \frac{m d s^{\prime}=m d s \oplus k}{\langle\operatorname{lock}(k), m d s, m e m\rangle_{\mathrm{w}}{ }^{{ }_{\mathrm{w}}}\left\langle\mathbf{\text { stop}}, m d s^{\prime}, \mathrm{mem}^{\prime}\right\rangle_{\mathrm{w}}} \text { LOCKACQ }
\end{aligned}
$$

Attempting to acquire an already-held lock results in a stuttering evaluation step:

$$
\frac{\operatorname{ev}_{\text {Lock }}(\text { mem }(\text { Lock } k))}{\langle\operatorname{lock}(k), m d s, \text { mem }\rangle_{\mathrm{w}} \rightsquigarrow_{\mathrm{w}}\langle\operatorname{lock}(k), m d s, \text { mem }\rangle_{\mathrm{w}}} \text { LOCKSPIN }
$$

Then, the operational semantics for $\operatorname{unlock}(k)$ is given by two rules, of which only one, LOCKREL, will ever be used by programs that follow locking discipline. This rule requires that the mode state $m d s$ is consistent with the present thread having previously acquired the lock $k$ : In short, it should have all the assumptions, but none of the guarantees, associated with the variables governed by the lock. To specify this, we define the following helper:

Definition 3.3 (Mode state is consistent with holding a lock $k$ ).

$$
\begin{aligned}
& \text { lock-held-mds-correct } m d s k \triangleq \\
& \forall x .(x \in \operatorname{varsNoW} k \longrightarrow x \notin m d s \text { GuarNoW } \wedge x \in m d s \text { AsmNoW }) \wedge \\
& \quad(x \in \operatorname{varsNoRW} k \longrightarrow x \notin m d s \text { GuarNoRW } \wedge x \in m d s \text { AsmNoRW })
\end{aligned}
$$

\footnotetext{
${ }^{3}$ All three of $\mathrm{ev}_{\text {Lock }}$, True $_{\text {Lock }}$, False $_{\text {Lock }}$ are parameters that are set by the user of the theory, with the proviso that their choice of parameters satisfy that $\operatorname{ev}_{\text {Lock }}\left(\operatorname{True}_{\text {Lock }}\right)$ and $\neg \mathrm{ev}_{\text {Lock }}\left(\mathrm{False}_{\text {Lock }}\right)$ hold as required.
} 
With that condition satisfied, the LOCKREL rule specifies that an unlock $(k)$ will proceed successfully, to enact lock release on the memory and mode state as expected:

$$
\begin{aligned}
& \text { lock-held-mds-correct } m d s k \quad m e m^{\prime}=\operatorname{mem}\left[\text { Lock } k \mapsto \text { False }_{\text {Lock }}\right] \\
& \frac{m d s^{\prime}=m d s \ominus k}{\langle\operatorname{unlock}(k), m d s, m e m\rangle_{\mathrm{w}}{ }{ }_{\mathrm{w}}\left\langle\mathbf{s t o p}, m d s^{\prime}, \text { mem }^{\prime}\right\rangle_{\mathrm{w}}} \text { LOCKREL }
\end{aligned}
$$

To ensure that the While evaluation semantics is defined for all possible configurations, the LOCKINVALID rule defines a stuttering evaluation step for attempts to unlock $(k)$ that violate the locking discipline due to not having previously acquired the lock $k$ :

$$
\frac{\neg \text { lock-held-mds-correct } m d s k}{\langle\operatorname{unlock}(k), m d s, m e m\rangle_{\mathrm{w}} \rightsquigarrow_{\mathrm{w}}\langle\mathbf{u n l o c k}(k), m d s, m e m\rangle_{\mathrm{w}}}
$$

As mode state is nominally a form of ghost state, having the operational semantics appear to depend on it in this manner is rather unusual. To remove the semantics' reliance on ghost state, the program developer must use a check for local-mode-compliance (Definition 2.11) that only ever admits programs that satisfy the lock-held-mds-correct check whenever attempting to unlock $(k)$. For such programs, the operational semantics is equivalent to one that (1) omits the lock-held-mds-correct check from the LOCKREL rule, and (2) omits the LOCKINVALID rule from the While-language semantics entirely. An example of such a check is included in our Isabelle/HOL supplement.

\subsection{Restrictions on locking disciplines}

Here we lay out some cleanliness conditions on locking disciplines, giving particular focus to those relevant to our locking semantics (Section 3.1), and to our verification efforts for While's global compositionality property Section 3.3) and our compiler Section 5.

Of these, only one is a hard consequence of the underlying CVDNI theory we presented in Section 2. The per-thread CVDNI property com-secure (Definition 2.7) effectively compels us to enforce that secrets are never allowed to leak into the locking state. Otherwise, mode state would become tainted upon any attempt to acquire a lock whose status is secret, which would violate com-secure's requirement that modes-equality must be maintained at all times (note the $={ }_{m d s}$ enforced by strong-low-bisim-mm, Definition 2.4). To ensure that com-secure will always treat the locking state as an untrusted sink, we impose the following requirement on the $\mathscr{L}$ parameter supplied by the program developer:

Proposition 3.4 ( $\mathscr{L}$ must permanently assign Low classification to all lock-variables $k$ ).

$$
\forall k \text { mem. } \mathscr{L} \text { mem }(\text { Lock } k)=\text { Low }
$$

The remaining restrictions are consequences of various simplifications of convenience.

First, note that the type signature of the lock-interp parameter (given in Section 3.1) only allows locks to govern program variables, not other locks. We justify this simplification with the fact that if some lock $k$ governed lock $k^{\prime}$, then $k$ would already have to be held whenever acquiring $k^{\prime}$-otherwise, the change to $k^{\prime}$ would violate a no-write assumption implied by the locking discipline. This, however, would make $k^{\prime}$ entirely redundant with $k$. 
Second, the lock acquisition and release semantics we gave in Section 3.1 is rather simplified, in that releasing a lock will drop the assumptions of all its variables from the mode state, even if another lock for that variable is still held! Thus, we signal that it only works for disciplines wherein no more than one lock governs each program variable, by asserting:

Proposition 3.5 (No variable can be managed by more than one lock).

$$
\begin{aligned}
& \forall v k . v \in \text { varsNoW } k \cup \operatorname{varsNoRW} k \longrightarrow \\
& \quad\left(\forall k^{\prime} . v \in \text { varsNoW } k^{\prime} \cup \operatorname{varsNoRW} k^{\prime} \longrightarrow k^{\prime}=k\right)
\end{aligned}
$$

We believe that it would be feasible to relax Proposition 3.5, by generalising While's locking semantics to allow disciplines wherein multiple locks must be held to access a given variable. To satisfy CVDNI-preserving refinement (particularly Definition 2.13), a compiler would need to preserve the lock memory operations that implement the more sophisticated bookkeeping needed, as ours does for the current, much simpler locking semantics.

Next, we assume that the program developer has not specified any "vacuous" locks (i.e. ones that govern no variables), and that all locks grant at most one of AsmNoW or AsmNoRW (i.e. not both) on any given variable. These two assumptions allow us to exclude various pathological cases from our reasoning in Section 3.3 and Section 5, respectively:

Proposition 3.6 (Every lock governs access to some variable).

$$
\forall k \text {. varsNoW } k \cup \operatorname{varsNoRW} k \neq \emptyset
$$

Proposition 3.7 (The lock interpretation sets for any given lock $k$ do not overlap).

$$
\forall k \text { varsNoW } k \cap \operatorname{varsNoRW} k=\emptyset
$$

The final two restrictions simplify the possible interactions between locks and control variables: We disallow locks from being control variables, and require variables to be governed by the same lock as their control variables. In particular, they will help us establish (in Section 5.4) that the compiler produces programs that satisfy local-mode-compliance.

First, recall we mentioned that, as part of local-mode-compliance (Definition 2.11, the doesnt-read-(or-modify) assertions entail that any guarantees not to access some variable $v$ will effectively apply also to all of $v$ 's control variables. Disallowing lock-variables from being control variables thus ensures that $\operatorname{lock}(k)$ and $\mathbf{u n l o c k}(k)$, because they only access lock-variable $k$, cannot violate doesnt-read-(or-modify) for any program-variables:

Proposition 3.8 (Lock-variables $k$ cannot be control variables).

$$
\forall k .(\text { Lock } k) \notin \mathscr{C}
$$

Finally, requiring variables to be governed by the same lock as their control variables effectively ensures they are always locked simultaneously. Apart from making it easier for programs to satisfy local-mode-compliance, this also naturally prevents leaks caused by other threads changing a variable's classification to Low when it still contains High data:

Proposition 3.9 (Variables are always governed by the same lock as their control variables).

$$
\begin{aligned}
\forall c v k . \operatorname{Var} c \in \mathscr{C} \operatorname{vars}(\operatorname{Var} v) \longrightarrow & (c \in \operatorname{varsNoW} k=v \in \operatorname{varsNoW} k) \wedge \\
& (c \in \operatorname{varsNoRW} k=v \in \operatorname{varsNoRW} k)
\end{aligned}
$$




\subsection{Proof of global modes compatibility as an invariant}

This section will present proof that global-modes-compatibility (Definition 2.12) holds as an invariant for concurrent While programs (Section 3.3) when initialised to have no locks held (Section 3.4). Consequently, it is sufficient for a developer to use a local compliance check (Sison, 2020) to obtain the sound-mode-use condition (Definition 2.10) needed for per-thread security proofs to be compositional via Theorem 2.8 .

Recall from Section 2.2 that this compatibility requirement formalises that for all reachable global configurations of a concurrent program, any assumptions made by any of the threads must be met by corresponding guarantees made by all of the other threads:

Definition 2.12 (Global modes compatibility).

global-modes-compatibility $g c \triangleq \forall m d s s \in$ reachable-mds-lists $g c$. compatible-modes $m d s s$ where

$$
\begin{aligned}
& \text { reachable-mds-lists } g c \triangleq \\
& \left\{m d s s \mid \exists c m s^{\prime} m e m^{\prime} \text { sched. } g c-_{\text {sched }}\left(\mathrm{cms}^{\prime}, \text { mem }^{\prime}\right) \wedge \text { map mds } c m s^{\prime}=m d s s\right\} \\
& \text { compatible-modes } m d s s \triangleq \forall i x . i<\text { length } m d s s \longrightarrow \\
& (x \in m d s s[i] \text { AsmNoRW } \longrightarrow \\
& \quad(\forall j<\text { length } m d s s . j \neq i \longrightarrow x \in m d s s[j] \text { GuarNoRW })) \wedge \\
& (x \in m d s s[i] \text { AsmNoW } \longrightarrow \\
& \quad(\forall j<\text { length } m d s s . j \neq i \longrightarrow x \in m d s s[j] \text { GuarNoW }))
\end{aligned}
$$

The approach to establish global-modes-compatibility here will be to define three mode management requirements that taken together imply compatible-modes, and to prove them invariant for concurrent While programs when initialised such that they hold to begin with.

The first of these pertains to variables whose access is governed by some lock, according to the locking discipline. To define it, we need, alongside lock-held-mds-correct (Definition 3.3 from Section 3.1, a predicate that specifies the correct mode state for not holding a lock $k$ : It should make all of the guarantees, and have none of the assumptions associated with the variables governed by $k \bigsqcup^{4}$ Stated formally:

Definition 3.10 (Mode state is consistent with not holding a lock $k$ ).

$$
\begin{aligned}
& \text { lock-not-held-mds-correct } m d s k \triangleq \\
& \forall x .(x \in \text { varsNoW } k \longrightarrow x \in m d s \text { GuarNoW } \wedge x \notin m d s \text { AsmNoW }) \wedge \\
& (x \in \text { varsNoRW } k \longrightarrow x \in m d s \text { GuarNoRW } \wedge x \notin m d s \text { AsmNoRW })
\end{aligned}
$$

Note that our simplifying exclusion of "vacuous" locks (Proposition 3.6) ensures we never have to deal with a case where lock-held-mds-correct $m d s k$ and lock-not-held-mds-correct $m d s k$ hold simultaneously.

The requirement on global configurations regarding these lock-managed variables is then as follows: If and only if a given lock is held by anybody, then exactly one thread has a mode state consistent with holding it; furthermore, all other threads will have a mode state consistent with not holding it. Formally, with mdss $g c \triangleq$ map mds (cms $g c$ ):

\footnotetext{
${ }^{4}$ Note that this not merely the negation of lock-held-mds-correct $m d s k$ Definition 3.3)!
} 
Definition 3.11 (Lock-managed variable modes are compatible with memory).

$$
\begin{aligned}
& \text { lock-managed-modes-mem-compatible } g c \triangleq \\
& \forall k \text {. if }\left(\mathrm{ev}_{\text {Lock }}((\mathrm{mem} g c) k)\right) \text { then } \\
& \exists ! i . i<\text { length }(\mathrm{cms} g c) \wedge \\
& \text { lock-held-mds-correct }(\text { mdss } g c)[i] k \wedge \\
& (\forall j<\text { length }(\mathrm{cms} g c) . i \neq j \longrightarrow \\
& \text { lock-not-held-mds-correct }(\text { mdss } g c)[j] k) \\
& \text { else } \forall i<\text { length }(\mathrm{cms} g c) . \\
& \text { lock-not-held-mds-correct }(\text { mdss } g c)[i] k
\end{aligned}
$$

The second requirement pertains to variables whose access is entirely ungoverned by any locks in the locking discipline. For these we specify a more direct check that if any thread in the global configuration has an assumption about access to any of these variables, then all other threads must be providing the corresponding guarantee to that assumption:

Definition 3.12 (Unmanaged variable modes are compatible).

$$
\begin{aligned}
& \text { unmanaged-var-modes-compatible } g c \triangleq \forall i x . i<\text { length }(\text { mdss } g c) \longrightarrow \\
& \begin{aligned}
\left(x \notin \bigcup_{k:: L o c k} \text { varsNoRW } k \longrightarrow\right. \\
(x \in(\text { mdss } g c)[i] \text { AsmNoRW } \longrightarrow \\
\quad(\forall j<\text { length }(\text { mdss } g c) . j \neq i \longrightarrow x \in(\text { mdss } g c)[j] \text { GuarNoRW }))) \wedge \\
\left(x \notin \bigcup_{k:: \text { Lock }} \text { varsNoW } \longrightarrow\right. \\
\quad(x \in(\text { mdss } g c)[i] \text { AsmNoW } \longrightarrow \\
\quad(\forall j<\text { length }(\text { mdss } g c) . j \neq i \longrightarrow x \in(\text { mdss } g c)[j] \text { GuarNoW })))
\end{aligned}
\end{aligned}
$$

Also proved invariant is a third, minor property that enforces globally that no assumptions or guarantees are ever recorded regarding access to lock-variables:

Definition 3.13 (No assumptions and guarantees on lock variables).

$$
\begin{aligned}
\text { no-lock-mds } m d s & \triangleq \forall l m \text {. Lock } l \notin m d s m \\
\text { no-lock-mds-gc } g c & \triangleq \forall m d s \in \text { set (mdss } g c \text { ). no-lock-mds } m d s
\end{aligned}
$$

This follows trivially from (1) our simplification (discussed in Section 3.2 only to allow locks to protect access to program variables and not other locks, and (2) the resulting fact that no While primitives ever touch any mode state pertaining to lock variables. Thus, further details on this third management requirement will be elided.

We then have straightforwardly from their definitions that together, these three mode management requirements imply compatible modes for a given global configuration:

Lemma 3.14 (Management requirements ensure compatibility).

$$
\begin{gathered}
\text { lock-managed-modes-mem-compatible } g c \quad \text { unmanaged-var-modes-compatible } g c \\
\text { no-lock-mds-gc } g c
\end{gathered}
$$


Proofs of invariance then proceed by induction over the single-step evaluation semantics of an arbitrary thread taking a step to progress the system to a new global configuration.

For the first management requirement (Definition 3.11):

Lemma 3.15 (Single-step preservation of lock-managed-modes-mem-compatible).

\begin{tabular}{c} 
lock-managed-modes-mem-compatible $(\mathrm{cms}$, mem $)$ \\
$\left\langle c_{i}, m d s_{i}, m e m\right\rangle_{\mathrm{w}} \rightsquigarrow_{\mathrm{w}}\left\langle c_{i}^{\prime}, m d s_{i}^{\prime}, \text { mem }^{\prime}\right\rangle_{\mathrm{w}} \quad i<$ length $\mathrm{cms}$ \\
$\mathrm{cms} s^{\prime}=c m s\left[i:=\left(c_{i}^{\prime}, m d s_{i}^{\prime}\right)\right] \quad c m s[i]=\left(c_{i}, m d s_{i}\right)$ \\
\hline lock-managed-modes-mem-compatible $\left(\mathrm{cms}^{\prime}, \mathrm{mem}^{\prime}\right)$
\end{tabular}

Proof. By induction over the single-threaded evaluation semantics of the program at index $i$ that is taking a step.

$\operatorname{lock}(k)$ preserves the property because it only allows a thread to set lock $k$ 's memory if it is not already set - it would then become the single unique thread whose mode state is consistent with holding $k$. Otherwise, the mode states and memory remain unchanged.

Similarly, unlock $(k)$ preserves the property because its only possible change is to unset lock $k$ 's memory, and return the unique thread holding lock $k$ to a mode state consistent with not holding $k$.

The other While commands preserve the property because they do not touch the mode state nor any lock-variables.

For the second management requirement (Definition 3.12):

Lemma 3.16 (Single-step preservation of unmanaged-var-modes-compatible).

$$
\begin{gathered}
\text { unmanaged-var-modes-compatible }(\mathrm{cms}, \text { mem }) \\
\left\langle c_{i}, m d s_{i}, m e m\right\rangle_{\mathrm{w}} \rightsquigarrow_{\mathrm{w}}\left\langle c_{i}^{\prime}, m d s_{i}^{\prime}, \text { mem }^{\prime}\right\rangle_{\mathrm{w}} \quad i<\text { length } \mathrm{cms} \\
\mathrm{cms} s^{\prime}=c m s\left[i:=\left(c_{i}^{\prime}, m d s_{i}^{\prime}\right)\right] \quad c m s[i]=\left(c_{i}, m d s_{i}\right) \\
\hline \text { unmanaged-var-modes-compatible }\left(\mathrm{cms}^{\prime}, \mathrm{mem}^{\prime}\right)
\end{gathered}
$$

Proof. Again, by induction over the single-threaded evaluation semantics of the program at index $i$ that is taking a step.

We prove and use lemmas that $\operatorname{lock}(k)$ and $\operatorname{unlock}(k)$ do not touch any mode state pertaining to variables that are unmanaged by any locks, and that the remaining While commands do not touch the mode state at all. Therefore evaluation steps cannot possibly have any effect on the compatibility of modes on these variables.

These single-step evaluation results lift easily to invariance results over the global multistep evaluation semantics quantified over arbitrary schedules. These invariance results, with the fact that the management requirements ensure compatibility (Lemma 3.14, yield in a straightforward manner the desired global compatibility invariance theorem:

Theorem 3.17 (Mode management requirements ensure global compatibility).

$$
\text { lock-managed-modes-mem-compatible } g c \quad \text { unmanaged-var-modes-compatible } g c
$$
no-lock-mds-gc $g c$

$$
\text { global-modes-compatibility } g c
$$




\subsection{Initial conditions ensuring global modes compatibility}

We now define conditions on memory and mode state consistent with no locks being held, and show that initialising a system under these conditions is enough to satisfy the global compatibility part (Definition 2.12) of the sound-mode-use side condition (Definition 2.10) of the compositionality theorem for our security property (Theorem 2.8 ).

We define the following predicate for initial memory:

Definition 3.18 (A requirement for initial memory that no locks are held).

$$
\text { no-locks-held mem } \triangleq \forall k . \neg \mathrm{ev}_{\text {Lock }}(\text { mem } k)
$$

We then define an initial mode state $\mathrm{mds}_{0}::$ Mode $\Rightarrow$ Var set that provides all guarantees demanded by the lock interpretation parameters varsNoW, varsNoRW (described in Section 3.1) for all lock variables in the system, and makes no assumptions:

Definition 3.19 (Initial mode state $\mathrm{mds}_{0}$ ).

$$
\begin{aligned}
& \text { mds }_{0} \triangleq \lambda m \text {. case } m \text { of GuarNoW } \Rightarrow \bigcup_{k:: \text { Lock }} \operatorname{varsNoWk} \\
& \mid \text { GuarNoRW } \Rightarrow \bigcup_{k:: \text { Lock }} \operatorname{varsNoRWk} \\
& \mid \text { AsmNoW } \Rightarrow \emptyset \\
& \mid \text { AsmNoRW } \Rightarrow \emptyset
\end{aligned}
$$

We are then able to show that these conditions are enough to satisfy the requirements we just showed (in Section 3.3 ensure global modes compatibility for While:

Lemma 3.20 (Initialising with no-locks-held, $\mathrm{mds}_{0}$ ensures global modes compatibility).

$$
\frac{\text { no-locks-held } m e m \quad \forall(c, m d s) \in \text { set } c m s . m d s=\mathrm{mds}_{0}}{\text { global-modes-compatibility }(\mathrm{cms}, \mathrm{mem})}
$$

Proof. Theorem 3.17 obliges us to show that the mode management conditions (Definitions 3.11, 3.12, and 3.13 hold. This follows straightforwardly from all the relevant definitions.

\section{Target language: RISC with mutex locks}

Here we introduce RISC with mutex locks (hereafter RISC), the target of our compiler. This is a generic RISC-style assembly language based on the RISC architecture targeted by the compilation scheme of Tedesco et al. (2016). A RISC program text is a list of RISC instructions $I$, each optionally associated with a label:

$$
\begin{aligned}
I::= & {[l:] } \\
B::= & \text { Load } r v \mid \text { Store } v r|\mathbf{J m p} l| \mathbf{J z} l r \mid \text { Nop } \\
& \text { MoveK } r n \mid \text { MoveR } r r \mid \text { Op } \oplus r r \\
& \text { LockAcq } k \mid \text { LockRel } k
\end{aligned}
$$


Here we fix the types of the constant values $n::$ Val, binary arithmetic operators $\oplus::$ $V a l \Rightarrow V a l \Rightarrow V a l$, shared program variables $v::$ Var, and shared lock variables $k::$ Lock to be the same as those for the source While language being compiled. Thus, the only new types here compared to Section 3 are for the register identifiers $r::$ Reg, and labels $l::$ Lab.

RISC has a small-step operational semantics that is largely unchanged from Tedesco et al. (2016), in that each step updates a distinguished program counter register, which captures the current thread's program location as an index into its RISC program text. The instructions MoveK, MoveR, Load, and Store, for moving values to and between the registers and shared memory, and the "no-op" instruction Nop, all increment the program counter; the "jump if zero" instruction $\mathbf{J z} l r$ updates it to the index of the instruction at $l$ if $r$ contains zero (else increments it); the unconditional $\mathbf{J m p} l$ does so unconditionally.

Modifying this instruction set from Tedesco et al. (2016), we then customise the Op instruction, and add LockAcq $k$ and LockRel $k$ instructions, to have semantics mirroring those of $\oplus, \operatorname{lock}(k)$, and $\mathbf{u n l o c k}(k)$ from While respectively. Whereas the RISC equivalents for the LOCKACQ and LOCKREL evaluation rules (described by Section 3.1 for the While language) increment the program counter, those for LOCKSPIN and LOCKINVALID leave it unchanged. There is no RISC evaluation rule that changes the program text.

Although it has only direct-addressing Load and Store instructions, our RISC target language is adequate for implementing all features of While present in Section 3 , with the bigstep semantics of exp replaced by small-step operations on registers. We relegate RISC's full formal semantics to this paper's supplement Isabelle/HOL material.

Our defining LockAcq $k$ and LockRel $k$ to have the same operational semantics on shared memory and mode state as While's $\operatorname{lock}(k)$ and $\operatorname{unlock}(k)$ has two consequences:

- Our compiler will expect the program developer to supply the details of the locking discipline for the While program being compiled, so as to be able to ensure that the RISC program it produces follows the same discipline.

- We then have that global compatibility is invariant for RISC execution, by a nearidentical argument to the one we presented in Section 3.3. when initialised with the conditions we presented in Section 3.4. This presents one option for obtaining RISClevel composition of per-thread noninterference properties; however, invoking it directly will not be necessary when using the compositional whole-system secure refinement method of Section 2.5. (The alternative options and their application will be demonstrated further, respectively in Section 5.4, Section 6.3,

As for While in Section 3, we instantiate here for RISC the CVDNI theory of Murray et al. (2016b) as recalled in Section 2.2, assuming that the underlying concurrency model (e.g. OS, scheduler etc.) prevents one thread from reading the program text of another. For RISC, we furthermore assume that the context switching mechanism ensures effectively that no thread can read or interfere with the contents of the registers (including the program counter) when active for another thread. Based on these assumptions, we model all three of the program counter register's value $p c::$ nat, RISC program text $P::$ I list, and register bank regs $:: \operatorname{Reg} \Rightarrow V a l$, as thread-private state in the local configuration triple: $\langle((p c, P), r e g s), m d s, m e m\rangle_{\mathrm{r}}$. (We use the subscript ${ }_{\mathrm{r}}$ to distinguish RISC triples.) 


\section{Verified secure compiler for mixed-sensitivity concurrent While programs}

This section presents the COVERN wr-compiler: the first compiler proved to preserve proofs of noninterference for mixed-sensitivity concurrent programs. By using assume-guarantee modes (Mantel et al. 2011) and the decomposition principle of Section 2.4 to prove it introduces (resp.) no race conditions or timing leaks, we demonstrate the applicability to compiler verification of the CVDNI-preserving refinement notion of Section 2.3 originally posed by Murray et al. (2016b). Here the decomposition principle (Figure 4) is crucial because, in separating the concern of preventing new timing leaks, it avoids directly having to prove the cube-shaped refinement diagram (Figure 3) arising from its need to preserve a 2-safety hyperproperty (Terauchi \& Aiken, 2005; Clarkson \& Schneider, 2010).

To preserve security for mixed-sensitivity concurrent programs, CVDNI-preserving refinement demands small-step preservation of the contents of all shared memory locations including those that control value-dependent classifications and implement locks. As it is unusual for verified compilers to make such promises, we show that a valid approach is to take advantage of CVDNI's assume-guarantee framework to:

1. test and preserve any absence of race conditions implied (via the framework) by mutex lock-based synchronisation of access to such locations, and then

2. use this absence of race conditions to establish the small-step preservation of their contents demanded for security-preserving refinement.

In doing so, we prove that some optimisations the wr-compiler performs with its knowledge of the locking discipline-it avoids unnecessary Loads and recalculation of common subexpressions over shared memory when locked - are safe to allow without violating CVDNI.

In preserving CVDNI, the wr-compiler preserves security proofs that are produced by the program verification techniques of Sison (2020) for While with mutex locks, which in turn were adapted from Murray et al. $(2016 \mathrm{~b}$ c). We will present such an application of our compiler, to a case study program verified using these techniques, in Section 6 .

Section 5.1 will focus on the wr-compiler's particular adaptations to CVDNI (beyond the fault-resilient noninterference targeted by the original compilation scheme of Tedesco et al. (2016)), in the form of static checks and invariants that (resp.) test for and maintain the absence of race conditions on lock-protected shared variables. Section 5.2 formalises a ban, preserved by the wr-compiler, on secret-dependent control flow. Section 5.3 then presents formal proof (structured by our decomposition principle of Section 2.4) that the wrcompiler implements CVDNI-preserving refinement. Section 5.4 ultimately presents proofs of overall security preservation results useful to users of the wr-compiler: Namely, it can be used either to preserve security down to RISC for an entire concurrent While-language program, or to preserve the per-thread security for threads that will be run alongside others written directly in the RISC-language.

\subsection{Preserving race-free expression evaluation}

Recall from Section 2.3 that CVDNI-preserving refinement (Murray et al. 2016b) demands that all shared memory contents be preserved, between each target- and source-language 
configuration that it relates. This is security critical for mixed-sensitivity concurrent programs, as it ensures that any future influence of those contents on value-dependent classifications (via control variables) or readability by other threads (in the case of the While and RISC languages, via lock variables) is preserved.

The wr-compiler's approach to preserving the contents of shared memory is to ensure:

1. That values calculated by expressions are preserved by compilation-that is, they have the same value when written back to shared memory (or conditionally branched on) by the RISC program, as they did in the original While program; and

2. That expression evaluation is race-free-that is, free of any race conditions with other threads that would render the calculated expression inaccurate.

To this end, the wr-compiler requires of the original While program that whenever each thread attempts to evaluate an expression, it must hold locks ensuring the stability of all variables referenced by the expression.

Thus, its knowledge and enforcement of the locking discipline is crucial, not only to show that its optimisations preserve CVDNI, but that any meaningful operation over shared memory preserves it. It therefore tests for and rejects programs that exhibit potential race conditions due to their failure to follow locking discipline- these result in a failed compilation.

The wr-compiler tracks two kinds of information to achieve these outcomes: the contents of registers as expressions over shared variables, and assumptions on access to variables by other threads. The structures the wr-compiler uses to do this are, respectively:

- A register record $\Phi:: \operatorname{Reg} R e c \triangleq R e g \rightarrow \exp$. This draws inspiration from that used by the compilation scheme of Tedesco et al. (2016) (originally of type Reg $\rightarrow$ Var) to avoid generating unnecessary Load instructions to registers that already contain a variable; in addition, here we extend it to track entire expressions on shared variables.

- An assumption record $\mathscr{S}::$ AsmRec $\triangleq($ Var set $\times$ Var set $)$ that tracks which variables at a given point in the source While program are "stable" due to having, respectively, an AsmNoW or AsmNoRW assumption.

The wr-compiler's main function compile-cmd then outputs every register-assumption record pair (or compilation record) $C=(\Phi, \mathscr{S})::$ CompRec $\triangleq \operatorname{RegRec} \times A s m R e c$ associated with the program state before execution of each instruction in the output RISC program 5 A typical invocation to compile some $c:: \mathrm{cmd}$ takes an initial compilation record $C$, and returns the CompRec-annotated RISC program PCs :: $(I \times C o m p R e c)$ list (i.e. map fst PCs recovers an unannotated RISC text), and a final compilation record $C^{\prime}$ :

Example 5.1 (Example invocation of the COVERN wr-compiler).

$$
\left(P C s, l^{\prime}, n l^{\prime}, C^{\prime}, \text { failed }\right)=\text { compile-cmd } C l n l c
$$

The remainder of this section will focus on formal properties of the compilation records output alongside each RISC text: Section 5.1.1 will elaborate on checks enforced on input

\footnotetext{
${ }^{5}$ For readability, we will use regrec, asmrec to denote a CompRec's (resp.) fst, snd projections.
} 
programs with the help of AsmRecs, and Section 5.1.2 will present a resulting property that $R e g R e c s$ track stable expressions, needed to prove security preservation (in Section 5.3).

Remaining details (e.g. $l, l^{\prime}, n l, n l^{\prime}$ for label allocation) will be relegated to appendices. We note here only that (1) compile-cmd may return True for failed to reject the input program, such as when it detects a race condition (described further in Section 5.1.1), or if expression depth exceeds the limit assumed by the register allocation scheme model (elided to Appendix B; ; also, (2) relative to the label allocation scheme (elided to Appendix A) we proved that the control flow of each program fragment compiled by the wr-compiler remains self-contained even when composed sequentially with other such fragments.

\subsubsection{Requirements on inputs to the wr-compiler}

We define a shared variable $v$ to be recorded as assumed stable if it and all its control variables (i.e. $\mathscr{C}$ vars $v$ ) cannot presently be written to by another thread-that is, if they are recorded as having either of AsmNoW or AsmNoRW active on them. Formally:

Definition 5.1 (Stability of variable $v$ according to assumption record $\mathscr{S}$ ).

$$
\text { var-stable } \mathscr{S} v \triangleq v \in(\text { fst } \mathscr{S} \cup \text { snd } \mathscr{S}) \wedge\left(\forall v^{\prime} \in \mathscr{C} \text { vars } v \cdot v^{\prime} \in(\text { fst } \mathscr{S} \cup \text { snd } \mathscr{S})\right)
$$

For register record entries to be of any help in ensuring consistency of While and RISC expression evaluation, we exclude expression evaluation on race-prone variables by lifting the concept of stability to register records. The following predicate asserts internal consistency of the compilation record $C$ created by compile-cmd, in the sense that the register record may only map to expressions that mention variables that are recorded as stable by the assumption record accompanying it. (Here, ran denotes the range of a map.)

Definition 5.2 (Stability of the register record in compilation record $C$ ).

$$
\text { regrec-stable } C \triangleq \forall e \in \operatorname{ran}(\operatorname{regrec} C) .(\forall v \in \exp \text {-vars } e \text {. var-stable }(\operatorname{asmrec} C) v)
$$

We then implement a collection of stability-checks :: cmd $\times$ CompRec $\Rightarrow$ bool (called no-unstable-exprs in Sison \& Murray (2019)) as a recursive function on the structure of While programs, that compile-cmd will use to ensure the following requirements of the given $\mathrm{cmd}$ if started with a configuration consistent with the given CompRec:

- The first two requirements ensure that programs comply with the locking discipline:

- The requirement regarding reading from shared variables establishes the main outcome of freedom from race conditions we described at the beginning of Section 5.1: The program must not refer to expressions on any unstable variables.

As a matter of convenience, instead of introducing a dedicated primitive to the While language for reading atomically from a single (otherwise-unstable) device memory location, in our case study model of Section 6 we model such interactions using a simple assignment $x:=y$ protected by a "read-atomicity" lock on a shared memory location $y$ that models the hardware interface $f^{6}$

\footnotetext{
${ }^{6}$ When such atomic hardware primitives exist on a given architecture, we expect it would be straightforward for source languages to expose them and oblige their architecture-specific compilers to compile them to that single atomic instruction in the target language's semantics, which would eliminate the need for such locks.
} 
- If the program assigns to an unstable shared variable, then it must not be a lockgoverned one according to the locking discipline. This prevents the violation of any guarantees not to write to the variable (due to not holding its lock).

- The remaining two requirements follow some simplifying assertions, originally made by the security type system of Murray et al. (2016b), that ensure mode state remains consistent after conditional branching and looping:

- The two sides of any if-conditional branches in the program must both end with, effectively, the same set of locks held - to be precise, judging by their effect on the mode state, as captured by the assumption record.

- For similar reasons, we require any while-loops in the program to restore the original set of locks held on loop entry (again, as captured by the assumption record) on loop termination.

We believe these to be reasonable simplifications given that, in our setting, the set of variables governed by each lock does not change at runtime in such a way that would require access to them to be lock protected (or not) in a conditional manner.

Together, regrec-stable $C$ and stability-checks $c C$ make up the main two requirements of a predicate compile-cmd-input-reqs $C l n l c$ imposed on the input arguments to compile-cmd. (Its other two requirements reflect that the terminated While program stop has no valid compilation, and that the initial label, if provided, must be valid-see Appendix A) If any of these requirements are violated, compile-cmd rejects the program with failed $=$ True:

Definition 5.3 (Requirements on inputs to compile-cmd).

$$
\begin{array}{r}
\text { compile-cmd-input-reqs } C l n l c \triangleq \text { stability-checks } c C \wedge \text { regrec-stable } C \wedge \\
c \neq \text { stop } \wedge(\forall x . l=\text { Some } x \longrightarrow x<n l)
\end{array}
$$

\subsubsection{Proof that all tracked register contents are stable}

Imposing the predicate compile-cmd-input-reqs Definition 5.3 gives us enough information to prove a lemma that compile-cmd only ever outputs stable register records, that attest to the fact that registers contain the results of evaluating expressions on stable variables.

Stated more precisely, every RISC program returned by a successful invocation of compile-cmd is annotated by CompRecs all with stable register records, and furthermore that the final CompRec's register record is also stable:

Lemma 5.4 (Successful compilations output only stable register records).

$$
\left(P C s, l^{\prime}, n l^{\prime}, C^{\prime}, \text { False }\right)=\text { compile-cmd } C l n l c \quad \text { compile-cmd-input-reqs } C l n l c
$$

$(\forall p c<$ length $P C s$. regrec-stable (map snd $P C s)[p c]) \wedge$ regrec-stable $C^{\prime}$

Proof. By induction on the structure of the While language program $c$, making reference to the implementation of compile-cmd.

For cases that must compile expressions, we furthermore prove and make use of a lemma by induction on the structure of expressions, making reference to the implementation of the 
expression compiler function compile-expr called by compile-cmd. In essence, we prove that (sub)expressions appearing in register records must be stable, for two reasons:

First, they are always only ever subexpressions over variables that must have been stable in the input program when their contents were first loaded into registers.

Second, when compiling an $\operatorname{unlock}(k)$, the wr-compiler will always flush all register records that make reference to any variables that the unlock $(k)$ makes unstable.

\subsection{Preserving a ban on secret-dependent control flow}

The wr-compiler assumes that input While programs have no conditional branches on High-sensitivity values (High-branching), and therefore no secret-dependent control flow. This is a restriction commonly applied as a means to prevent all implicit flows, including timing leaks. This restriction will then be preserved by the wr-compiler for its output RISC programs, reflected primarily in the design of the concrete coupling invariant $\mathscr{I}_{\mathrm{wr}}$ (see Section 5.3.3.

Specifically, the wr-compiler assumes that the confidentiality of input While programs is witnessed by a strong low-bisimulation modulo modes with an extra requirement (supplied as a parameter, as in Section 2.2 that effectively disallows any present or past Highbranching. Relying on the fact that a low-bisimulation already asserts Low-equivalence of memories, the extra requirement asserts that it furthermore pairs only configurations at the same program location, and that any if-conditional expressions must evaluate to the same value in both configurations' memories. Here, the helper function leftmost-cmd gives the leftmost in a sequence of ;-separated While-language commands:

Definition 5.5 (An extra requirement for low-bisimulations $\mathscr{B}$ to ban High-branching).

$$
\begin{aligned}
& \text { no-high-branching } \mathscr{B} \triangleq \\
& \forall c c^{\prime} m d s \text { mem mem }{ }^{\prime} .\left(\langle c, m d s, m e m\rangle_{\mathrm{w}},\left\langle c^{\prime}, \text { mds, } \text { mem }^{\prime}\right\rangle_{\mathrm{w}}\right) \in \mathscr{B} \longrightarrow c=c^{\prime} \wedge \\
& \quad\left(\forall e c_{1} c_{2} \text {. leftmost-cmd } c=\text { if e then } c_{1} \text { else } c_{2} f \longrightarrow \operatorname{ev}_{\exp } m e m e=\operatorname{ev}_{\exp } m e m^{\prime} e\right)
\end{aligned}
$$

Then, in Section 5.4, we will prove that the wr-compiler produces confidential RISC programs with no secret-dependent control flow, as witnessed by a low-bisimulation that asserts a similar extra requirement for RISC programs. In effect, this is the pc-security notion of Molnar et al. (2006), but also explicitly equating the program text:

Definition 5.6 (A pc-security-like requirement for RISC bisimulations $\mathscr{B}$ ).

$$
\begin{aligned}
& \text { pc-security } \mathscr{B} \triangleq \forall p c p c^{\prime} P P^{\prime} \text { regs regs' mds mem mem }{ }^{\prime} . \\
& \left(\langle((p c, P), \text { regs }), m d s, \text { mem }\rangle_{\mathrm{r}},\left\langle\left(\left(p c^{\prime}, P^{\prime}\right), \text { regs }^{\prime}\right), \text { mds }, \text { mem }^{\prime}\right\rangle_{\mathrm{r}}\right) \in \mathscr{B} \longrightarrow p c=p c^{\prime} \wedge P=P^{\prime}
\end{aligned}
$$

\subsection{Use of the decomposition principle}

Having covered the most relevant aspects of the wr-compiler's implementation, we now present the refinement relation $\mathscr{R}_{\mathrm{wr}}$ (in Section 5.3.1), pacing function abs-steps ${ }_{\mathrm{wr}}$ (in Section 5.3.2, and concrete coupling invariant $\mathscr{I}_{\text {wr }}$ (in Section 5.3.3, parameters we use to apply the decomposition principle we presented in Section 2.4 to prove (in Section 5.3.4) that 
successful compilations are legitimised by secure-refinement (Definition 2.15) - the desired confidentiality-preserving notion of refinement for mixed-sensitivity concurrent programs.

The strategy laid out by the decomposition principle will be to prove that these parameters satisfy decomp-refinement-safe (Definition 2.19) for a targeted class of input Whilelanguage programs - ones with no secret-dependent control flow, as we specified in Section 5.2-meaning (for such programs) we can use the parameters to enforce that wrcompiler introduces no secret-dependent inconsistencies in termination, timing behaviour, or assume-guarantee modes.

In doing so we avoid a direct proof of the cube-shaped refinement diagram (Figure 3 ) of Murray et al. (2016b) — which would have involved reasoning about both $\mathscr{R}_{\mathrm{wr}}$ and $\mathscr{I}_{\mathrm{wr}}$ at once-and instead prove (with the assistance of abs-steps ${ }_{w r}$ ) a square-shaped refinement diagram for $\mathscr{R}_{\mathrm{wr}}$ (Figure 4a) more typically found in compiler verification.

\subsubsection{Refinement relation $\mathscr{R}_{\mathrm{wr}}$ and its invariants}

In this section we introduce the refinement relation $\mathscr{R}_{\mathrm{wr}}$ that characterises compilation of programs from While to RISC using the wr-compiler, and prove it satisfies the two properties demanded of $\mathscr{R}_{\mathrm{wr}}$ (alone) by formal secure-refinement (Definition 2.15):

1. Preservation of modes and all contents of shared memory (preserves-modes-mem, Definition 2.13, and

2. Closedness under changes by other threads (closed-others, Definition 2.14).

An actual proof of refinement (using the square-shaped diagram of Figure 4a) for $\mathscr{R}_{\text {wr }}$ will be deferred to Section 5.3.2, which introduces the abs-steps ${ }_{w r}$ function pacing it.

Just like the earlier example of a secure refinement relation (in Figure 2), the refinement relation $\mathscr{R}_{\text {wr }}$ pairs abstract (here, While-language) with concrete (here, RISC-language) program configurations. For example, the if_expr case of $\mathscr{R}_{\mathrm{wr}}$ relates the expressionevaluation part of the While command if $e$ then $c_{1}$ else $c_{2} \mathbf{f i}$, with the corresponding part of the RISC program obtained by running compile-cmd on it, including the conditional jump $\mathbf{J z}$ after expression evaluation. (This case is depicted in Figure 10, and a relevant excerpt of the compile-cmd implementation provided in Figure 11 for comparison, both on page 63 of Appendix C. An informal description of all the cases of $\mathscr{R}_{\mathrm{wr}}$, their purpose, and the invariants they maintain, can also be found in Appendix C.)

We define almost all the cases of $\mathscr{R}_{\mathrm{wr}}$ to assert at least one successful run of compile-cmd (where failed $=$ False). We then define a guard that we impose to restrict the scope of $\mathscr{R}_{\text {wr }}$ only to consider local program configurations consistent with the relevant compilation record produced by compile-cmd. In short, this ensures the actual values in the register bank regs equal any expression the register record says they should have, as evaluated under the current mem; and furthermore, that the assumption record is consistent with the AsmNoW and AsmNoRW modes in the actual mds. Formally: 
Definition 5.7 (Configuration consistency requirements for compiled commands).

compiled-cmd-config-consistent $C$ regs $m d s$ mem $\triangleq$

regrec-mem-consistent $($ regrec $C$ ) regs mem $\wedge$ asmrec-mds-consistent $($ asmrec $C) m d s$ where

regrec-mem-consistent $\Phi$ regs mem $\triangleq \forall r e . \Phi r=$ Some $e \longrightarrow$ regs $r=\mathrm{ev}_{\exp }$ mem $e$

(Consistency between register record, register bank, and shared memory)

asmrec-mds-consistent $\mathscr{S} m d s \triangleq \mathscr{S}=(m d s$ AsmNoW, $m d s$ AsmNoRW $)$

(Consistency between an assumption record and a mode state)

Apart from using compiled-cmd-config-consistent to restrict the scope of $\mathscr{R}_{\mathrm{wr}}$ in this manner, we will also impose it in Section 5.3.4 as initial configuration requirements for compiled programs: Only configurations obeying them may be used to initialise a RISC program compiled by the wr-compiler with initial CompRec C.

The cases of $\mathscr{R}_{\text {wr }}$ also tend to assert regrec-stable (Definition 5.2, which we already proved holds for all compilation records produced by the wr-compiler (Lemma 5.4.

Finally, whenever a case of $\mathscr{R}_{\mathrm{wr}}$ is inductive (e.g. the if_expr case, for its nested calls to compile-cmd for each of its "then" and "else" branches) it quantifies over all configurations that obey compiled-cmd-config-consistent (Definition 5.7) and regrec-stable (Definition 5.2) relative to the initial compilation record given to each nested call to compile-cmd.

With $\mathscr{R}_{\text {wr }}$ thus specified, we can now prove the two requirements for secure-refinement that pertain to $\mathscr{R}_{\mathrm{wr}}$ alone: preserves-modes-mem (Definition 2.13), and closed-others (Definition 2.14). In short, preserves-modes-mem is largely enforced by the definition of $\mathscr{R}_{\mathrm{wr}}$, but closed-others relies in part on $\mathscr{R}_{\mathrm{wr}}$ only ever talking about stable register records:

Lemma 5.8 ( $\mathscr{R}_{\mathrm{wr}}$ preserves modes and memory).

$$
\text { preserves-modes-mem } \mathscr{R}_{\mathrm{wr}}
$$

Proof. By induction on the structure of $\mathscr{R}_{\mathrm{wr}}$.

For all cases of $\left(l c_{w}, l c_{r}\right) \in \mathscr{R}_{\mathrm{wr}}, l c_{w}={ }_{\text {mds }}^{\text {mem }} l c_{r}$ is either asserted directly by the guards or obtainable from the inductive hypothesis.

Lemma 5.9 ( $\mathscr{R}_{\mathrm{wr}}$ is closed under changes by others).

$$
\text { closed-others } \mathscr{R}_{\mathrm{wr}}
$$

Proof. By induction on the structure of $\mathscr{R}_{\mathrm{wr}}$.

Changes by others Definition 2.14 only modify writable variables the same way for both configurations, so preservation of $=\underset{\mathrm{mds}}{\mathrm{mem}}$ is immediate. Also, regrec-mem-consistent is unaffected because by Lemma 5.4, compile-cmd only creates regrec-stable recordsi.e. referring to no writable variables. No other $\mathscr{R}_{\mathrm{wr}}$ guards mention shared memory.

\subsubsection{Refinement pacing function abs-steps $\mathrm{w}_{\mathrm{wr}}$}

In this section we nominate a pacing function, abs-steps ${ }_{w r}$, specifying the number of evaluation steps with which a While program should simulate each step of the RISC program 
to which the wr-compiler compiled it. Using the square-shaped "refinement preservation" diagram of Figure 4a (part of Definition 2.18, we then prove that the $\mathscr{R}_{\mathrm{wr}}$ relation we introduced in Section 5.3.1 is a refinement when "paced" by abs-steps ${ }_{w r}$ in this manner.

Here we define abs-steps ${ }_{w r}$ to depend only on the current program location; consequently, as long as the wr-compiler introduces no secret-dependent control flow, it will also introduce no timing leaks - that is, no secret-dependent variations to the pacing of the program, as disallowed by Figure 4b (part of Definition 2.19) — which we will be obliged to prove in Section 5.3.4 To this end, abs-steps ${ }_{\mathrm{wr}}$ primarily looks at the form of the RISC instruction (sometimes While command) about to be executed, dividing them into three categories:

- Instructions output by compile-expr: Load, Op, and MoveK. For these, abs-steps ${ }_{w r}$ returns 1 if the leftmost-cmd (the leftmost in a sequence of ;-separated commands) of the While program is "while $e$ do $c$ od", to allow it to step to "if $e$ then $(c$; while $e$ do $c$ od) else stop fi" concurrently with the first RISC step of the compiled expression itself. Otherwise, abs-steps ${ }_{w r}$ returns 0 , to indicate the While program standing still while the RISC program takes new steps to evaluate the expression.

- "Epilogue" steps: Jmp and Nop when used for control flow at the end of a smaller compiled program in the context of a larger one. For these, abs-steps $\mathrm{wr}_{\mathrm{r}}$ returns 0.

- All other RISC instructions are assumed to proceed at a lockstep pace with the While command they were compiled from, and for these abs-steps ${ }_{w r}$ returns 1.

Having nominated abs-steps ${ }_{\mathrm{wr}}$ and $\mathscr{R}_{\mathrm{wr}}$, we now have the parameters over which we are obliged, by secure-refinement-decomp (Definition 2.18), to prove refinement preservation (Figure 4a). To this end, we prove firstly that every step of execution of a RISC program, produced by the wr-compiler from a While program, maintains the consistency demanded by compiled-cmd-config-consistent between configurations and compilation records:

Lemma 5.10 (Successfully compiled programs maintain config consistency requirements).

$$
\begin{gathered}
\left(P C s, l^{\prime}, n l^{\prime}, C^{\prime}, \text { failed }\right)=\text { compile-cmd } C l n l c \quad \text { compile-cmd-input-reqs } C l n l c \\
\text { failed }=\text { False } \quad p c<\text { length } P C s \quad P=\text { map fst } P C s \quad C s=\text { map snd } P C s \\
\text { compiled-cmd-config-consistent } C s[p c] \text { regs } m d s \text { mem } \\
\left.\langle((p c, P), \text { regs }), m d s, \text { mem }\rangle_{\mathrm{r}} \rightsquigarrow_{\mathrm{r}}\left\langle\left(\left(p c^{\prime}, P\right), \text { regs }^{\prime}\right), \text { mds }^{\prime}, \text { mem }^{\prime}\right\rangle_{\mathrm{r}}\right)
\end{gathered}
$$

compiled-cmd-config-consistent (if $p c^{\prime}<$ length $P$ then $C s\left[p c^{\prime}\right]$ else $C^{\prime}$ ) regs ${ }^{\prime}$ mds $^{\prime}$ mem $^{\prime}$

Proof. Unfolding Definition 5.7, we in fact prove it separately for regrec-mem-consistent and asmrec-mds-consistent, both times by induction on the structure of While program $c$.

In each case, we use the simplifiers for the compile-cmd implementation to yield the corresponding RISC program fragment in question, and then prove the lemma for each of the possible locations of $p c$ in the compiled program. For both proofs, there is some trickiness in accounting for (and ruling out) which destination $p c^{\prime}$ must be considered for each of these cases of $p c$, particularly for those While programs that compile to RISC programs that may have jumps in them.

Control flow trickiness aside, the intuition for regrec-mem-consistent is that it tests the correctness of the compilation of expressions. For this we prove a sublemma for maintenance of compiled-cmd-config-consistent, by induction on the structure of expressions $e$ 
that are encountered in the While programs if $e$ then $c_{1}$ else $c_{2}$ fi, while $e$ do $c^{\prime}$ od, and $v:=e$. Additionally, $\mathbf{u n l o c k}(k)$ flushes register record entries mentioning variables that are to become unstable, and while $e$ do $c^{\prime}$ od conservatively flushes entries to force evaluation of the loop condition expression. This is safe trivially because flushing entries can never make a consistent register record inconsistent. The rest of the cases for $c$ are straightforward because they do not touch the register record.

Then for asmrec-mds-consistent, the substantial part of the proof is as a test of the correctness of the compiler's bookkeeping of assumptions being consistent with the semantics of $\operatorname{lock}(k)$ and $\operatorname{unlock}(k)$. The other cases for $c$ do not touch the mode state.

Also, we must prove a correctness lemma for the expression compiler:

Lemma 5.11 (Correctness of the expression compiler).

$$
\left(P C s, r, C^{\prime}, \text { False }\right)=\text { compile-expr } C A l e \Longrightarrow\left(\text { regrec } C^{\prime}\right) r=\text { Some } e
$$

Proof. By induction on the structure of expressions $e$, using the simplification rules for the implementation of compile-expr, and also relying on assumptions of correctness of the register allocation scheme supplied by the instantiator of the theory.

Armed with these facts, we can now prove the main refinement preservation result:

Lemma $5.12\left(\mathscr{R}_{\mathrm{wr}}\right.$ is a refinement paced by abs-steps $\left.{ }_{\mathrm{wr}}\right)$.

$$
\begin{aligned}
& \forall l c_{w} l c_{r} \cdot\left(l c_{w}, l c_{r}\right) \in \mathscr{R}_{\mathrm{wr}_{\mathrm{r}} \longrightarrow(} \longrightarrow\left(c_{r}^{\prime} . l c_{r} \rightsquigarrow_{\mathrm{r}} l c_{r}^{\prime} \longrightarrow\right. \\
& \left.\quad\left(\exists l c_{w}^{\prime} . l c_{w} \rightsquigarrow_{\mathrm{w}}^{\left(\mathrm{abs}^{-s t e p s_{\mathrm{wr}}} l c_{w} l c_{r}\right)} l c_{w}^{\prime} \wedge\left(l c_{w}^{\prime}, l c_{r}^{\prime}\right) \in \mathscr{R}_{\mathrm{wr}}\right)\right)
\end{aligned}
$$

Proof. By induction on the structure of $\mathscr{R}_{\mathrm{wr}}$. (Refer to Appendix C for an informal description of all cases of $\mathscr{R}_{\mathrm{wr}}$.)

The base case stop is immediate, as it pertains to a terminated While and RISC program. The base cases that proceed in one step to a terminating program configuration (skip_nop, assign_store, lock_acq, lock_rel) are fairly straightforward because after dealing with the single step, the resulting obligation can then be handled by the stop case. This leaves the last remaining base case assign_expr, which proceeds in one step either to itself, or to assign_store. In all these cases, we use Lemma 5.10 to obtain the preservation of the guards demanded by the $\mathscr{R}_{\mathrm{wr}}$ introduction rule for the destination configuration of the step. Particularly, the assign_store case must make use of regrec-mem-consistent and the correctness of compile-expr (Lemma 5.11) to ensure that once the evaluated expression is written back to shared memory, $l c_{w}^{\prime}={ }_{\text {mds }}^{\text {mem }} l c_{r}^{\prime}$ holds as demanded by the stop case.

The inductive cases that concern expression evaluation (if_expr, while_expr) are much like assign_expr in that they have the possibility of progressing in one step to themselves. Unlike assign_expr however, their other possibility is a conditional jump based on the result of that expression. Again we use Lemma 5.11 to obtain that the result is an accurate calculation of the expression, and this time we prove by the two different cases whether if_expr ends up in if_c1 or if_c2, or if while_expr ends up in while_inner or at stop (having jumped to the exit label). In these cases, the guards over which the inductive references to $\mathscr{R}_{\mathrm{wr}}$ have been quantified are versatile enough to discharge themselves (when 
*_expr steps to itself), or to discharge any reachable initial starting state for the nested compiled RISC program, given that Lemma 5.10 ensures the invariance of these guards.

This just leaves the inductive cases that pertain to configurations inside a nested compiled RISC program (if_c1, if_c2, while_inner), or at the end of one (epilogue_step, while_loop). In these cases, the inductive hypotheses obtained from the inductive reference to $\mathscr{R}_{\mathrm{wr}}$ are always enough to satisfy the guards demanded by the possible destination cases. Like in the proof of Lemma 5.10, the trickiness mostly comes from accounting for all the possible cases of control flow (ruling out spurious destinations) that need to be considered.

\subsubsection{Concrete coupling invariant $\mathscr{I}_{\text {wr }}$}

The next element needed is the concrete coupling invariant $\mathscr{I}_{\mathrm{wr}}$. Recall from Section 5.2 that the no-high-branching requirement (Definition 5.5) ensures that input While programs have no secret-dependent control flow; here we choose $\mathscr{I}_{\text {wr }}$ to ensure that the wr-compiler has not introduced any new secret-dependent control flow in the output RISC program.

We define $\mathscr{I}_{\mathrm{wr}}$ formally to assert that the witness strong low-bisimulation (modulo modes) to be derived for the output program only pairs local configurations that are at the same location $p c=p c^{\prime}$ of the same RISC program $P=P^{\prime}$ :

Definition 5.13 (Concrete coupling invariant $\mathscr{I}_{\mathrm{wr}}$ for compiled programs).

$$
\mathscr{I}_{\mathrm{wr}} \triangleq\left\{\left(\langle((p c, P), r e g s), m d s, m e m\rangle_{\mathrm{r}},\left\langle\left(\left(p c^{\prime}, P^{\prime}\right), r e g s^{\prime}\right), m d s^{\prime}, \mathrm{mem}^{\prime}\right\rangle_{\mathrm{r}}\right) \mid(p c, P)=\left(p c^{\prime}, P^{\prime}\right)\right\}
$$

From this definition, pc-security (Definition 5.6) is clearly immediate for any concrete bisimulation $\mathscr{B}_{\text {C }}$ of $\mathscr{B} \mathscr{R} \mathscr{I}_{\text {wr }}$ Definition 2.16) derived using $\mathscr{I}_{\text {wr }}$.

\subsubsection{Proof of CVDNI-preserving refinement}

With $\mathscr{R}_{\mathrm{wr}}$, abs-steps $\mathrm{wr}_{\mathrm{w}}$, and $\mathscr{I}_{\mathrm{wr}}$ nominated, we are ready to prove confidentialitypreserving refinement using the decomposition principle secure-refinement-decomp (Definition 2.18.

To this end, we now prove the suitability of these three parameters, for While programs that do not branch on High-sensitivity values (as we specified earlier, in Section 5.2):

Lemma 5.14 ( $\mathscr{R}_{\mathrm{wr}}$, abs-steps $\mathrm{wr}_{\mathrm{w}}, \mathscr{I}_{\mathrm{wr}}$ are safe for secure-refinement decomposition).

$$
\frac{\text { strong-low-bisim-mm } \mathscr{B} \quad \text { no-high-branching } \mathscr{B}}{\text { decomp-refinement-safe } \mathscr{B} \mathscr{R}_{\text {wr }} \mathscr{I}_{\text {wr }} \text { abs-steps }{ }_{\text {wr }}}
$$

Proof. Unfolding Definition 2.19 gives us the following obligations. (See also Figure 4,)

For consistent stopping behaviour, we prove a lemma that RISC programs stop if and only if their $p c$ is outside the program text $P$, i.e. $p c>$ length $P$. Because $\mathscr{I}_{\text {wr }}$ equates $p c$ and $P$ for the two configurations, then clearly both have identical stopping behaviour.

For consistency of change in timing behaviour, abs-steps ${ }_{w r}$ depends only on while and RISC program locations, and no-high-branching and $\mathscr{I}_{\text {wr }}$ forces them (respectively) to be equal for the local configurations under consideration. 
For closedness of $\mathscr{I}_{\text {wr }}$ under lockstep execution, the only non-straightforward cases to consider are conditional branching, and the locking primitives. For conditional branching, we use no-high-branching for $\mathscr{B}$ with memory preservation via $\mathscr{R}_{\mathrm{wr}}$ (Lemma 5.8) to ensure that the conditional branching outcome is the same on both sides.

Finally, as the only operations that touch mode state, the locking primitives are the only non-straightforward cases for modes-equality maintenance under lockstep execution. As all lock memory is classified Low (Proposition 3.4), we use strong-low-bisim-mm for $\mathscr{B}$ with memory preservation via $\mathscr{R}_{\mathrm{wr}}$ to ensure the RISC configurations behave consistently.

Lemma 5.15 ( $\mathscr{R}_{\mathrm{wr}}$, abs-steps ${ }_{\mathrm{wr}}, \mathscr{I}_{\mathrm{wr}}$ meet decomposed secure-refinement requirements).

$$
\frac{\text { strong-low-bisim-mm } \mathscr{B} \quad \text { no-high-branching } \mathscr{B}}{\text { secure-refinement-decomp } \mathscr{B} \mathscr{R}_{\text {wr }} \mathscr{I}_{\text {wr }} \text { abs-steps }{ }_{\text {wr }}}
$$

Proof. Unfolding Definition 2.18, the obligations pertaining only to $\mathscr{R}_{\mathrm{wr}}$ and abs-steps $\mathrm{wr}_{\mathrm{wr}}$ are discharged by Lemma 5.12, Lemma 5.9, and Lemma 5.8. Pertaining to $\mathscr{I}_{\text {wr }}$ : Clearly $\mathscr{I}_{\text {wr }}$ is symmetric, and furthermore it is cg-consistent (Definition 2.6) because the actions over which $\mathscr{I}_{\text {wr }}$ must be closed modify only the shared memory, and $\mathscr{I}_{\text {wr }}$ places only restrictions on the program text and current location. The final obligation (regarding decomp-refinement-safe) is discharged by Lemma 5.14

From this it follows immediately via Theorem 2.20 that $\mathscr{R}_{\mathrm{wr}}$ with the help of $\mathscr{I}_{\mathrm{wr}}$ describes a confidentiality-preserving refinement for non-High-branching While programs:

Corollary 5.16 ( $\mathscr{R}_{\mathrm{wr}}$ is a secure refinement for non-High-branching programs).

$$
\frac{\text { strong-low-bisim-mm } \mathscr{B} \quad \text { no-high-branching } \mathscr{B}}{\text { secure-refinement } \mathscr{B} \mathscr{R}_{\text {wr }} \mathscr{I}_{\text {wr }}}
$$

Finally we prove that successful compilation produces a RISC program related by $\mathscr{R}_{\mathrm{wr}}$ to its input While program, when started with corresponding (same $m d s, m e m$ ) and reasonable (according to compiled-cmd-config-consistent) initial configurations:

Theorem 5.17 (Successful compilations are refinements in $\mathscr{R}_{\mathrm{wr}}$ ).

$$
\left(P C s, l^{\prime}, n l^{\prime}, C^{\prime}, \text { failed }\right)=\text { compile-cmd } C l n l c \quad \text { compile-cmd-input-reqs } C l n l c
$$

failed $=$ False $\quad$ compiled-cmd-config-consistent $C$ regs $m d s$ mem $\quad P=$ map fst $P C s$

$$
\left(\langle c, m d s, m e m\rangle_{\mathrm{w}},\langle((0, P), r e g s), m d s, m e m\rangle_{\mathrm{r}}\right) \in \mathscr{R}_{\mathrm{wr}}
$$

Proof. By induction on the structure of the While-language.

The compiler input and initial configuration conditions we impose allow us to have each of skip, $c m d$; $c m d$, if $\exp$ then $c m d$ else $c m d$ fi, while $\exp$ do $c m d$ od, $v:=\exp , \operatorname{lock}(k)$, and $\operatorname{unlock}(k)$ and their compiled output meet the guards of the introduction rules for the cases skip, seq, if_expr, while_expr, assign_expr, lock_acq, and lock_rel of $\mathscr{R}_{\mathrm{wr}}$ (described further in Appendix C) that we designed for them, respectively. 


\subsection{Proof of compositional noninterference preservation}

Going beyond the level of detail of our presentation in Sison \& Murray (2019), we now present the final few steps to obtain preservation of whole-system security for concurrent compositions of RISC threads when all are obtained via compilation by the wr-compiler Section 5.4.1). In addition to this, we obtain preservation of per-thread compositional security for each program thread compiled, and other properties that may be useful for their composition with RISC threads proved secure directly at the RISC level (Section 5.4.2).

\subsubsection{Whole-system security preservation}

To use the whole-system refinement theorem (Theorem 2.23), we are obliged to show that, in addition to establishing a secure-refinement (Definition 2.15, which we just showed in Section 5.3, the wr-compiler also preserves local-mode-compliance as demanded by compositional-refinement Definition 2.22). Then, as we noted in Section 2.5, there is no need for us to prove preservation of the non-compositional global-modes-compatibility condition - the whole-system refinement theorem takes care of that.

The local compliance preservation result follows from a property of the refinement relation, $\mathscr{R}_{\mathrm{wr}}$. Here, "respects-own-guarantees" is from Definition 2.11,

Lemma 5.18 (Each step from a RISC configuration in $\mathscr{R}_{\mathrm{wr}}$ respects its own guarantees).

$$
\frac{\left(\langle c, m d s, m e m\rangle_{\mathrm{w}},\langle((p c, P), \text { regs }), m d s, \text { mem }\rangle_{\mathrm{r}}\right) \in \mathscr{R}_{\mathrm{wr}}}{\text { respects-own-guarantees }(((p c, P), \text { regs }), m d s)}
$$

Proof. By induction on the structure of $\mathscr{R}_{\mathrm{wr}}$.

Knowing that the While command does not access lock-governed variables without holding the relevant lock (via the stability-checks asserted as part of compile-cmd-input-reqs by every relevant case of $\mathscr{R}_{\mathrm{wr}}$ ), we are obliged to show that the RISC instruction paired to it by $\mathscr{R}_{\text {wr }}$ similarly respects the guarantee modes implied by the locking discipline (as specified in Section 3.1. . We do so with a mixed Isar/"apply"-style proof that exercises the relevant cases of the RISC semantics, using lemmas about control flow under sequential composition (mentioned in Section 5.1, see also Appendix A). Propositions 3.8 and 3.9 also play a role in excluding certain cases from consideration.

Lemma 5.19 (Refinements in $\mathscr{R}_{\mathrm{wr}}$ ensure local mode compliance).

$$
\frac{\left(\langle c, m d s, m e m\rangle_{\mathrm{w}},\langle((p c, P), \text { regs }), m d s, m e m\rangle_{\mathrm{r}}\right) \in \mathscr{R}_{\mathrm{wr}}}{\text { local-mode-compliance }\langle((p c, P), \text { regs }), m d s, m e m\rangle_{\mathrm{r}}}
$$

Proof. Unfolding Definition 2.11, we must show that what was proved by Lemma 5.18 holds for every RISC configuration reachable from $\langle((p c, P), \text { regs }), m d s, m e m\rangle_{r}$.

First, we prove a lemma that establishes that every such reachable RISC configuration is also paired by $\mathscr{R}_{\mathrm{wr}}$ to some While configuration. Specifically, we prove that $\mathscr{R}_{\mathrm{wr}}$ is closed under a notion of "pairwise reachability under mode-permitted havoc", wherein:

1. Every one step by the RISC program is matched by either zero or one step by the While program, as specified by abs-steps ${ }_{w r}$ Section 5.3.2. 
2. Between each evaluation step, arbitrary changes are allowed to occur to the memory locations judged by the mode state to be writable (Definition 2.5).

Because all such RISC configurations reachable from the initial one are in $\mathscr{R}_{\mathrm{wr}}$, it then follows from Lemma 5.18 that they respect their own guarantees, as required.

We then initialise the compiler with an empty $C_{0}::$ CompRec that knows nothing about the register contents, and does not assume any variables to be stable:

Definition 5.20 (Empty compilation record $C_{0}$ ).

$$
\mathrm{C}_{0} \triangleq\left(\left(\lambda_{-} . \text {None }\right),(\emptyset, \emptyset)\right)
$$

With these definitions we have the desired consistency result:

Lemma 5.21 (Initial $\mathrm{C}_{0}, \mathrm{mds}_{0}$ are consistent with no-locks-held).

no-locks-held $m e m \Longrightarrow$ compiled-cmd-config-consistent $\mathrm{C}_{0}$ regs $\mathrm{mds}_{0}$ mem

Proof. This is straightforward by unfolding Definitions 5.7, 3.18, 3.19, and 5.20, also relying on the cleanliness conditions Proposition 3.5 and Proposition 3.7 on locking disciplines specified in Section 3.2.

We now have enough information to derive a whole-system security result, for concurrent RISC programs obtained by running the wr-compiler on any list " $c s$ " of secure While commands (one for each thread in the program). As we explained in Section 5.2, the CoverN wr-compiler's preservation of security is only for programs with no-high-branching Definition 5.5); furthermore, so that we can derive global compatibility for multiple of these programs run concurrently as threads (as per Section 3.4), we will impose no-locks-held (Definition 3.18) as an initial condition. Therefore, the security preservation theorem we choose to prove here demands that every thread of the input While program be com-secure ${ }_{\text {no-locks-held }}^{\text {no-high-branching }}$ (Definition 2.7, with additional requirements as specified). It then promises that the output program is sys-secure no-locks-held: :

Theorem 5.22 (Secure threads compiled by the wr-compiler form a secure system).

$$
\begin{aligned}
& \text { length } c m s_{r}=\text { length } c s \wedge \\
& \forall i<\text { length } c m s_{r} . \exists l n l P C s l^{\prime} n l^{\prime} C^{\prime} \text { regs. } \\
& \text { com-secure no-high-branching }\left(c s[i], \text { mds }_{0}\right) \wedge
\end{aligned}
$$

$\left(\forall m e m\right.$. no-locks-held $m e m \longrightarrow$ local-mode-compliance $\left.\left\langle c, \text { mds }_{0}, m e m\right\rangle_{\mathrm{w}}\right) \wedge$

$\left(P C s, l^{\prime}, n l^{\prime}, C^{\prime}\right.$, False $)=$ compile-cmd $C_{0} l n l c s[i] \wedge$ compile-cmd-input-reqs $C_{0} l n l c s[i] \wedge$ $\mathrm{cms}_{r}[i]=\left(((0\right.$, map fst $P C s)$, regs $\left.), \mathrm{mds}_{0}\right)$

$$
\text { sys-secure } \text { no-locks-held } \mathrm{cms}_{r}
$$

Proof. We invoke Theorem 2.23 , supplying:

- no-locks-held for the INIT parameter at both While and RISC level. 
- $\mathscr{B}_{\text {all }}, \mathscr{R}_{\text {wr }}, \mathscr{I}_{\text {wr }}$ to be respectively the witness bisimulation, refinement relation, and coupling invariant for all compiled threads, where we define $\mathscr{B}_{\text {all }}$ to be the arbitrary union of all strong low-bisimulations modulo modes that disallow high-branching:

$$
\mathscr{B}_{\text {all }} \triangleq \bigcup\{\mathscr{B} \mid \text { strong-low-bisim-mm } \mathscr{B} \wedge \text { no-high-branching } \mathscr{B}\}
$$

- $\mathrm{mds}_{0}$ to be the initial mode state for all While threads in $c s$.

The first thing we must prove is that the original program satisfies sound-mode-use Definition 2.10 when initialised with $\mathrm{mds}_{0}$ and no-locks-held; we have the local part from this theorem's local-mode-compliance assumption, and the global part from Lemma 3.20.

We then discharge the demands of compositional-refinement $\mathscr{B}_{\text {all }} \mathscr{R}_{\mathrm{wr}} \mathscr{I}_{\mathrm{wr}}$ (Definition 2.22) using Corollary 5.16, Lemma 5.19, and by unfolding Definition 5.13.

It only remains for us to show that the initial RISC-While and While-While configuration pairs of interest are captured respectively by $\mathscr{R}_{\mathrm{wr}}$ and $\mathscr{B}_{\text {all }}$. We obtain the former using this theorem's assumptions and Lemma 5.21 to discharge the guards of Theorem 5.17.

Finally, we use the assumption that the original program is com-secure no-locks-held $^{\text {no-hing }}$ and unfold Definition 2.7 to obtain that there exists some strong-low-bisim-mm $\mathscr{B}$ that enforces no-high-branching for every configuration pair with low-equal memories (modulo $\mathrm{mds}_{0}$ ) and no-locks-held initially; therefore, these state pairs must all be captured by $\mathscr{B}_{\text {all }}$.

\subsubsection{Per-thread compositional security preservation}

For system developers who may want to run programs compiled from While to RISC concurrently with other programs written directly in RISC, per-thread security preservation results may be useful. To compose the security proofs for those threads, direct RISC-level lemmas for the "sound-mode-use" side conditions of the compositionality theorem Theorem 2.8) will also be needed. We therefore present these as an alternative method to obtain compositional security results for RISC programs, applicable when only partially produced by compilation from While by the wr-compiler.

Given the facts we established in Section 5.3, we have straightforwardly that such programs' executions are captured by the bisimulation derived from $\mathscr{B}, \mathscr{R}_{\mathrm{wr}}, \mathscr{I}_{\mathrm{wr}}$, when started with reasonable initial configurations corresponding to those paired by $\mathscr{B}$ :

Lemma 5.23 (Programs witnessed by $\mathscr{B}$ are captured by $\mathscr{B}_{\mathrm{C}}$ of $\mathscr{B} \mathscr{R}_{\mathrm{wr}} \mathscr{I}_{\mathrm{wr}}$ once compiled).

$$
\begin{gathered}
\text { strong-low-bisim-mm } \mathscr{B} \quad\left(\left\langle c, m d s, \text { mem }_{1}\right\rangle_{\mathrm{w}},\left\langle c, m d s, \text { mem }_{2}\right\rangle_{\mathrm{w}}\right) \in \mathscr{B} \\
\left(P C s, l^{\prime}, n l^{\prime}, C^{\prime}, \text { failed }\right)=\text { compile-cmd } C l n l c \quad \text { compile-cmd-input-reqs } C l n l c \\
\text { failed }=\text { False } \quad \text { compiled-cmd-config-consistent } C \text { regs } m d s m^{2} \quad P=\text { map fst } P C s \\
\text { compiled-cmd-config-consistent } C \text { regs } m d s \text { mem }_{2}
\end{gathered}
$$

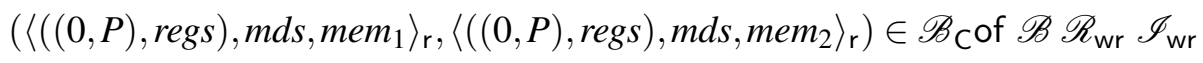

Proof. Straightforward from the definition of $\mathscr{B}_{C}$ of (Definition 2.16), using Theorem 5.17 to show membership of $\mathscr{R}_{\mathrm{wr}}$, and the definition of strong-low-bisim-mm (Definition 2.4 to show that the memories are low-equal modulo modes, as required by $\mathscr{B}_{\text {Cof. Finally, }}$ membership of $\mathscr{I}_{\mathrm{wr}}$ (Definition 5.13 follows from the fact that the paired configurations are at the same location (program counter 0 ) of the same program $P$. 
We are ready to state the per-thread security preservation result formally. Given an input While command that satisfies com-secure no-high-branching with $\mathrm{mds}_{0}$ initially, it promises that the RISC program output by the wr-compiler is com-secure $e_{\text {no-locks-held }}^{\text {pc-security }}$ wds 0 :

Theorem 5.24 (Preservation of per-thread confidentiality by the wr-compiler).

$$
\frac{\left(P C s, l^{\prime}, n l^{\prime}, C^{\prime}, \text { False }\right)=\begin{array}{cl}
\text { com-secure } \\
\text { no-locks-held }
\end{array}}{\text { compile-cmd } \mathrm{C}_{0} l n l c} \begin{aligned}
& \text { compile-cmd-input-reqs } \mathrm{C}_{0} l n l c \\
& \text { com-secure }
\end{aligned}
$$

Proof. We are given by com-secure ${ }_{\text {no-locks-held }}^{\text {no-high-branching }}$ Definition 2.7 that for low-equal starting configurations (modulo modes) of $c$ with no locks held, there exists some witness $\mathscr{B}$ satisfying both strong-low-bisim-mm and no-high-branching.

From this and Lemma 5.23 we have that the output program's corresponding execution is captured by a RISC semantics-level relation $\mathscr{B}_{C}$ of $\mathscr{B} \mathscr{R}_{\text {wr }} \mathscr{I}_{\text {wr }}$ derived from this $\mathscr{B}$, with Lemma 5.21 discharging the compiled-cmd-config-consistent requirements.

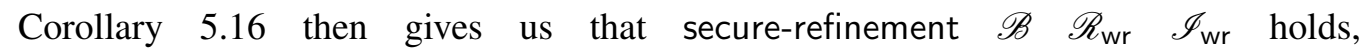
and from this and strong-low-bisim-mm $\mathscr{B}$ using Theorem 2.17 we have strong-low-bisim-mm $\left(\mathscr{B}\right.$ Cof $\left.\mathscr{B} \mathscr{R}_{\text {wr }} \mathscr{I}_{\text {wr }}\right)$. This is enough to show com-secure no-locks-held for the RISC program, by Definition 2.7, as Section 5.3.3 noted, pc-security (Definition 5.6) is immediate from the definition of $\mathscr{I}_{\mathrm{wr}}$.

To prove a whole-system security result at the RISC level for the compiled program, we must also prove sound-mode-use (Definition 2.10). To that end, we prove a local and global result for RISC programs output by the wr-compiler when given a secure While program. The former follows from the local compliance result in the preceding section:

Lemma 5.25 (Threads compiled by the wr-compiler obey local compliance).

$$
\begin{gathered}
\left(P C s, l^{\prime}, n l^{\prime}, C^{\prime}, \text { False }\right)=\text { compile-cmd } \mathrm{C}_{0} l n l c \quad \text { compile-cmd-input-reqs } \mathrm{C}_{0} l n l c \\
\text { no-locks-held mem }
\end{gathered}
$$

Proof. We use Theorem 5.17 and Lemma 5.21 to obtain membership in $\mathscr{R}_{\mathrm{wr}}$, which then allows us to use Lemma 5.19.

Then we prove invariance of global modes compatibility (as in Section 3.3 for compiled RISC programs, due to RISC's identical semantics to While regarding locking and modes:

Lemma 5.26 (Initialising RISC with no-locks-held, mds 0 ensures global compatibility).

$$
\frac{\text { no-locks-held } m e m \quad \forall(((p c, P), r e g s), m d s) \in \text { set } c m s_{r} . m d s=\mathrm{mds}_{0}}{\text { global-modes-compatibility }\left(\mathrm{cms}_{r}, \mathrm{mem}\right)}
$$

Proof. We firstly prove versions of Lemma 3.15, Lemma 3.16, and Theorem 3.17 for RISC, following exactly the same reasoning as we did in Section 3.3 for While. This is because the RISC instructions LockAcq $k$ and $\operatorname{LockRel} k$ are (like $\operatorname{lock}(k)$ and $\operatorname{unlock}(k)$ in While) 
the only ones in their language that modify mode state, and their semantics regarding mode state and lock memory are identical to those of the $\operatorname{lock}(k)$ and $\operatorname{unlock}(k)$ commands. The present result then follows for the same reason that Lemma 3.20 did for While.

With this result, it is now possible to invoke Theorem 2.8 to compose RISC-level perthread security and mode compliance, whether they were obtained via the wr-compiler (using Theorem 5.24 and Lemma 5.25, respectively), or proved directly at RISC level.

We remark that, for programs wholly compiled by the wr-compiler, Theorem 5.22 can be subsumed by a whole-system preservation result that no longer demands local-mode-compliance for each thread, due to our ability to obtain it directly at RISC level:

Theorem 5.27 (Secure threads compiled by the wr-compiler form a secure system).

$$
\begin{aligned}
& \forall i<\text { length } c m s_{r} . \exists c l n l P C s l^{\prime} n l^{\prime} C^{\prime} \text { regs. } \\
& \text { com-secure } \text { no-high-branching }_{\text {no-lockseld }}\left(c, \text { mds }_{0}\right) \wedge \\
& \left(P C s, l^{\prime}, n l^{\prime}, C^{\prime}, \text { False }\right)=\text { compile-cmd } C_{0} l n l c \wedge \text { compile-cmd-input-reqs } C_{0} l n l c \wedge \\
& c m s_{r}[i]=\left(((0, \text { map fst } P C s), \text { regs }), \text { mds }_{0}\right)
\end{aligned}
$$

$$
\text { sys-secure } \text { no-locks-held } \mathrm{cms}_{r}
$$

Proof. By Theorem 2.8 and unfolding Definition 2.10, we are required to prove security and local mode compliance for every thread of the compiled RISC program, and global modes compatibility between them all as a whole, assuming no-locks-held and using mds $\mathrm{s}_{0}$ initially. These requirements are immediate using Theorem 5.24, Lemma 5.25, and Lemma 5.26.

\section{Case study: Cross Domain Desktop Compositor input handler}

This section presents-as the main case study for the COVERN wr-compiler-a mixedsensitivity concurrent program whose source-level noninterference properties are preserved by verified secure compilation down to an assembly-level model.

The Cross Domain Desktop Compositor (CDDC) of Beaumont et al. (2016) is a desktop device that gives trusted users the option of replacing multiple monitors, keyboards, and mice with a single multi-level secure user interface (via a single monitor, keyboard, and mouse, as depicted in Figure 5a when using several desktop computers simultaneously.

Here we present as case study a program (replacing customised hardware) that handles the incoming mouse and keyboard inputs to the CDDC. This program has served as a particularly good case study, because it features both of the characteristics for which proving information-flow security is this work's main focus:

- Concurrency-here, between software components whose execution is interleaved (by the seL4 operating-system microkernel (Klein et al., 2014), and that interact via shared memory.

- Mixed-sensitivity reuse-here, of system resources (notably the input devices) and memory locations, for input whose sensitivity level can be different at different times. 


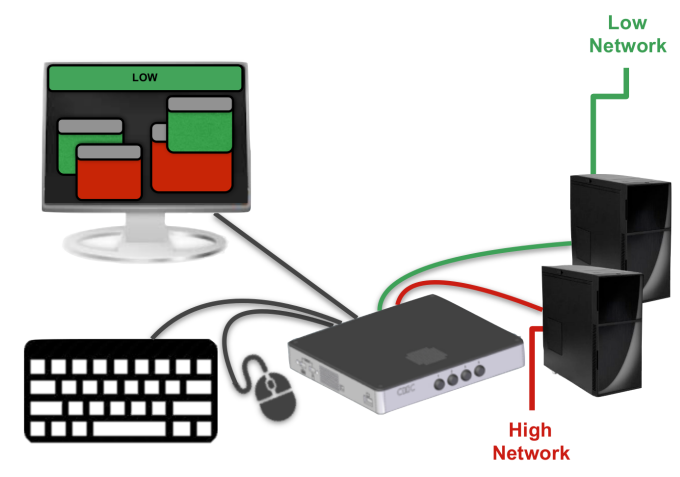

(a) CDDC hardware use-case setup.

The bar painted at the top of the screen indicates the computer set to receive all keyboard events. Mouse events are delivered to the owner of the topmost window underneath the mouse cursor.

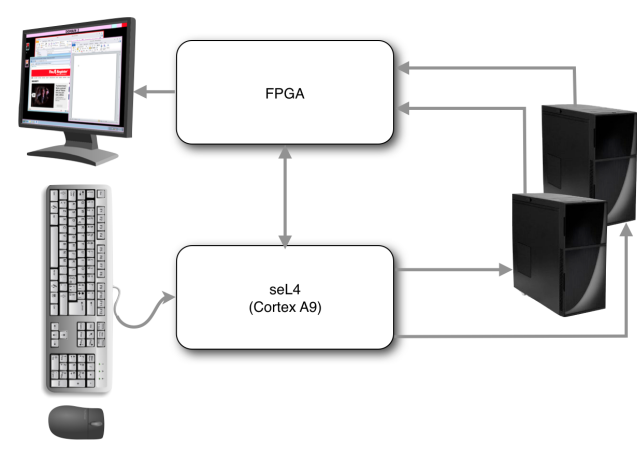

(b) CDDC hardware architecture.

The HID switch-implemented in software on top of seL4-runs on an ARM Cortex A9 core, and operates a compositor device implemented (as in Beaumont et al. (2016)) using an FPGA.

Figure 5: Functional schematics for Cross Domain Desktop Compositor hardware. Reproduced from Murray et al. (2018).

By exercising the COVERN wr-compiler on a While model of this case study, we show this compiler verification-based approach to be feasible for obtaining the preservation of noninterference properties proved at While level, straightforwardly and for little extra effort, down to a RISC model of the program.

The section will proceed as follows. Following an overview in Section 6.1 of the main characteristics of the case study, Section 6.2 presents the formal security properties proved about its While model - as our focus is its compilation, further details on this model and the proof techniques used to prove these properties at While level are left to Sison (2020). Section 6.3 then presents the formal preservation of security properties down to a RISC model, obtained from running the verified wr-compiler of Section 5 on the While model.

\subsection{Overview of the case study}

The case study is a software implementation of the human interface device (HID) switch in the CDDC (see Figure 5b). In short, this part of the CDDC is responsible for determining the destination of all HID input (keyboard and mouse device) events, and ensuring that the user remains informed of that destination (by operating a video compositor device, which renders display elements for that purpose on a shared monitor, as depicted in Figure 5a.

\subsubsection{Information-flow security}

The HID switch's responsibilities are security critical, as the CDDC is intended to provide an interface to multiple desktop computers belonging to different security domains; hence, the user of the CDDC is expected to choose the sensitivity of the data they input, based on the computer to which they expect it to be delivered. Furthermore, part of the CDDC's functionality is to allow users to choose which computer they are interacting with, by clicking on (accordingly responsive) display elements using the mouse. Thus, the desired information- 


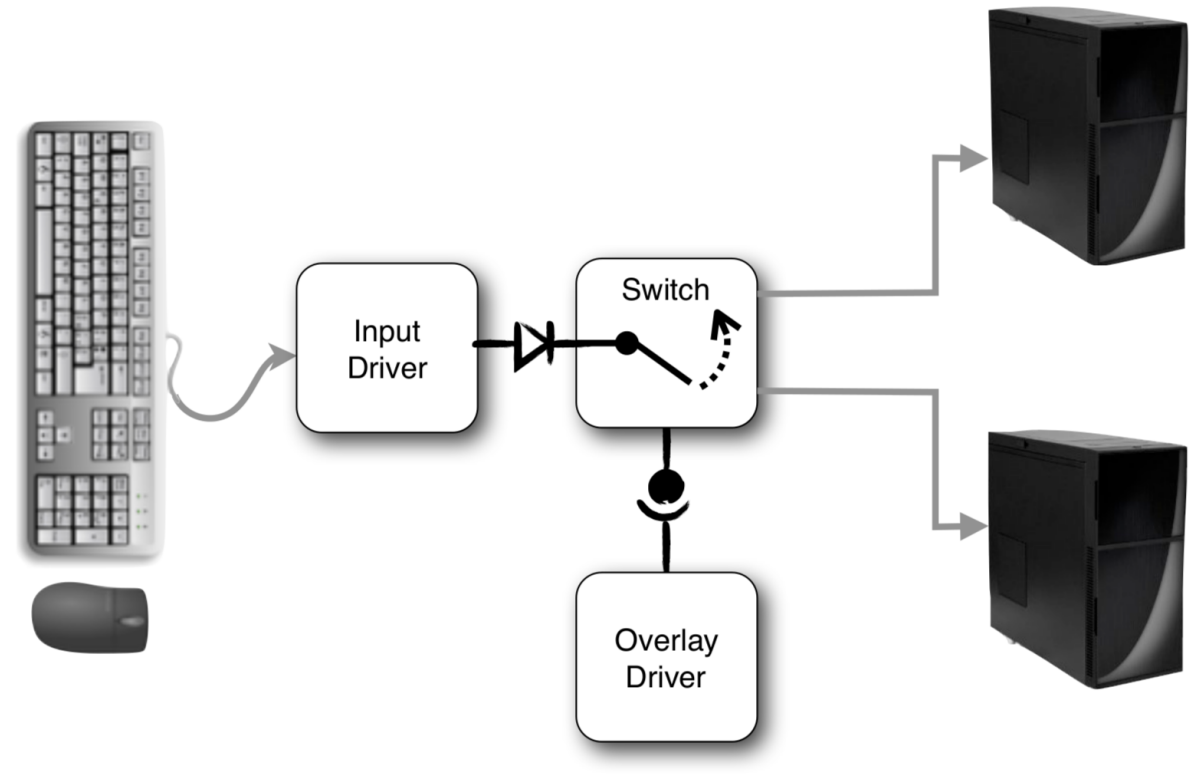

Figure 6: Functional schematic of seL4 component architecture for CDDC HID switch. Reproduced from Murray et al. (2018).

flow security property for the HID switch is that, in providing this functionality, it never delivers inputs to a destination contrary to the user's expectations.

We simplify analysis to the classic High $\not \rightarrow$ Low security policy over the basic two-point \{High, Low security lattice, and model the HID switch to service only two potential destination computers.$^{7}$ One computer is designated as belonging to the High security domain, and is the only legitimate destination for High-sensitivity input events; the other is designated as belonging to the Low security domain. The hardware and connections that the SWITCH component uses to forward events to these computers are modelled as shared variables classified statically: one High, the other Low (as depicted in Figure 7b). The attacker is then considered to be an entity that can read at any time from the Low-classified one.

\subsubsection{Shared-variable concurrency}

The software implementation (replacing the original FPGA-based implementation (Beaumont et al. 2016)) of the CDDC's HID switch is a system of software components written in C, that all run in user mode on top of the seL4 microkernel (Klein et al., 2014).

Here, we have abstracted from the seL4-based C implementation's details, to model in the While language the basic functionality of its three main software components (as depicted in Figure 6) as a shared-variable concurrent program of three threads:

- The INPUT driver is responsible for taking events from input-device interfaces and placing them on an input-event buffer for consumption by the SWITCH (Figure 7a).

\footnotetext{
${ }^{7}$ Aside from presenting a more minimal case study, any verification for an arbitrary security lattice can be reduced to multiple applications of verification to the basic High $\not \rightarrow$ Low policy, with the locations reclassified appropriately. Furthermore, the design of the CDDC's HID switch program is symmetrical for each user.
} 


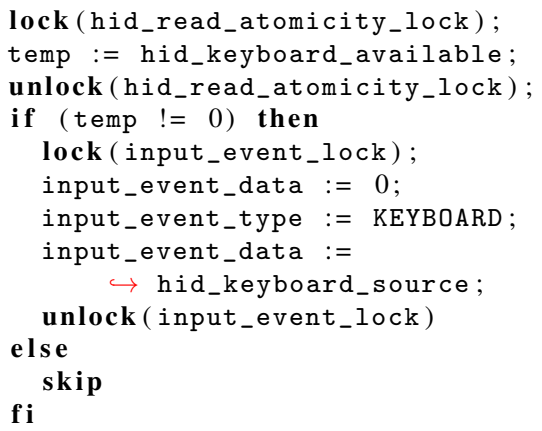

(a) Receipt from input device by INPUT driver. The hid_keyboard_source variable is valuedependently classified by the value of its sole control variable, indicated_domain (modelling trusted user input to the keyboard).

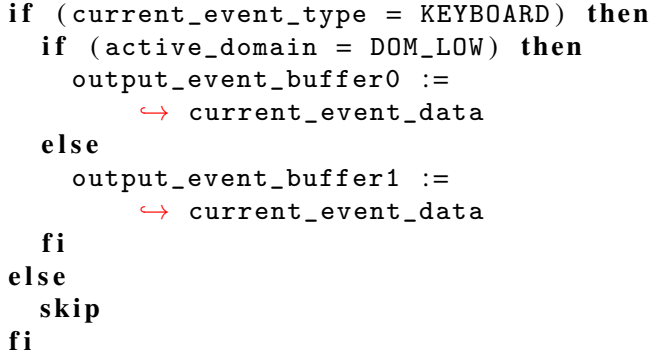

(b) Delivery to output device by SwITCH.

The output-event buffers 0 and 1 are statically classified Low and High respectively (modelling an attacker-controlled computer that receives all data written to buffer 0 ).

Figure 7: Examples of external device interactions by the CDDC HID switch, as modelled in While-here, for the keyboard events. The full model is in the Isabelle/HOL supplement.

- The SwITCH is responsible for inspecting all input events on the buffer from the INPUT driver, querying the compositor device (as modelled in Figure 8a) and OVERLAY driver to determine if any constitute a user-directed change to the destination of subsequent events, and if so, updating the compositor device to display that change (as modelled in Figure 8b). Finally, it is responsible for delivering all events to their destination computer via the appropriate output-device interface (Figure 7b).

- The OVERLAY driver is responsible for servicing remote procedure calls (RPCs, made by the SWITCH) that query a subset of the compositor-device interface, regarding the position of certain mouse-clickable elements the compositor is rendering as part of a visual overlay on the trusted user's video monitor. (As no mixed-sensitivity reuse occurs in this part of the model, we leave its details to the Isabelle/HOL supplement.)

The device interfaces, shared buffers (for input events and RPC mechanisms), and local variables used by each component are all modelled as program variables in shared memory. Consequently in the While model, mutex locks are used to model all synchronisation and restriction of concurrent access by the components to those variables.

So that we do not need to add separate While semantics for interacting with private as opposed to shared memory, we model thread-private memory as shared program variables protected by a permanently held lock acquired at initialisation time (e.g. as in Figure 9a). We consider this to be a stand-in for the memory isolation properties established by the underlying operating system between the program threads that it hosts.

\subsubsection{Mixed-sensitivity reuse}

Inherently to the CDDC's role as a multi-level secure user interface, its HID switch receives data of differing sensitivity levels (at different times) from a single set of input device mem- 


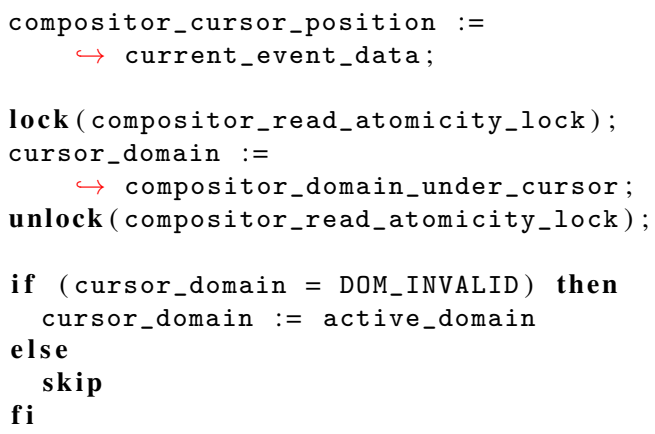

(a) Querying the compositor to determine the topmost domain under the mouse cursor.

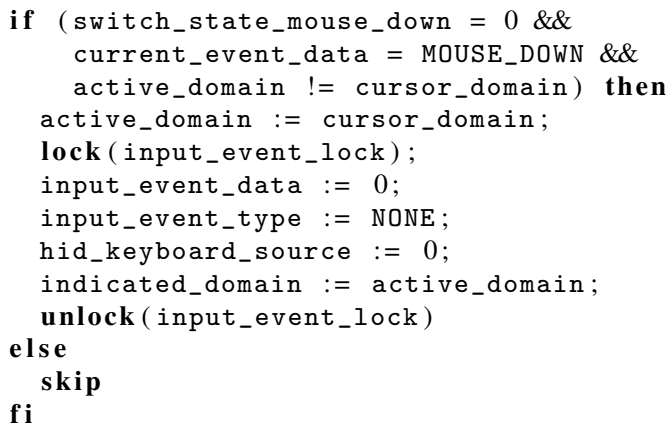

(b) Instructing the compositor to indicate a change to the active domain.

Figure 8: Excerpts of the SWITCH component interfacing with the compositor device.

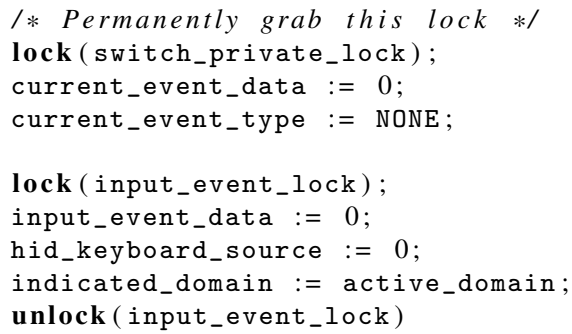

(a) Initialising private variables, input-event buffer, and compositor-indicated domain, to an arbitrary initial value for active_domain. Zeroing the data fields prevents leaking any Highsensitivity data they might initially contain.

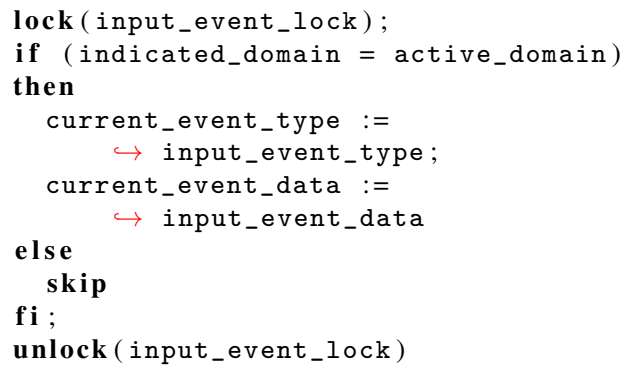

(b) Copying from the input-event buffer to private variables. The security analysis shows that repeating the previous event is a safe course of action when the environment misbehaves by violating indicated_domain = active_domain.

Figure 9: Excerpts of the SWITCH component interacting with the input-event buffer.

ory locations (e.g. as modelled in Figure 7a, for the keyboard events), rather than from those of distinct device sets for each sensitivity level.

Furthermore, the HID switch propagates all input event data (regardless of sensitivity) through a single set of memory locations (the input-event buffer and SWITCH-internal copies of its contents, as modelled in Figure 9), rather than duplicating those memory locations for each security domain. Consequently in the While model, all of these memory locations that are subject to mixed-sensitivity reuse are assigned value-dependent classifications, reflecting the trusted user's expectation of the sensitivity level of the data they contain:

- To model a user that we trust to type sensitive information into the keyboard only when the compositor device indicates the High domain computer is active (i.e. set to receive all keyboard events), we have the INPUT driver draw keyboard events from a shared variable named hid_keyboard_source (as depicted in Figure 7a) that has classification dependent on a control variable indicated_domain modelling the rel- 
evant state of the compositor (here, DOM_HIGH is a designated constant):

$$
\begin{cases}\text { High, } & \text { if indicated_domain }=\text { DOM_HIGH } \\ \text { Low, } & \text { otherwise. }\end{cases}
$$

- In contrast, as clicking on composited user interface elements has the potential ability to change the future indicated_domain (which, as a control variable, is never allowed to receive any High-sensitivity data), the model trusts the user not to encode sensitive information into the mouse input in any way. Thus, the INPUT driver always draws mouse events from a statically Low-classified shared variable.

Consequently, as the data portion input_event_data of the input-event buffer ${ }^{8}$ between the INPUT driver and SWITCH may carry either keyboard data of valuedependent sensitivity or Low-sensitivity mouse data, we assign it a classification dependent on the values of both its control portion and the indicated_domain:

$$
\begin{cases}\text { High, } & \text { if input_event_type }=\text { KEYBOARD } \wedge \text { indicated_domain }=\text { DOM_HIGH } \\ \text { Low }, & \text { otherwise. }\end{cases}
$$

- Finally, we model the seL4-based SwITCH component's copying of the event from the buffer into its own local variables, giving its data portion a classification dependent on its own private view of the currently active domain (modelled as active_domain):

$$
\begin{cases}\text { High, } & \text { if current_event_type }=\text { KEYBOARD } \wedge \text { active_domain }=\text { DOM_HIGH } \\ \text { Low, } & \text { otherwise. }\end{cases}
$$

To ensure active_domain remains authoritative with what is composited by the CDDC into the display, in the While model the SWITCH initialises indicated_domain to match the initial value of active_domain (as depicted in Figure 9a), updates it whenever active_domain changes (as depicted in Figure 8b), and checks at runtime that active_domain = indicated_domain when copying data from the buffer to its own private variables (as depicted in Figure 9b).

The CVDNI properties' (1) value dependence on control variables, (2) quantification over all initial values for the control variables and (3) assumptions of environmental havoc on write-unprotected shared variables between evaluation steps (Definition 2.6 then ensure noninterference between High inputs and Low-classified sinks, regardless of the initial and dynamically changing sensitivity of all such locations subject to mixed-sensitivity reuse.

\subsection{CVDNI properties of the While model to be preserved}

This section will now give a brief formal exposition of the security properties of the CDDC HID switch's While-language model that our compiler will preserve down to RISC.

As the per-thread proof techniques for While that we used for the case study are outside the scope of this paper, we note only that they consist of an adaptation to mutex locks by

\footnotetext{
${ }^{8}$ We model in While only a single-place buffer, which could easily be extended to a buffer of arbitrary size by duplicating the same basic pattern of access, classification, and lock-protection, for multiple places.
} 
Sison (2020) of a security type system and local mode compliance check developed by Murray et al. (2016b c). Nevertheless, we have provided their full formalisation in our Isabelle/HOL supplement, and we refer the reader to these prior works on their design, and particularly to Sison (2020) for further discussion on their application to this case study.

In short, from applying local type checks on the While-language commands for each of the three software components (INPUT, SWITCH, and OVERLAY) to obtain per-thread security (com-secure, Definition 2.7) and modes compliance (local-mode-compliance, Definition 2.11, we have from Theorem 2.8 that the concurrent program of all three components satisfies the whole-system security property (sys-secure, Definition 2.9) as instantiated to specify that no locks are held initially.

So that we can use the approach we gave in Section 3 to obtain the global modes compatibility part of the sound-mode-use side-condition (Definition 2.10), we specify no-locks-held (Definition 3.18) as the INIT requirement on memory, and use the initial mode state mds 0 (Definition 3.19) for all of the components in the system.

This no-locks-held predicate and $\mathrm{mds}_{0}$ are both defined relative to a lock interpretation parameter that we supply (as required by Section 3.1) for the CDDC model. The locks in the CDDC model fall under the following categories:

- The locks coordinating inter-component interactions grant exclusive read-write access to the shared variables they govern.

- There are also locks granting the SWITCH and INPUT components exclusive readwrite access to a set of "private" variables each, for internal use. The components acquire these prior to entering their main loop, and never release them.

- Finally the model uses read-atomicity locks - a practice introduced in Section 5.1.1 These grant exclusive write access to shared variables used to model hardware interfaces, to make explicit an assumption (normally implicit in the atomicity of expression evaluation in the While language) that these variables will not have their value changed by the environment during a simple assignment from those variables.

Note that these read-atomicity locks are not needed to prove confidentiality for the While model, but rather we add them to satisfy the requirements demanded by the wr-compiler so that it can preserve confidentiality (via small-step semantic preservation) down to the RISC model.

The While-language proof techniques we apply to each thread of the program yield com-secure ${ }^{\text {no-high-branching, }}$ a stronger version of the per-thread CVDNI property that enforces no-high-branching (Definition 5.5). Furthermore, we have trivially from the definition of com-secure (Definition 2.7) that if a program is secure without imposing any initial conditions, then it remains secure if we impose any INIT parameter arbitrarily.

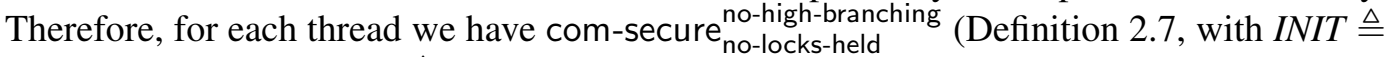
no-locks-held and EXTRA $\triangleq$ no-high-branching):

Lemmas 6.1 (Per-thread confidentiality results for CDDC While model).

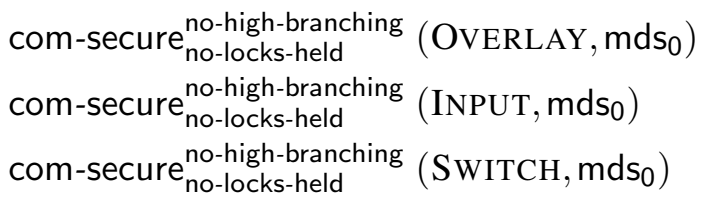


From this and Theorem 2.8, using local compliance checks and Lemma 3.20 to discharge the sound-mode-use (Definition 2.10) side condition, we have a whole-system confidentiality theorem for the system of all three components running concurrently:

Theorem 6.2 (Whole-system confidentiality result for the CDDC While model).

$$
\text { sys-secure } \text { no-locks-held }\left[(\text { OVERLAY, mds } 0),\left(\text { INPUT }, \text { mds }_{0}\right),\left(\mathrm{SWITCH}_{\mathrm{mds}}\right)\right]
$$

\subsection{Confidentiality-preserving compilation to RISC model}

We now turn to applying the COVERN wr-compiler of Section 5 to our While-language model of the CDDC's HID switch; we then have automatically that it preserves the security properties presented in Section 6.2 down to the compiler's RISC-language output.

The wr-compiler is executable in the Isabelle proof assistant. Using Isabelle's eval tactic, we execute the wr-compiler's main function, compile-cmd (whose implementation was described in Section 5.1 on the While-language models for all three of the CDDC's INPUT driver, SWITCH, and OVERLAY driver components, to obtain their RISC-language compilations. (Recall from Section 5.1 that we obtain the RISC text trivially as the map fst of the CompRec-annotated RISC program, which is the fst output of compile-cmd.)

Definition 6.3 (RISC-language program texts of CDDC model's components).

$$
\begin{aligned}
\text { OVERLAY }_{\mathrm{RISC}} & \triangleq \text { map fst }\left(\text { fst }\left(\text { compile-cmd } \mathrm{C}_{0} \text { None } 0 \text { OvERLAY }\right)\right) \\
\mathrm{INPUT}_{\mathrm{RISC}} & \triangleq \text { map fst }\left(\text { fst }\left(\text { compile-cmd } \mathrm{C}_{0} \text { None } 0 \text { INPUT }\right)\right) \\
\mathrm{SWITCH}_{\mathrm{RISC}} & \triangleq \text { map fst }\left(\text { fst }\left(\text { compile-cmd } \mathrm{C}_{0} \text { None } 0 \text { SWITCH }\right)\right)
\end{aligned}
$$

Our approach to obtain per-thread confidentiality for each of these RISC texts will be to use the theorem of its preservation by the wr-compiler (Theorem 5.24). Recall, this was:

Theorem 5.24 (Preservation of per-thread confidentiality by the wr-compiler).

$$
\frac{\left(P C s, l^{\prime}, n l^{\prime}, C^{\prime}, \text { False }\right)=\begin{array}{cl}
\text { com-secure } \\
\text { no-locks-held }
\end{array}}{\text { compile-cmd } \mathrm{C}_{0} l n l c} \begin{aligned}
& \text { compile-cmd-input-reqs } \mathrm{C}_{0} l n l c \\
& \text { com-secure }
\end{aligned}
$$

Then, for compile-cmd to execute successfully (i.e. to return failed $=$ False), the model must pass the stability-checks discussed in Section 5.1. All three of OVERLAY, INPUT, and SWITCH pass the checks (1) because they use locks to protect the atomicity of reads from (otherwise unstable) variables used to model hardware interfaces, and (2) as a consequence of having passed the local security and mode compliance checks mentioned in Section 6.2.

We are now in a position to prove a whole-system confidentiality result for the compiled RISC model—here, with each thread's register bank initialised to zero: regs $0 \triangleq\left(\lambda_{-} .0\right)$.

Theorem 6.4 (Whole-system confidentiality result for the CDDC RISC model).

$$
\begin{aligned}
\text { sys-secure }_{\text {no-locks-held }} & {\left[\left(\left(\left(0, \text { OVERLAY }_{\text {RISC }}\right), \text { regs }_{0}\right), \text { mds }_{0}\right),\right.} \\
& \left(\left(\left(0, \text { INPUT }_{\text {RISC }}\right), \text { regs }_{0}\right), \text { mds }_{0}\right), \\
& \left.\left(\left(\left(0, \text { SWITCH }_{\text {RISC }}\right), \text { regs }_{0}\right), \text { mds }_{0}\right)\right]
\end{aligned}
$$


Proof. A few approaches are available; we obtained formal proofs of this theorem in Isabelle/HOL using all three of the following alternatives (unfolding Definition 6.3):

Option 1. Use either of Theorem 5.22 or Theorem 5.27, both of which established whole-system security for RISC outputs of the wr-compiler when executed on com-secure no-high-branching While programs (which we have here from Lemmas 6.1). This is the easiest option to take for programs that are already verified in the While language, and then compiled successfully to RISC by the wr-compiler. It is possible to take here because

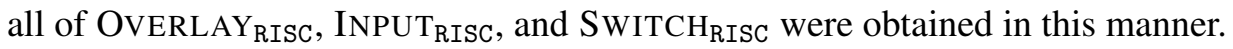

Option 2. Use Theorem 5.24 and Lemma 5.25 to obtain com-secure no-locks-held $_{\text {pcestity }}$ and local-mode-compliance (resp.) for each of OVERLAY RISC $_{\text {, INPUT }}$ RISC, and SWITCH RISC,

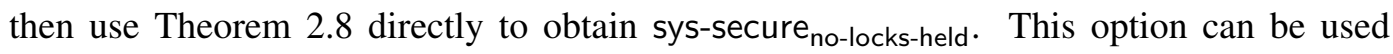
for systems where some of the threads are written directly in RISC; for such threads, com-secure ${ }_{\text {no-locks-held }}^{\text {pc-security }}$ and local-mode-compliance would need to be proved directly at RISC level. However, Lemma 5.26 still discharges the global-modes-compatibility requirement for RISC, provided all threads are initialised with $\mathrm{mds}_{0}$ and no-locks-held.

Option 3. Use Theorem 2.23 directly. This option can be used for systems where all the RISC threads are secure refinements (according to Definition 2.15) of the threads of some While program that satisfied sound-mode-use with no-locks-held initially, but some were obtained by other means than the wr-compiler (i.e. not all via the refinement $\mathscr{R}_{\mathrm{wr}}$ ).

\section{Related work}

First in Section 7.1, we describe other recent and related works that address concerns of noninterference proof compositionality in a concurrent setting (of the kind we tackled in Section 3). The remaining sections focus on related works on verified compilation: The works in Section 7.2 and Section 7.3, like ours, focus on compilation preserving a form of noninterference. In Section 7.4 we describe our work's relationship with varieties of robust property preservation, and other compilation verification efforts in Section 7.5.

\subsection{Compositionality of concurrent noninterference proofs}

Alternative approaches exist to establishing the non-compositional global modes compatibility condition we proved as invariant to concurrent While executions in Section 3 . For the precursor (non-value-dependent) notion of concurrent noninterference to CVDNI, Mantel et al. (2011) originally proposed that such a condition be met by a non-compositional may happen in parallel analysis (e.g. Masticola \& Ryder (1993)). Then, instead of demanding the explicit declaration of the sorts of guarantees implied by locking discipline (as we do), Mantel et al. (2015) proposed automating their inference and proof of the compatibility condition using a reachability analysis making use of dynamic pushdown networks. We leave adapting and implementing such an approach for our CVDNI setting to future work.

We note also that, like the CVDNI theory and our work of Section 3 , recent work by Frumin et al. (2021) concerns compositionality of machine-checked proof efforts for noninterference in a concurrent setting that are obtained potentially via a variety of proof techniques. They model more fine-grained synchronisation than we do here, via atomic compare-andswap operations that can be used to implement mutex locking primitives. However, they 
do not study compilation as a means of preserving such proofs, which is the focus of our work here. We believe that the CVDNI refinement notions we presented could support certain cases of compilation between different synchronisation primitives, provided only new thread-private state is needed (like the registers in RISC), and the shared variable interactions can be proved as preserved. For example, we expect mutex locking primitives (with slightly different semantics to ours here) could feasibly be refined to a compare-and-swap-based implementation in this way-this we also leave to future work.

\subsection{Noninterference-preserving compilation}

Tedesco et al. (2016) present a type-directed compilation scheme that preserves a faultresilient noninterference property. The compilation scheme of our wr-compiler was inspired by theirs. Like our com-secure CVDNI security property that wr-compiler preserves, Tedesco et al.'s security property is also strong bisimulation-based (Sabelfeld \& Sands, 2000). But where our property accounts (via mode states) for controlled interference by other threads, theirs instead quantifies over all possible interference by the environment with the memory contents. While this simplifies their task of proving that their security property is preserved under compilation-as it need not require the compiler to preserve the contents of memory-it means their security property cannot capture value-dependent noninterference. In contrast, our wr-compiler must obey our secure-refinement notion's requirement that memory contents are preserved.

The line of work most relevant to ours is that which was conducted (concurrently) by Barthe et al. (2020), wherein they achieved the remarkable result of proving that a modification of the CompCert C compiler (Leroy, 2009) preserves the cryptographic constant-time class of noninterference (2-safety) properties. Their proof approach was to use various notions of constant-time simulation (CT-simulation) first presented by Barthe et al. (2018), originally intended for application to the Jasmin compiler (Almeida et al., 2017$)$. Although not targeting programs with concurrency or mixed-sensitivity reuse (as our work does), CT-simulation shares in common with the refinement notions used by this paper that it in essence rests on a simulation diagram that is cube-shaped, as it must preserve a 2-safety hyperproperty. We submit that Barthe et al. (2020) broadly validates the argument we made in Sison \& Murray (2019), that decomposing such cube-shaped diagrams into square-shaped ones is what will make them feasible to apply to the verification of fully fledged compilers like CompCert-noting that they described the only compilation pass they proved with their non-decomposed, cube-shaped diagram as "not especially pleasant because the diagrams are difficult to exploit" (Barthe et al., 2020).

Note that the refinement theory of Barthe et al. (2018, 2020) preserves security via refinement phrased in terms of forward simulation (Leroy, 2009) - that is, each step of the abstract program must be simulated by the target program. In contrast, our theory presented here is instead targeted towards preserving refinement via backward simulations. 9 in which each step of the concrete (compiled) program must be simulated by the abstract program. This difference arises because in our setting we need to account for leakage that might occur

\footnotetext{
${ }^{9}$ Again, as commonly referred to in the compiler verification literature from Leroy (2009) onwards. This is not to be confused with the "backward simulations" of concurrency verification (Lynch \& Vaandrager. 1996) and data refinement (de Roever \& Engelhardt, 1998, Cavalcanti \& Naumann 2002), where the refined program instead simulates the original, and where simulation proceeds from the end of the program back to the beginning.
} 
and be visible only in intermediate states. In their setting, in contrast, leakage that occurs in intermediate states remains visible forever in the concrete program semantics via a leakage trace. It remains unclear whether we could have adopted a similar approach in our work, thereby enabling a (simpler) forward simulation argument. In particular, it is not clear what the semantics of leakage traces should be for a language that supports both value-dependent classification and shared-memory concurrency as ours does.

\subsection{Concurrency-compositional noninterference-preserving compilation}

Neither of the above consider per-thread compositional compilation of concurrent, shared memory programs, nor value-dependent noninterference policies - the focus of our theory and compiler. Barthe et al. $(2010$, 2007a) however did aim to preserve noninterference of multithreaded programs by compilation, extending a prior (security) type-preserving compilation approach (Barthe et al., 2004, 2007b). Their noninterference property however was termination- and timing-insensitive, so preventing internal timing leaks relied on the scheduler disallowing certain interleavings between threads. Also, their type-preservation argument was derived from a big-step semantics preservation property for their compiler. Here we instead rely on preservation of a small-step semantics (specifically memory contents), which is necessary for us to preserve value-dependent security under compilation, as well as to avoid imposing non-standard requirements on the scheduler.

\subsection{Robust property preservation}

Other recent works have improved on fully abstract compilation (surveyed by Patrignani et al. (2019)) by mapping out the spectrum (Abate et al., 2019) or developing specific forms (Patrignani \& Garg, 2019) of robust property preservation, concerned with robustness of source program (hyper)properties to concrete adversarial contexts. Like Tedesco et al. (2016), these works differ from ours in quantifying over a wider range of hostile interference. They also focus prominently on changes to data types, which we do not support. Thus, as a 2-safety hyperproperty quantifying over a lesser range of interference, we expect CVDNI-preservation to be implied by R2HSP (robust 2-hypersafety preservation), but do not expect it to imply any other secure compilation criterion on Abate et al.'s spectrum.

While recently Patrignani \& Garg (2019) instantiated their robustly safe compilation for shared-memory fork-join concurrent programs, it only preserves (1-)safety properties. Previously however, Patrignani \& Garg (2017) proved their trace-preserving compilation preserves $k$-safety hyperproperties (Clarkson \& Schneider, 2010), including noninterference properties. However, it disallows the removal or addition of trace entries, which would be necessary to change the passage of time as seen in the observable trace events. Thus it excludes the sorts of changes to pacing carried out by our compiler (regulated by abs-steps) and studied as optimisations by the two other works (Tedesco et al., 2016, Barthe et al. 2020) on timing-sensitive security-preserving compilation mentioned above.

\subsection{Compiler verification in general}

Finally, there has been much work on large-scale verified compilation (Leroy, 2009, $\overline{\mathrm{Ku}}$ mar et al. 2014) some of which has also treated compilation of shared-memory concurrent 
programs (Lochbihler, 2018) including taking weak-memory consistency into account (Podkopaev et al. 2019). Our work here does not consider the effects of weak-memory models. In particular, such models are often defined axiomatically rather than operationally. Our notion of secure refinement and our decomposition principle (Definitions 2.15 and 2.18 , respectively) are defined assuming an operational semantics for the source and target languages.

Our work differs to prior work on verified concurrent compilation, in that it formalises and proves a compiler's ability to use information about the application's locking protocol, both to exclude unsafe access to shared variables, and conversely to know when it is safe to allow optimisations on shared variables that would typically be excluded.

\section{Conclusion}

To our knowledge, we have presented the first mechanised verification that a compiler preserves concurrent, value-dependent noninterference. To this end, we provided a general decomposition principle for compositional, secure refinement. Although our compiler is a proof-of-concept targeting simple source and target languages, we nevertheless applied it to produce a verified assembly-level model of an input-handling system for the CDDC (Beaumont et al., 2016), a nontrivial mixed-sensitivity concurrent program.

We expect this decomposition principle to remain applicable in reducing noninterferencerefinement proof efforts for compilers that overcome the specific limitations of ours here. For example, a compiler that inserts padding to equalise the time taken on either side of a High-branch — which may change when it expands expressions into multiple instructionsmay instantiate the decomposition principle with a more sophisticated concrete coupling invariant that does not require pc-security.

This work serves to demonstrate that verified security-preserving compilation for mixedsensitivity concurrent programs is now within reach, by augmenting traditional proof obligations for verified compilation (e.g. square-shaped semantics preservation) with those specific to security (e.g. absence of termination- and timing-leaks) as depicted in Figure 4. We hope that this work paves the way for future large-scale verified security-preserving compilation efforts.

\section{Acknowledgements}

We would like to thank our anonymous referees, and to thank again all those who provided feedback on the conference version of this paper (Sison \& Murray, 2019) and on Robert Sison's PhD thesis (Sison, 2020). This paper describes research that was conducted during Robert's PhD candidature at UNSW Sydney and CSIRO's Data61, which was funded by an Australian Government Research Training Program (RTP) Scholarship and a CSIRO Data61 Research Project Award. We thank the Trustworthy Systems group at CSIRO's Data61 for cultivating an excellent working and learning environment.

\section{Conflicts of Interest}

None 


\section{References}

Abate, C., Blanco, R., Garg, D., Hritcu, C., Patrignani, M. and Thibault, J. (2019) Journey beyond full abstraction: Exploring robust property preservation for secure compilation. 32nd IEEE Computer Security Foundations Symposium, CSF 2019, Hoboken, NJ, USA, June 25-28, 2019 pp. 256-271. IEEE.

Almeida, J. B., Barbosa, M., Barthe, G., Blot, A., Grégoire, B., Laporte, V., Oliveira, T., Pacheco, H., Schmidt, B. and Strub, P.-Y. (2017) Jasmin: High-assurance and highspeed cryptography. Proceedings of the 2017 ACM SIGSAC Conference on Computer and Communications Security. CCS '17, pp. 1807-1823. ACM.

Barthe, G., Basu, A. and Rezk, T. (2004) Security types preserving compilation: (extended abstract). Steffen, B. and Levi, G. (eds), Verification, Model Checking, and Abstract Interpretation, 5th International Conference, VMCAI 2004, Venice, Italy, January 11-13, 2004, Proceedings. Lecture Notes in Computer Science 2937, pp. 2-15. Springer.

Barthe, G., Rezk, T., Russo, A. and Sabelfeld, A. (2007a) Security of multithreaded programs by compilation. Biskup, J. and López, J. (eds), Computer Security - ESORICS 2007, 12th European Symposium On Research In Computer Security, Dresden, Germany, September 24-26, 2007, Proceedings. Lecture Notes in Computer Science 4734, pp. 2 18. Springer.

Barthe, G., Rezk, T. and Basu, A. (2007b) Security types preserving compilation. Comput. Lang. Syst. Struct. 33(2):35-59.

Barthe, G., Rezk, T., Russo, A. and Sabelfeld, A. (2010) Security of multithreaded programs by compilation. ACM Trans. Inf. Syst. Secur. 13(3):21:1-21:32.

Barthe, G., Grégoire, B. and Laporte, V. (2018) Secure compilation of side-channel countermeasures: The case of cryptographic "constant-time". 31st IEEE Computer Security Foundations Symposium, CSF 2018, Oxford, United Kingdom, July 9-12, 2018 pp. 328343. IEEE Computer Society.

Barthe, G., Blazy, S., Grégoire, B., Hutin, R., Laporte, V., Pichardie, D. and Trieu, A. (2020) Formal verification of a constant-time preserving $\mathrm{C}$ compiler. Proc. ACM Program. Lang. 4(POPL):7:1-7:30.

Beaumont, M., McCarthy, J. and Murray, T. (2016) The cross domain desktop compositor: Using hardware-based video compositing for a multi-level secure user interface. Schwab, S., Robertson, W. K. and Balzarotti, D. (eds), Proceedings of the 32nd Annual Conference on Computer Security Applications, ACSAC 2016, Los Angeles, CA, USA, December 5-9, 2016 pp. 533-545. ACM.

Cavalcanti, A. and Naumann, D. A. (2002) Forward simulation for data refinement of classes. Eriksson, L.-H. and Lindsay, P. A. (eds), FME 2002:Formal Methods-Getting IT Right pp. 471-490. Springer Berlin Heidelberg.

Clarkson, M. R. and Schneider, F. B. (2010) Hyperproperties. J. Comput. Secur. 18(6): 1157-1210. 
de Roever, W. P. and Engelhardt, K. (1998) Data Refinement: Model-oriented Proof Theories and their Comparison. Cambridge Tracts in Theoretical Computer Science, vol. 46. Cambridge University Press.

Focardi, R., Gorrieri, R. and Panini, V. (1995) The security checker: a semantics-based tool for the verification of security properties. Proceedings The Eighth IEEE Computer Security Foundations Workshop pp. 60-69.

Frumin, D., Krebbers, R. and Birkedal, L. (2021) Compositional non-interference for finegrained concurrent programs. 42nd IEEE Symposium on Security and Privacy (S\&P'21), to appear; CoRR abs/1910.00905.

Jones, C. B. (1981) Development Methods for Computer Programs including a Notion of Interference. D.Phil. thesis, University of Oxford.

Kaufmann, T., Pelletier, H., Vaudenay, S. and Villegas, K. (2016) When constant-time source yields variable-time binary: Exploiting curve25519-donna built with msvc 2015. Cryptology and Network Security pp. 573-582. Springer International Publishing.

Klein, G., Andronick, J., Elphinstone, K., Murray, T., Sewell, T., Kolanski, R. and Heiser, G. (2014) Comprehensive formal verification of an OS microkernel. ACM Transactions on Computer Systems 32(1):2:1-2:70.

Kumar, R., Myreen, M., Norrish, M. and Owens, S. (2014) CakeML: A verified implementation of ML. Peter Sewell (ed), ACM SIGPLAN-SIGACT Symposium on Principles of Programming Languages pp. 179-191. ACM Press.

Leroy, X. (2009) A formally verified compiler back-end. J. Autom. Reason. 43(4):363-446.

Lochbihler, A. (2018) Mechanising a type-safe model of multithreaded java with a verified compiler. Journal of Automated Reasoning 61(1):243-332.

Lynch, N. and Vaandrager, F. (1996) Forward and backward simulations. Inf. Comput. 128(1):1-25.

Mantel, H., Sands, D. and Sudbrock, H. (2011) Assumptions and guarantees for compositional noninterference. IEEE Computer Security Foundations Symposium pp. 218-232. IEEE.

Mantel, H., Müller-Olm, M., Perner, M. and Wenner, A. (2015) Using dynamic pushdown networks to automate a modular information-flow analysis. 25th International Symposium on Logic Based Program Synthesis and Transformation (LOPSTR).

Masticola, S. P. and Ryder, B. G. (1993) Non-concurrency analysis. Proceedings of the Fourth ACM SIGPLAN Symposium on Principles and Practice of Parallel Programming. PPOPP '93, pp. 129-138. ACM.

Molnar, D., Piotrowski, M., Schultz, D. and Wagner, D. (2006) The program counter security model: Automatic detection and removal of control-flow side channel attacks. Proceedings of the 8th International Conference on Information Security and Cryptology. ICISC'05, pp. 156-168. Springer-Verlag. 
Murray, T. (2015) On high-assurance information-flow-secure programming languages. ACM SIGPLAN Workshop on Programming Languages and Analysis for Security pp. 4348.

Murray, T., Sison, R., Pierzchalski, E. and Rizkallah, C. (2016a) Compositional security-preserving refinement for concurrent imperative programs. Archive of Formal Proofs June. http://isa-afp.org/entries/Dependent_SIFUM_Refinement. shtml, Formal proof development.

Murray, T., Sison, R., Pierzchalski, E. and Rizkallah, C. (2016b) Compositional verification and refinement of concurrent value-dependent noninterference. IEEE 29th Computer Security Foundations Symposium, CSF 2016, Lisbon, Portugal, June 27 - July 1, 2016 pp. 417-431. IEEE Computer Society.

Murray, T., Sison, R., Pierzchalski, E. and Rizkallah, C. (2016c) A dependent security type system for concurrent imperative programs. Archive of Formal Proofs June. http:// isa-afp.org/entries/Dependent_SIFUM_Type_Systems.html, Formal proof development.

Murray, T., Sison, R. and Engelhardt, K. (2018) Covern: A logic for compositional verification of information flow control. European Symposium on Security and Privacy pp. 16-30. IEEE.

Patrignani, M. and Garg, D. (2017) Secure Compilation and Hyperproperty Preservation. IEEE 30th Computer Security Foundations Symposium, CSF 2017, Santa Barbara, USA, August 21 - 25, 2017. CSF'17.

Patrignani, M. and Garg, D. (2019) Robustly safe compilation. Programming Languages and Systems pp. 469-498. Springer International Publishing.

Patrignani, M., Ahmed, A. and Clarke, D. (2019) Formal approaches to secure compilation: A survey of fully abstract compilation and related work. ACM Comput. Surv. 51(6):125:1-125:36.

Podkopaev, A., Lahav, O. and Vafeiadis, V. (2019) Bridging the gap between programming languages and hardware weak memory models. Proc. ACM Program. Lang. 3(POPL):69:1-69:31.

Sabelfeld, A. and Sands, D. (2000) Probabilistic noninterference for multi-threaded programs. Proceedings of the 13th IEEE Workshop on Computer Security Foundations. CSFW '00, pp. 200-. IEEE Computer Society.

Sison, R. (2020) Proving Confidentiality and Its Preservation Under Compilation for Mixed-Sensitivity Concurrent Programs. PhD thesis, University of New South Wales, Sydney. http://doi.org/10.26190/5fab5c0a76454.

Sison, R. and Murray, T. (2019) Verifying That a Compiler Preserves Concurrent ValueDependent Information-Flow Security. Harrison, J., O'Leary, J. and Tolmach, A. (eds), 10th International Conference on Interactive Theorem Proving (ITP 2019). Leibniz International Proceedings in Informatics (LIPIcs) 141, pp. 27:1-27:19. Schloss DagstuhlLeibniz-Zentrum fuer Informatik. 
Staples, M., Jeffery, R., Andronick, J., Murray, T., Klein, G. and Kolanski, R. (2014) Productivity for proof engineering. Empirical Software Engineering and Measurement p. 15.

Tedesco, F. D., Sands, D. and Russo, A. (2016) Fault-resilient non-interference. IEEE 29th Computer Security Foundations Symposium, CSF 2016, Lisbon, Portugal, June 27 - July 1, 2016 pp. 401-416. IEEE Computer Society.

Terauchi, T. and Aiken, A. (2005) Secure information flow as a safety problem. Hankin, C. and Siveroni, I. (eds), Static Analysis pp. 352-367. Springer Berlin Heidelberg.

Volpano, D. and Smith, G. (1998) Probabilistic noninterference in a concurrent language. Proceedings. 11th IEEE Computer Security Foundations Workshop (Cat. No.98TB100238) pp. 34-43.

\section{A Label allocation and sequential composability}

The wr-compiler fixes the label type $L a b \triangleq n a t$ to allow it to ensure freshness merely by using the highest natural number reached so far on a "next label" counter (the argument $n l$ in Example 5.1; it then increments the counter before passing it to subsequent calls, and outputs the next available unused label on return (the return value $n l^{\prime}$ in the example).

Relative to this scheme, we prove that two consecutively compiled RISC programs-in the sense that the relevant outputs from the first call are fed directly into the second callonly ever jump to locations within themselves (and not in the other).

Specifically, we define two RISC programs $P_{1}, P_{2}$ to be joinable if they are both:

- joinable-forward: $P_{1}$ only ever jumps to labels that are either

- labelling an instruction in $P_{1}$ itself, or

- the label of the very first instruction in $P_{2}$.

- joinable-backward: $P_{2}$ does not jump to any of the labels of instructions in $P_{1}$.

The lemma we prove then says that two RISC programs output by consecutive invocations of the wr-compiler are joinable.

Proving that the control flow of programs compiled by the wr-compiler always remains self-contained in this manner facilitates reasoning about their sequential composition.

\section{B Register allocation scheme model}

Like Tedesco et al. (2016) we generalise over the (user-supplied) register allocation scheme, and assume there are enough registers to service the maximum depth of expressions in the source program. We leave for future work the modelling and analysis of a compiler phase that spills register contents to memory, in order to make this assumption unnecessary.

Here we model the (user-supplied) register allocation scheme with two functions reg_alloc and reg_alloc_cached on the register record $\Phi$ (see Section 5.1) and the set $A$ 
of registers whose contents are needed to evaluate the current expression. To avoid loading from memory unnecessarily, the compiler may first call reg_alloc_cached $\Phi A v$ to identify a register that $\Phi$ records as already containing the variable $v$. When the compiler needs a fresh register, it will call reg_alloc $\Phi A$. Neither function is allowed to allocate a register in $A$, so the allocator is permitted to fail if it cannot find any suitable register. However, registers typically become available again as expression evaluation is resolved.

\section{Informal descriptions of cases of refinement relation $\mathscr{R}_{\mathrm{wr}}$}

\section{C.1 Base cases}

- stop: This case relates a terminated While program with a terminated RISC program (i.e. one where the program counter is at the length of the program text).

- skip_nop: This case relates the While program skip with the configuration where the program counter is at the start of the RISC program [Nop].

- assign_expr: This case relates the expression evaluation part (for the expression $e$ ) of the While program $v:=e$ with the corresponding part of the RISC program obtained by compiling it with the wr-compiler.

- assign_store: As for assign_expr, but for the very last Store instruction that commits the result of the expression evaluation back to shared memory variable $v$.

It asserts additionally that $v$ must be stable if lock-governed, and non-lock-governed otherwise. This prevents threads from violating the locking discipline (see Section 3.1).

- lock_acq: This case relates $\operatorname{lock}(k)$ with LockAcq $k$.

- lock_rel: This case relates $\operatorname{unlock}(k)$ with LockRel $k$.

\section{C.2 Inductive cases}

- seq: This case relates the While program $c_{1} ; c_{2}$ with the concatenation $P_{1} @ P_{2}$ of the RISC programs $P_{1}$ and $P_{2}$ that are respectively the outputs of successful consecutive compilation (see Appendix A of $c_{1}$ and $c_{2}$ by the wr-compiler. It is intended for cases where the While (resp. RISC) program is currently in $c_{1}$ (resp. $P_{1}$ ).

It is an inductive case of $\mathscr{R}_{\mathrm{wr}}$, in that:

- $c_{1}$ is required to be related by $\mathscr{R}_{\mathrm{wr}}$ to the present location in $P_{1}$.

- For all local configurations that obey the compiled-cmd-config-consistent requirements, $c_{2}$ is required to be related by $\mathscr{R}_{\mathrm{wr}}$ to the first instruction of $P_{2}$. This quantification ensures that $\mathscr{R}_{\mathrm{wr}}$ remains closed when execution progresses from the first program to the second program.

It asserts that $P_{1}$ and $P_{2}$ are joinable (Appendix A), which is particularly relevant here to ensure that $P_{1}$ can only jump to locations within or at the end of itself (i.e. the start of $P_{2}$ ). 
- join: This case relates a While program $c$ with an offset $p c>$ length $P_{1}$ into a RISC program $P_{1} @ P_{2}$, assuming the inductive hypothesis that $c$ is related by $\mathscr{R}_{\mathrm{wr}}$ with the offset $p c$ - length $P_{1}$ into the RISC program $P_{2}$ alone.

It is intended primarily for cases where the While (resp. RISC) program is currently in the $c_{2}$ (resp. $P_{2}$ ) of some consecutively compiled $c_{1} ; c_{2}$ (resp. $P_{1}$ concatenated with $P_{2}$ ) but applies more broadly to allow any prepend of dead, unreachable instructions onto the front of a RISC program without breaking $\mathscr{R}_{\mathrm{wr}}$.

It also asserts that $P_{1}$ and $P_{2}$ are joinable, which is important here to ensure that $P_{2}$ cannot jump back into $P_{1}$.

- if_expr: This case relates the expression evaluation part (for the expression $e$ ) of the While program if $e$ then $c_{1}$ else $c_{2}$ fi with the corresponding part (including the conditional jump $\mathbf{J z}$ at the end of expression evaluation) of the RISC program obtained by compiling it with the wr-compiler.

It relies on both $c_{1}$ and $c_{2}$ being related by $\mathscr{R}_{\mathrm{wr}}$ to its compiled RISC counterparts when started with initialisation states judged valid by compiled-cmd-config-consistent.

This case is depicted in full in Figure 10, on page 63, for comparison, Figure 11 depicts the relevant part of the compile-cmd implementation.

- if_c1: This case relates some While program $c_{1}^{\prime}$ reachable from $c_{1}$ with the corresponding part within the $c_{1}$ part of the RISC program obtained by compiling if $e$ then $c_{1}$ else $c_{2}$ fi with the wr-compiler.

It relies on $c_{1}$ being related by $\mathscr{R}_{\mathrm{wr}}$ to its compiled RISC counterpart at the appropriate program counter offset.

- if_c2: As for if_c1, but for $c_{2}$.

- epilogue_step: This case relates a terminated While program to the silent control flow steps navigating to the end of a RISC program from the end of the "then" and "else" branches of a compiled if-conditional.

It works only for the "epilogue" step forms: Jmp and Nop (see Section 5.3.2.

It is inductive in that it asserts closedness of $\mathscr{R}_{\mathrm{wr}}$ over pairwise reachability from the pair currently under consideration-the only case to do so directly.

- while_expr: This case relates the While program (while $e$ do $c$ od)'s initial intermediate step to if $e$ then $(c$; while $e$ do $c$ od) else stop fi, and its expression evaluation part, with the expression evaluation and conditional jump of the RISC program that while $e$ do $c$ od was compiled to by compile-cmd.

It relies on $c$ being related by $\mathscr{R}_{\mathrm{wr}}$ to its compiled RISC counterpart when started with initialisation states judged valid by compiled-cmd-config-consistent.

- while_inner: This case relates some program $c_{I}$; while $e$ do $c$ od reachable from $c$; while $e$ do $c$ od to the loop body part of the RISC program compiled from while $e$ do $c$ od. 
It relies on $c_{I}$ being related by $\mathscr{R}_{\mathrm{wr}}$ to its compiled RISC counterpart at the appropriate program counter offset.

It also carries around the same reliance on $c$ being related by $\mathscr{R}_{\mathrm{wr}}$ to its compiled RISC counterpart for all initialisation states judged valid by compiled-cmd-config-consistent.

- while_loop: This case handles epilogue steps for the inner loop body program, and the final jump back to the beginning of the While-loop.

It requires $\mathscr{R}_{\mathrm{wr}}$ to relate the terminated While program to the end of the compiled loop body, and furthermore also carries around the same reliance on $c$ being related by $\mathscr{R}_{\mathrm{wr}}$ to its compiled RISC counterpart for all initialisation states judged valid by compiled-cmd-config-consistent.

$$
\begin{aligned}
& c=\text { if } e \text { then } c_{1} \text { else } c_{2} \text { fi } \quad \text { compile-cmd-input-reqs } C l n l c \\
& \left(P C s, l^{\prime}, n l_{2}, C^{\prime}, \text { False }\right)=\text { compile-cmd } C l n l c \quad\left(P_{e}, r, C_{1}, \text { False }\right)=\text { compile-expr } C \varnothing l e \\
& \left(P_{1}, l_{1}, n l_{1}, C_{2}, \text { False }\right)=\text { compile-cmd } C_{1} \text { None }(\text { Suc }(\text { Suc } n l)) c_{1} \quad p c \leq \text { length } P_{e} \\
& \left.\left(P_{2}, l_{2}, n l_{2}, C_{3}, \text { False }\right)=\text { compile-cmd } C_{1} \text { (Some } n l\right) n l_{1} c_{2} \quad C_{p c}=(\text { map snd } P C s)[p c] \\
& \text { compiled-cmd-config-consistent } C_{p c} \text { regs } \mathrm{mds} \text { mem regrec-stable } C_{p c} \\
& \forall m d s^{\prime} \text { mem }^{\prime} \text { regs }^{\prime} \text {. compiled-cmd-config-consistent } C_{1} \text { regs }^{\prime} m d s^{\prime} \text { mem }^{\prime} \wedge \text { regrec-stable } C_{1} \\
& \longrightarrow\left(\left(\left\langle c_{1}, \text { mds }^{\prime}, \text { mem }^{\prime}\right\rangle_{\mathrm{w}},\left\langle\left(\left(0, \text { map fst } P_{1}\right), \text { regs }^{\prime}\right), \text { mds }^{\prime}, \text { mem }^{\prime}\right\rangle_{\mathrm{r}}\right) \in \mathscr{R}_{\mathrm{wr}} \wedge\right. \\
& \left.\left(\left\langle c_{2}, \text { mds }^{\prime}, \text { mem }^{\prime}\right\rangle_{\mathrm{w}},\left\langle\left(\left(0, \text { map fst } P_{2}\right), \text { regs }^{\prime}\right), \text { mds }^{\prime}, \text { mem }^{\prime}\right\rangle_{\mathrm{r}}\right) \in \mathscr{R}_{\mathrm{wr}}\right)
\end{aligned}
$$

$$
\left(\langle c, m d s, m e m\rangle_{\mathrm{w}},\langle((p c, \text { map fst } P C s), \text { regs }), m d s, m e m\rangle_{\mathrm{r}}\right) \in \mathscr{R}_{\mathrm{wr}}
$$

Figure 10: Introduction rule for case if_expr of refinement relation $\mathscr{R}_{\mathrm{wr}}$.

This case pertains to the expression-evaluation part of an if-conditional compiled by compile-cmd (see Figure 11). Variables ignored are in gray.

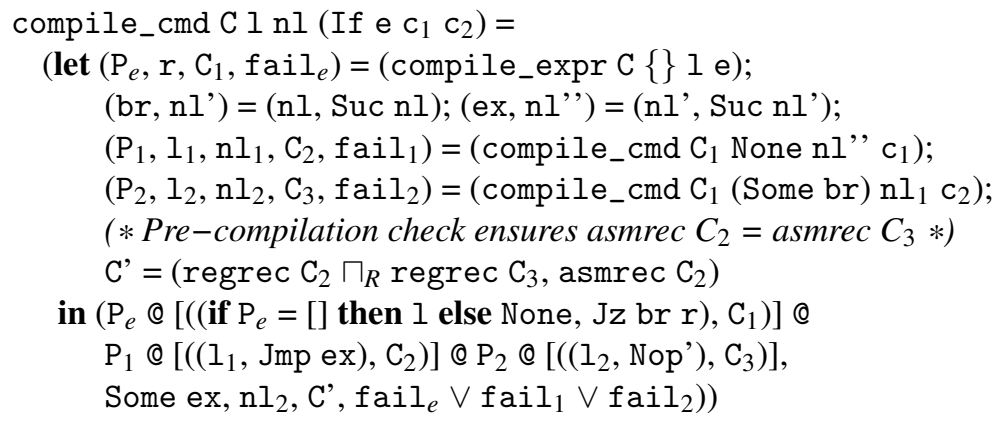

Figure 11: Excerpt of wr-compiler implementation: case for if-conditionals.

This case of the Isabelle/HOL function compile-cmd compiles the While command if $e$ then $c_{1}$ else $c_{2}$ fi. Here, @ denotes concatenation between two RISC program texts, and $\Phi \sqcap_{R} \Phi^{\prime}$ denotes the subset of mappings on which the register records $\Phi$ and $\Phi^{\prime}$ agree. 